\author{
Universidade de São Paulo \\ Instituto de Física
}

\title{
Influência da Formação Estelar versus Buracos Negros de Nucleos Ativos de Galáxias (AGN) na Evolução de Ventos Galácticos
}

\author{
William Eduardo Clavijo Bohórquez
}

Supervisor: Prof. Dra.

ELISABETE MARIA DE GOUVEIA DAL PINO

Dissertação de mestrado apresentada ao Instituto de Física da Universidade de São Paulo, como requisito parcial para a obtenção do título de Mestre em Ciências.

Banca Examinadora:

Prof. Dra. Elisabete Maria de Gouveia Dal Pino (IAG/USP)

Prof. Dr. Diego Falceta Gonçalves (EACH/USP)

Prof. Dr. Eduardo Telles (ON) 
FICHA CATALOGRÁFICA

Preparada pelo Serviço de Biblioteca e Informação do Instituto de Física da Universidade de São Paulo

\section{Clavijo Bohórquez, William Eduardo}

Influência da formação estelar versus buracos negros de núcleos ativos de galáxias (AGN) na evolução de ventos galácticos. São Paulo, 2018.

Dissertação (Mestrado) - Universidade de São Paulo. Instituto de Física. Depto. de Física Matemática.

Orientador: Profa. Dra. Elisabete Maria de Gouveia Dal Pino.

Área de Concentração: Astronomia.

Unitermos: 1. Formação de estrelas; 2. Galáxias ativas; 3. Buracos negros supermassivos; 4 . Ventos galácticos; 5 . Simulações magnetohidrodinâmica. 


\title{
University of São Paulo \\ Physics Institute
}

\section{Star Formation versus Active Galactic Nuclei (AGN) Black Hole feedback in the Evolution of Galaxy Outflows}

\author{
William Eduardo Clavijo Bohórquez
}

Supervisor: Prof. Dra.

ELISABETE MARIA DE GOUVEIA DAL PINO

Dissertation submitted to the Physics Institute of the University of São Paulo in partial fulfillment of the requirements for the degree of Master of Science.

Examining Committee:

Prof. Dra. Elisabete Maria de Gouveia Dal Pino (IAG/USP)

Prof. Dr. Diego Falceta Gonçalves (EACH/USP)

Prof. Dr. Eduardo Telles (ON) 

To my dear María Inés and Aura María 



\section{Agradecimentos/Acknowledgments}

Agradeço ao meu Pai pelos presentes que ele me dá todos os dias. Muita gratidão para minha mãe e meu pai por serem meus grandes mestres. Para minhas irmãs por sua amizade incondicional. Para minha orientadora Elisabete Maria de Gouveia Dal Pino por quem sinto imenso respeito e admiração e quem foi que me ajudou com uma paciência inconsolável na escrita deste texto e especialmente pela sua preocupação e dedicação com mim formação como astrofísico e como ser humano. Para todas as pessoas na Colômbia com quem eu cresci e com quem aprendi rir, amar e sofrer. Para a galera da Nacho: Dani, Mateo, Juancho, Marce, Anita, Alirio, Nata, a galera do Humbold, a praia, o jaguar e o Freud nas tardes de xadrez, café e discussões políticas, matemáticas, artísticas, musicais e de apreciação da vida. Aos meus tios Leo, Mauro, Nelson e os grandes Marco Aurélio Clavijo e Luis Antonio Bohórquez. À galera da infância, às cumplicidades e as gargalhadas: Victor, Eisen, Juan Pablo, Edwin, Rafa, amigos da quadra de futebol e de basquete. Para todas as pessoas aqui no Brasil com quem virei uma garrafa e com quem compartilhei bons momentos. Para Mayo, Dairon, el 'Peje', Huguini, Cris, Raissa, Luis, Tânia, Lucas, Pankaj, Shaila, Mohamed, Stelinha, Paramita, as boas conversas e a pinga. Para a Senhora Luzia para Eliana e para Mara e os meninos, Zigy e Gus por ter me acompanhado por muito tempo nesta viagem. Para todas as pessoas que tornaram minha estada possível aqui no Brasil. Para Claudio Melioli por sua colaboração científica e por me ajudar com o código Gdunov.

Obrigado à agencia $\mathrm{CNPq}$ pelo suporte financiero.

Total obrigado!

A mi Padre por los regalos que me da todos los dias. A mi madre y a mi padre por ser mis grandes maestros. A mis hermanas por su incondicional amistad. A mi orientadora Elisabete Maria de Gouveia Dal Pino por quien siento un inmenso respeto y admiración y fue quien me ayudo con una inconsolable paciencia en la redacción de este texto y sobre todo en su esmero por mi formación como astrofísico. A toda la gente en Colombia con la que crecí y con la que he aprendido a reir, a amar y a sufrir. Al parche de la nacho: Dani, Mateo, Juancho, Marce, Anita, Alirio, Nata, el parche del Humbold, de la Playa, del Jaguar y del Freud por las tardes de ajedrez, de café y de discusiones políticas, matemáticas, artísticas, musicales y de apreciación de la vida. A mis tiitos Leo, Mauro, Nelson, y a mi viejos Marco Aurelio Clavijo y Luis Antonio Bohórquez. A mis amigos de siempre que fueron los de la infancia, las complicidades y las carcajadas. Victor, Eisen, Juan Pablo, Edwin, Rafa, amigos de la cancha de futbol y la de basket. A toda la gente aca en Brasil con la que me tomé una cerveza y con quienes compartí buenos momentos. A Mayo, Dairon, al 'Peje', Huguini, Cris, Juli, Raissa, a Luis Kadowaki, a Tania, Lucas, Pankaj, Shaila, Mohamed, Stelinha, Paramita, por las buenas conversas y la pinga. A la 
Señora Luzia a Eliana y a Mara y a los chicos, Zigy y Gus por haberme acompañado durante un buen tiempo en este viaje. A todas las personas que hicieron posible mi estancia aqui en Brasil. A Claudio Melioli por su colaboración científica y por ayudarme con el código Godunov.

A la agencia CNPq por su apoyo financiero.

Gracias totales! 


\section{Abstract}

Large-scale broad outflows are a common feature in active galaxies, like Seyfert galaxies. In systems like this, where supermassive black hole (SMBH) active galactic nuclei (AGN) coexist with star-forming (SF) regions it is unclear from the observations if the SMBH AGN or the SF (or both) are driving these outflows. In this work, we have studied how both may influence the evolution of the host galaxy and its outflows, considering Seyfertlike galaxies at kilo-parsec (kpc) scales. For this aim, we have extended previous work developed by Melioli \& de Gouveia Dal Pino (2015), who considered purely hydrodynamical outflows driven by both SF and AGN, but considering for the latter only very narrow (collimated) winds. In order to achieve a better understanding of the feedback of these mechanisms on the galaxy evolution and its outflows, here we have included the effects of AGN winds with a larger opening angle too, since conic-shaped winds can improve the interaction with the interstellar medium of the galaxy and thus push more gas into the outflows. Besides, we have also included the effects of magnetic fields in the flow, since these can potentially help to preserve the structures and speed up the outflows. We have performed three-dimensional magneto-hydrodynamical (MHD) simulations considering equilibrium radiative cooling and the effects of AGN-winds with two different opening angles $\left(0^{\circ}\right.$ and $\left.10^{\circ}\right)$, and thermal pressure to magnetic pressure ratios of $\beta \equiv \infty$, 300 and 30 corresponding to magnetic fields $0,0.76 \mu \mathrm{G}$ and $2.4 \mu \mathrm{G}$, respectively. The results of our simulations show that the winds driven by the products of SF (i.e., by explosions of supernovae, SNe) alone can drive outflows with velocities $\sim 100-1000 \mathrm{~km} \mathrm{~s}^{-1}$, mass outflow rates of the order of $50 \mathrm{M}_{\odot} \mathrm{yr}^{-1}$, densities of $\sim 1-10 \mathrm{~cm}^{-3}$, and temperatures between $10^{4}$ and $10^{5} \mathrm{~K}$, which resemble the properties of warm absorbers (WAs) and are also compatible with the velocities of the observed molecular outflows. However, the obtained densities from the simulations are too small and the temperatures too large to explain the observed values in molecular outflows (which have $\mathrm{n} \sim 150-300 \mathrm{~cm}^{-3}$ and $\mathrm{T}<1000 \mathrm{~K}$ ). Collimated AGN winds alone (without the presence of SF-winds) are also unable to drive these outflows, but they can accelerate structures to very high speeds, of the order of $\sim 10.000 \mathrm{~km} \mathrm{~s}^{-1}$, and temperatures $T>10^{6} \mathrm{~K}$ as observed in ultra-fast outflows (UFOs). The introduction of an AGN wind, particularly with a large opening angle, causes the formation of fountain-like structures. This makes part of the expanding gas (pushed by the SF-wind) to fallback into the galaxy producing a 'positive' feedback on the host galaxy evolution. We have found that these effects are more pronounced in presence of magnetic fields, due to the action of extra magnetic forces by the AGN wind producing enhanced fallback that reduces the mass loss rate in the outflows by factors up to 10 . Furthermore, the presence of a collimated AGN wind $\left(0^{\circ}\right)$ causes a significant removal of mass from the core region in a few $100.000 \mathrm{yr}$, but this is soon replenished by gas inflow from the interstellar medium (ISM) when the SNe explosions fully develop. On 
the other hand, an AGN wind with a large opening angle in presence of magnetic fields is able to remove the nuclear gas entirely within a few $100.000 \mathrm{yr}$ and does not allow for later replenishment. Therefore, it quenches the fueling and mass accretion onto the SMBH. This indicates that the duty cycle of these outflows is around a few $100.000 \mathrm{yr}$, compatible with the time-scales inferred for the observed UFOs and molecular outflows. In summary, models that include AGN winds with a larger opening angle and magnetic fields, lead to much larger average speeds than models with no AGN wind, and also allow for more gas to be accelerated to maximum velocities around $\sim 10^{4} \mathrm{~km}$ $\mathrm{s}^{-1}$ (than models with collimated AGN winds), with densities and temperatures which are compatible with those observed in UFOs. However, the structures with intermediate velocities of several $\sim 100 \mathrm{~km} \mathrm{~s}^{-1}$ and densities up to a few $100 \mathrm{~cm}^{-3}$, that in fact could reproduce the observed molecular outflows, have temperatures which are too large to explain the observed molecular features, which have temperatures $\mathrm{T}<1000 \mathrm{~K}$. Besides, these large opening angle AGN winds in magnetized flows reduce the mass loss rates of the outflows to values smaller than those observed both in molecular outflows and UFOs. In future work, we intend to extend the parametric space here investigated and also include new ingredients in our models, such as non-equilibrium radiative cooling, in order to try to reproduce the features above that were not explained by the current model.

Keywords: Active galaxies -Galactic winds - Magneto-hydrodynamical simulations Super Massive Black Holes -Star Formation 


\section{Resumo}

Ventos (em inglês outflows) de ampla abertura e larga escala são uma característica comum em galáxias ativas, como as galáxias Seyfert. Em sistemas como este, onde buracos negros supermassivos (em inglês super-massive black holes, SMBHs) de núcleos galácticos ativos de galáxias (em inglês active galactic nuclei, AGN) coexistem com regiões de formação estelar (em inglês star forming, SF), não está claro das observações se o AGN SMBH ou o SF (ou ambos) são responsaveis pela indução desses ventos. Neste trabalho, estudamos como ambos podem influenciar a evolução da galáxia hospedeira e seus outflows, considerando galáxias tipo Seyfert nas escalas de kilo-parsec (kpc). Para este objetivo, estendemos o trabalho anterior desenvolvido por Melioli \& de Gouveia Dal Pino (2015), que considerou ventos puramente hidrodinâmicos impulsionados tanto pela SF quanto pelo AGN, mas levando em conta para este último apenas ventos bem estreitos (colimados). A fim de obter uma melhor compreensão da influencia (feedback) desses mecanismos sobre a evolução da galáxia e seus outflows, incluímos também os efeitos de ventos de AGN com maior ângulo de abertura, já que ventos em forma de cone podem melhorar a interação com o meio interestelar da galáxia e assim, empurrar mais gás nos outflows. Além disso, incluímos também os efeitos dos campos magnéticos no vento, já que estes podem, potencialmente, ajudar a preservar as estruturas e acelerar os outflows. Realizamos simulações tridimensionais magneto-hidrodinâmicas (MHD) considerando o resfriamento radiativo em equilíbrio de ionização e os efeitos dos ventos do AGN com dois diferentes ângulos de abertura $\left(0^{\circ}\right.$ e $\left.10^{\circ}\right)$ e razões entre a pressão térmica e a pressão magnética $\beta \equiv \infty,=300$ e 30 , correspondentes a campos magnéticos $0,0,76 \mu \mathrm{G}$ e 2,4 $\mu \mathrm{G}$, respectivamente.

Os resultados de nossas simulações mostram que os ventos impulsionados pelos produtos de SF (isto é, pelas explosões de supernovas, SNe) podem direcionar ventos com velocidades $\sim 100-1000 \mathrm{~km} \mathrm{~s}^{-1}$, taxas de perda de massa da ordem de $50 \mathrm{M}_{\odot} /$ ano, densidades de $\sim 1-10 \mathrm{~cm}^{-3}$ e temperaturas entre $10^{4}$ e $10^{5} \mathrm{~K}$, que se assemelham às propriedades dos denominados absorvedores de calor (em inglês warm absorbers, WAs) e também são compatíveis com as velocidades dos outflows moleculares observadas. No entanto, as densidades obtidas nas simulações são muito pequenas e as temperaturas são muito grandes para explicar os valores observados nos outflows moleculares (que têm n 150-300 $\mathrm{cm}^{-3}$ e $\mathrm{T}<1000 \mathrm{~K}$ ). Ventos colimados de AGN (sem a presença de ventos SF) também são incapazes de conduzir outflows, mas podem acelerar estruturas a velocidades muito altas, da ordem de $\sim 10.000 \mathrm{~km} \mathrm{~s}^{-1}$ e temperaturas $T>10^{6} \mathrm{~K}$, tal como observado em ventos ultra rapidos (em inglês, ultra-fast outflows, UFOs). A introdução do vento de AGN, particularmente com um grande ângulo de abertura, causa a formação de estruturas semelhantes a fontes galácticas. Isso faz com que parte do gás em expansão (que está sendo empurrado pelo vento de SF) retorne para a galáxia, produzindo um feedback 
'positivo' na evolução da galáxia hospedeira. Descobrimos que esses efeitos são mais pronunciados na presença de campos magnéticos, devido à ação de forças magnéticas extras pelo vento AGN, o qual intensifica o efeito de retorno do gás (fallback), e ao mesmo tempo reduz a taxa de perda de massa nos outflows por fatores de até 10.

Além disso, a presença de um vento de AGN colimado $\left(0^{\circ}\right)$ causa uma remoção significativa da massa do núcleo da galáxia em poucos 100.000 anos, mas este é logo reabastecido pelo de gás acretante proveniente do meio interestelar (ISM) à medida que as explosões de SNe se sucedem. Por outro lado, um vento de AGN com um grande ângulo de abertura, em presença de campos magnéticos, remove o gás nuclear inteiramente em alguns 100.000 anos e não permite o reabastecimento posterior pelo ISM. Portanto, extingue a acreção de combustível e de massa no SMBH. Isso indica que o ciclo de trabalho desses outflows é de cerca de alguns 100.000 anos, compatível com as escalas de tempo inferidas para os UFOs e outflows moleculares observados.

Em resumo, os modelos que incluem ventos de AGN com um ângulo de abertura maior e campos magnéticos, levam a velocidades médias muito maiores que os modelos sem vento de AGN, e também permitem que mais gás seja acelerado para velocidades máximas em torno de $\sim 10^{4} \mathrm{~km} \mathrm{~s}^{-1}$, com densidades e temperaturas compatíveis com aquelas observadas em UFOs. No entanto, as estruturas com velocidades intermediárias de vários $\sim 100 \mathrm{~km} \mathrm{~s}^{-1}$ e densidades até uns poucos $100 \mathrm{~cm}^{-3}$, que de fato poderiam reproduzir os outflows moleculares observados, têm temperaturas que são muito grandes para explicar as características observadas nos outflows moleculares, que tem temperaturas T< $1000 \mathrm{~K}$. Além disso, estes ventos de AGN não colimados em presença de campos magnéticos reduzem as taxas de perda de massa dos outflows para valores menores que aqueles observados tanto em outflows moleculares quanto em UFOs. Em trabalhos futuros, pretendemos estender o espaço paramétrico aqui investigado e também incluir novos ingredientes em nossos modelos, como o resfriamento radiativo fora do equilibrio, a fim de tentar reproduzir as características acima que não foram explicadas pelo modelo atual.

Palavras chave: Galáxias ativas-Ventos Galáticos-Simulações Magneto-hidrodinâmicasBuracos negros super masivoss-Formação estelar. 


\section{CONTEnts}

1 Introduction 1

1.1 What are Active Galactic Nuclei (AGN)? . . . . . . . . . . . . . 1

1.1.1 Morphology and espectral characteristics of the AGN . . . . . . . 5

1.2 Galaxy Outflows . . . . . . . . . . . . . . . . . . 7

1.3 ¿How does the AGN transmit the energy? . . . . . . . . . . . . 15

1.3.1 Radiative Mode . . . . . . . . . . . . . . . . 15

1.3.2 Mechanical Mode . . . . . . . . . . . . . . . . . 16

1.3.3 Another mechanical mechanism: Winds induced by supernovae (SNe) 17

1.4 On the importance of Magnetic Fields in the study of galaxy outflows . . . 20

1.5 Models of Outflows and Feedback in the literature . . . . . . . . . . . . 20

1.5.1 ¿What will we do in this work? . . . . . . . . . . . . . . 21

1.6 ¿How is this dissertation organized? . . . . . . . . . . . . . . . 22

2 Numerical Model $\quad 23$

2.1 Numerical Method . . . . . . . . . . . . . . . . . . . . . 23

2.2 Computational Domain and Boundary Conditions . . . . . . . . . . . . . . 24

2.3 Initial Setup . . . . . . . . . . . . . . . . . . 25

3 Results of HD and MHD Numerical Simulations 33

3.1 HD-Models . . . . . . . . . . . . . . . . . . . . . 33

3.1.1 Feedback from SN Ia and SN II sources (HD-SN Ia-SN II) . . . . . 33

3.1.2 HD Model with a collimated AGN-wind and SN Ia and SN II (HDSN Ia-SN II-AGN $\left.0^{\circ}\right)$. . . . . . . . . . . . . . . . . . . . 34 
3.1.3 HD-model with SF wind and AGN wind with $10^{\circ}$ opening angle

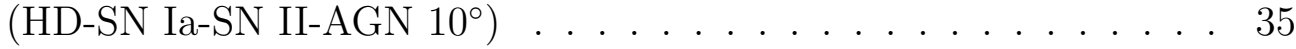

3.2 MHD Models . . . . . . . . . . . . . . . . . 56

3.2.1 MHD Models without AGN-wind (MHD-SN Ia-SN II- $B_{x}-\beta_{300}$ and MHD-SN Ia-SN II- $\left.B_{z}-\beta_{300}\right) \ldots . . . . . .56$

3.2.2 MHD Model including a SF wind, a collimated AGN wind and a horizontal magnetic field (MHD-SN Ia-SN II-AGN $\left.0^{\circ}-B_{x}-\beta_{300}\right)$. . 57

3.2.3 MHD Model including a SF wind, a collimated AGN wind, and a vertical magnetic field (MHD-SN Ia-SN II-AGN $\left.0^{\circ}-B_{z^{-}}-\beta_{300}\right) \quad \ldots$.

3.2.4 MHD Model including a SF wind, an AGN wind with $10^{\circ}$ opening angle, and a horizontal magnetic field (MHD-SN Ia-SN II-AGN 10$\left.B_{x}-\beta_{300}\right) \ldots \ldots \ldots \ldots \ldots \ldots \ldots$

3.2.5 MHD Model including an SF wind, an AGN wind with $10^{\circ}$ opening angle, and a vertical magnetic field (MHD-SN Ia-SN II-AGN $10^{\circ}$ $\left.B_{z}-\beta=300\right) \ldots \ldots \ldots \ldots$. . . . . . . . . . . . . . . . . .

3.2.6 Comparison of MHD models with different initial values of $\beta$. . . . 62

3.3 Discussion and analysis of AGN and SF Feedback . . . . . . . . . . . . 63

3.3.1 Average number density . . . . . . . . . . . . . . . . 64

3.3.2 Volume avergae of $\beta \ldots \ldots \ldots$. . . . . . . . . . . . 64

3.3.3 Mass Transport . . . . . . . . . . . . . . . . . . 65

3.3.4 Average Velocity . . . . . . . . . . . . . . . . . 69

4 Conclusions and Perspectives $r 9$

4.1 Conclusions . . . . . . . . . . . . . . . . . . . . . . . . . 79

4.2 Perspectives for future work . . . . . . . . . . . . . . 81

$\begin{array}{lll}\text { Appendix A } & \text { The Amun Code } & 83\end{array}$ 


\section{List of FiguRES}

1.1 Schematic model of an AGN showing a central black hole (BH), accretion disc, radio-jet, clouds of ionized gas and a molecular torus surrounding the accretion disk (modelled after Urry and Padovani (1995)). The presence of a relativistic jet implies that the AGN would be radio-loud. The diagram also illustrates the unification scheme. The obscuring torus, and its orientation with respect to the observer, determines the view along the line of sight to the central region. A more-or-less direct view manifests itself in spectral characterstics of a Seyfert 1 (Sey 1) galaxy (also radio-quiet QSOs), whereas the obscured view by the torus corresponds to a Seyfert 1 (Sey 2). Quasars and Seyferts differ in luminosity and distance (redshift); classifications Type 1 and 2 refer to orientation. Very few Type 2 quasars (edge-on) are observed, presumbly owing to the difficulty of observing obscured objects at large distances. The dark dots represent ionized gas. The broad line regions are clouds of gas (BLRG) influenced by the central source. The narrow line regions of ionized gas (NLRG) are relatively farther away and visible when the central source is obscured from the line of sight. The outflowing gas acts as a warm absorber, which heavily attenuates the X-ray continuum radiation from the hot corona surrounding the central source (see more details in the text). . . . . . . . . . . . . . . 4 
1.2 Spectral energy distribution of active galactic nuclei. The solid line represents the average espectral energy distribution for radio-loud quasars, and the dashed line refers to radio-quiet quasars. The dotted line is the average espectral energy distribution of 'red AGN' from the $2 \mu \mathrm{m}$ survey called 2MASS (Kuraszkiewicz et al. (2003)), which refers to AGN where the central source is obscured (modelled after M. Elvis et al. (1994), courtesy: J. Kuraszkiewicz and B. Wilkes (2003). Note that $1 \mathrm{eV}=2.42 \times 10^{14} \mathrm{~Hz}$. . .

1.3 M82 is a Starburst Galaxy. The wind generated by SN-driven shocks in its center generates the outflow seen above and below the disc. While the disk is seen in the optical (green and yellow), emitted by starlight, the bipolar cone is composed of hot gases, which emit on X-ray (blue), and is superposed with cold structures emitting in infrared (red) (Geraissate, 2010).

1.4 The Circinus Galaxy lies 13 million light-years away in the southern constellation Circinus. This galaxy is designated a type 2 Seyfert, a class of mostly spiral galaxies that have compact centers and are believed to contain massive black holes. The black hole and its accretion disk are expelling gas out of the galaxy's disk and into its halo (the region above and below the disk). The detailed structure of this gas is seen as magenta-colored streamers extending towards the top of the image. In the center of the galaxy and within the inner starburst ring is a V-shaped structure of gas. The structure appears whitish-pink in this composite image, made up of four filters. Two filters capture the narrow lines from atomic transitions in oxygen and hydrogen; two wider filters detect green and near-infrared light. In the narrow-band filters, the $\mathrm{V}$-shaped structure is very pronounced. This region, which is the projection of a three-dimensional cone extending from the nucleus to the galaxy's halo contains gas that has been heated by radiation emitted by the accreting black hole. (Maiolino et al. (1998)). Credits, NASA, ESA, and the Hubble Heritage (STScI/AURA)-ESA/Hubble Collaboration. . . . . . . . . . . . . . . . . .

1.5 Distribution of (a) outflow velocities, (b) ionization parameter and column density in UFOs obtained from modeling the individual spectra from extended observations of a sample of AGN sources in XMM-Newton and Suzaku data arquives (Tombesi et al. (2011), Gofford et al. (2013)). The red-lined histogram in (c) refers to lower limits in column density. . . . . . 11 
1.6 Relations between the line-of-sight projected distance $\log r / r_{s}$ (with $r_{s}$ the Schwarzchild radius) and ionization parameter $\log \xi$ (panel a), column density $\log N_{H}$ (panel b), outflow velocity $\log v_{\text {out }}$ (panel c) and mass outflow rate $\log \left(\dot{M}_{\text {out }} / \dot{M}_{E d d}\right)$ (panel d). Red points represent WAs, blue points represent UFOs, and green points non-UFOs. The solid lines indicate the best-fitting linear regression curves (Tombesi et al. (2013)) . . . . . . . . . . 12

1.7 This composite image shows the central region of the spiral galaxy NGC 4151, named the "Eye of Sauron". In the "pupil" of the eye, X-rays (blue) from the Chandra X-ray Observatory are combined with optical data (yellow) showing positively charged hydrogen ("H II") from observations with the 1-meter Jacobus Kapteyn Telescope on La Palma. Two X-ray clumps are present at the terminals of the radio-jet (light-blue) (marked as "interaction"), where the outflow (UFO) appears to encounter dense materials (CO gas lane in the NE and HI clump in the SW). The red around the pupil shows neutral hydrogen detected by radio observations with the NSF's Very Large Array. This neutral hydrogen is part of a structure near the center of NGC 4151 that has been distorted by gravitational interactions with the rest of the galaxy and includes material falling towards the center of the galaxy. The yellow blobs around the red ellipse are regions where star formation has recently occurred (Wang et al. (2010)) . . . . . . . . . . . . . 13

1.8 Radio continuum images of $4 \mathrm{C} 12.50$ illustrating the morphology of the radio jet ( $\mathrm{A}$ and $\mathrm{B}$ at the left). (A) The continuum image derived from VLBI data. The red dot indicates the location of the radio core. (B) Continuum image taken from a previous, higher-spatial resolution study (Lister (2003)). The bent structure of the jet and the terminal hot spot in the southern lobe are clearly seen. This hot spot is characterized by an extremely high ( $60 \%$ ) fractional polarization. $\mathrm{A}$ and $\mathrm{B}$ images are displayed on approximately the same scale. At the middle, is shown the distribution of the HI in two velocity channels showing the location of the two clouds of HI detected in absorption (orange-white) superimposed on the continuum of $4 \mathrm{C} 12.50$ (contours). At right, the integrated HI absorption profile is shown in the middle superimposed on the CO profile [taken from Dasyra and Combes (2012) with the HI from Morganti et al. (2005b) and inverted for comparison]. The northern $\mathrm{HI}$ cloud is at the systemic velocity (Morganti (2004)) whereas the southern one is blueshifted $\sim 1000 \mathrm{~km} \mathrm{~s}^{-1}$ relative to the systemic velocity. The location of the southern unresolved cloud producing the blueshifted HI absorption is cospatial with the bright radio hot spot. A diffuse HI component is also observed. . . . . . . . . . . . 14 
1.9 Very Large Array radio image at $5 \mathrm{GHz}$ of the first identified extragalactic radio source, Cygnus-A (3C 405) (e.g., Krause (2018)). The color scale was adjusted to emphasize the faint jets. Contours reveal the bright hotspots. The total linear extent is $\sim 120 \mathrm{kpc}$. Radio map provided by Carilli et al.

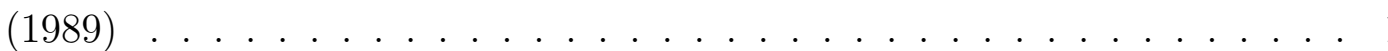

1.10 Rate of energy injected by SNe and lost by radiative cooling in the nuclear region of a Seyfert galaxy considering an active region with cylindrical geometry characterized by a radius $\mathrm{R}=300 \mathrm{pc}$ and a height $\mathrm{h}=200 \mathrm{pc}$ as a function of the SFR. Solid (black) line: energy injected by SNe Ia and SNe II; long-dashed (magenta) line: energy injected by the SNe Ia only; dashed (blue) line: energy lost by radiative cooling assuming a value for the radiative cooling function $\Lambda(T)=10^{-23} \mathrm{erg} \mathrm{s}^{-1} \mathrm{~cm}^{-3}$; dot-dashed (red) line: energy lost by radiative cooling assuming a value for the radiative cooling function $\Lambda(T)=10^{-22} \mathrm{erg} \mathrm{s}^{-1} \mathrm{~cm}^{-3}$. The $\mathrm{SFR}$ is given in $\mathrm{M}_{\odot} \mathrm{yr}^{-1}$ ( $\log 10$ scale), while the energy rates are given in units of $10^{41} \mathrm{erg} \mathrm{s}^{-1}$ (log 10 scale). Extracted from Melioli and de Gouveia Dal Pino (2015) . . . . .

2.1 3D initial logarithmic gas density distribution for the central region of the galaxy within $1 \mathrm{kpc}^{3}$. Density is given in $\mathrm{cm}^{-3}$ and distances are given in pc. 28

$2.23 \mathrm{D}$ initial logarithmic gas temperature and pressure distribution (left and right, respectively) for the central region of the galaxy within $1 \mathrm{kpc}^{3}$. Temperature is given in $K$, pressure in $\mathrm{erg} \mathrm{cm}^{-3}$ and distances are given in

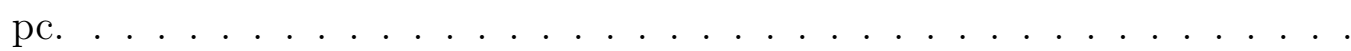

2.3 Edge-on logarithmic gas density distribution (with density given in $\mathrm{cm}^{-3}$ ) for the central region of the galaxy within $1 \mathrm{kpc}^{3}$, with the arrows representing initial configurations of the magnetic field adopted in this work. . On the left $\vec{B}_{z}$ and on the right $\vec{B}_{x}$. Distances are given in pc. . . . . .

3.1 Edge-on logarithmic 2D cuts of the central slice of the model with SFdriven outflow and no AGN-wind, named HD-SN Ia-SN II (See also 2.2). Density is in $\mathrm{cm}^{-3}$ (top), vertical velocity in $\mathrm{km} \mathrm{s}^{-1}$ (top middle), temperature in $\mathrm{K}$ (bottom middle) and pressure in units of $7 \times 10^{-12} \mathrm{erg}$ $\mathrm{cm}^{-3}$ )(bottom) for four different times of evolution: $t=0.225,0.75,3.0$ and 5.0 Myr (from left to right respectively). . . . . . . . . . . .

3.2 Edge-on logarithmic 2D cuts of the central slice of the model with SFdriven outflow and a collimated AGN-wind, named HD-SN Ia-SN IIAGN $0^{\circ}$ (See also 2.2). Density is in $\mathrm{cm}^{-3}$ (top), vertical velocity in $\mathrm{km} \mathrm{s}^{-1}$ (top middle), temperature in $\mathrm{K}$ (bottom middle) and pressure in units of $7 \times 10^{-12} \mathrm{erg} \mathrm{cm}^{-3}$ )(bottom) for four different times of evolution: $t=0.225,0.75,3.0$ and $5.0 \mathrm{Myr}$ (from left to right respectively). . . . . . . 38 
3.3 Edge-on logarithmic 2D cuts of the central slice of the model with SFdriven outflow and a no-collimated AGN-wind (10 opening angle), named HD-SN Ia-SN II-AGN $\mathbf{1 0}^{\circ}$. Density is in $\mathrm{cm}^{-3}$ (top), vertical velocity in $\mathrm{km} \mathrm{s}^{-1}$ (top middle), temperature in $\mathrm{K}$ (bottom middle) and pressure in units of $7 \times 10^{-12} \mathrm{erg} \mathrm{cm}^{-3}$ )(bottom) for four different times of evolution: $t=0.225,0.75,3.0$ and 5.0 Myr (from left to right respectively). . . . . .

3.4 Edge-on logarithmic 2D cuts of the central slice of the model with SF-driven outflow, no AGN-wind and an inital horizontal magnetic field, named MHD-SN Ia-SN II- $B_{x}-\beta_{300}$. Density is in $\mathrm{cm}^{-3}$ (top), vertical velocity in $\mathrm{km} \mathrm{s}^{-1}$ (top middle), temperature in $\mathrm{K}$ (bottom middle) and pressure in units of $7 \times 10^{-12} \mathrm{erg} \mathrm{cm}^{-3}$ )(bottom) for four different times of evolution: $t=0.225,0.75,3.0$ and 5.0 Myr (from left to right respectively).

3.5 Edge-on logarithmic 2D cuts of the central slice of the model with SF-driven outflow, no AGN-wind and an inital horizontal magnetic field, named MHD-SN Ia-SN II- $B_{x}-\beta_{300}$ for four diferent times of evolution: $t=0.225$, 0.75, 3.0 and 5.0 Myr (from left to right respectively). $\beta$ is adimensional (top). $B^{2}$ is in units of $0.76 \mu \mathrm{G}$ (bottom) . . . . . . . . . . .

3.6 Edge-on logarithmic 2D cuts of the central slice of the model with SFdriven outflow, no AGN-wind and an inital vertical magnetic field, named MHD-SN Ia-SN II- $B_{z}-\beta_{300}$. Density is in $\mathrm{cm}^{-3}$ (top), vertical velocity in $\mathrm{km} \mathrm{s}^{-1}$ (top middle), temperature in $\mathrm{K}$ (bottom middle) and pressure in units of $7 \times 10^{-12} \mathrm{erg} \mathrm{cm}^{-3}$ )(bottom) for four different times of evolution: $t=0.225,0.75,3.0$ and 5.0 Myr (from left to right respectively). . . . . . . 42

3.7 Edge-on logarithmic 2D cuts of the central slice of the model with SFdriven outflow, no AGN-wind and an inital vertical magnetic field, named MHD-SN Ia-SN II- $B_{z}-\beta_{300}$ for four diferent times of evolution: $t=0.225$, 0.75, 3.0, 5.0 Myr (from left to right respectively). $\beta$ is adimensional (top). $B^{2}$ is in units of $0.76 \mu \mathrm{G}$ (bottom) . . . . . . . . . . . . . 43

3.8 Edge-on logarithmic 2D cuts of the central slice of the model with SF-driven outflow, a collimated AGN-wind ( $0^{\circ}$ opening angle) and an inital horizontal magnetic field, named MHD-SN Ia-SN II-AGN $0^{\circ}-B_{x}-\beta_{300}$. Density is in $\mathrm{cm}^{-3}$ (top), vertical velocity in $\mathrm{km} \mathrm{s}^{-1}$ (top middle), temperature in $\mathrm{K}$ (bottom middle) and pressure in units of $7 \times 10^{-12} \mathrm{erg} \mathrm{cm}^{-3}$ )(bottom) for four different times of evolution: $t=0.225$, 0.75, 3.0 and 5.0 Myr (from left to right respectively) . . . . . . . . . . . . . . . . . . . 44 
3.9 Edge-on logarithmic 2D cuts of the central slice of the model with SFdriven outflow, collimated AGN-wind ( $0^{\circ}$ opening angle) and an inital horizontal magnetic field, named MHD-SN Ia-SN II-AGN $0^{\circ}-B_{x}-\beta_{300}$ for four diferent times of evolution: $t=0.225,0.75,3.0,5.0 \mathrm{Myr}$ (from left to right respectively). $\beta$ is adimensional (top). $B^{2}$ is in units of $0.76 \mu \mathrm{G}$

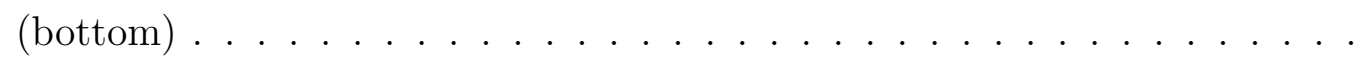

3.10 Edge-on logarithmic 2D cuts of the central slice of the model with SF-driven outflow, a collimated AGN-wind ( $0^{\circ}$ opening angle) and an inital vertical magnetic field, named MHD-SN Ia-SN II-AGN $0^{\circ}-B_{z}-\beta_{300}$. Density is in $\mathrm{cm}^{-3}$ (top), vertical velocity in $\mathrm{km} \mathrm{s}^{-1}$ (top middle), temperature in $\mathrm{K}$ (bottom middle) and pressure in units of $7 \times 10^{-12} \mathrm{erg} \mathrm{cm}^{-3}$ )(bottom) for four different times of evolution: $t=0.225$, 0.75, 3.0, 5.0 Myr (from left to right respectively).

3.11 Edge-on logarithmic 2D cuts of the central slice of the model with SFdriven outflow, collimated AGN-wind ( $0^{\circ}$ opening angle) and an inital vertical magnetic field, named MHD-SN Ia-SN II-AGN $0^{\circ}-B_{z}-\beta_{300}$ for four diferent times of evolution: $t=0.225,0.75,3.0,5.0 \mathrm{Myr}$ (from left to right respectively). $\beta$ is adimensional (top). $B^{2}$ is in units of $0.76 \mu \mathrm{G}$ (bottom) .

3.12 Edge-on logarithmic 2D cuts of the central slice of the model with SFdriven outflow, a no-collimated AGN-wind ( $10^{\circ}$ opening angle) and an inital horizontal magnetic field, named MHD-SN Ia-SN II-AGN $10^{\circ}-B_{x^{-}}$ $\beta_{300}$. Density is in $\mathrm{cm}^{-3}$ (top), vertical velocity in $\mathrm{km} \mathrm{s}^{-1}$ (top middle), temperature in $\mathrm{K}$ (bottom middle) and pressure in units of $7 \times 10^{-12} \mathrm{erg}$ $\mathrm{cm}^{-3}$ )(bottom) for four different times of evolution: $t=0.225,0.75,3.0,5.0$ Myr (from left to right respectively). . . . . . . . . . . . .

3.13 Edge-on logarithmic 2D cuts of the central slice of the model with SFdriven outflow, no-collimated AGN-wind (10 opening angle) and an inital horizontal magnetic field, named MHD-SN Ia-SN II-AGN $10^{\circ}-B_{x}-\beta_{300}$ for four diferent times of evolution: $t=0.225,0.75,3.0,5.0 \mathrm{Myr}$ (from left to right respectively). $\beta$ is adimensional (top). $B^{2}$ is in units of $0.76 \mu \mathrm{G}$

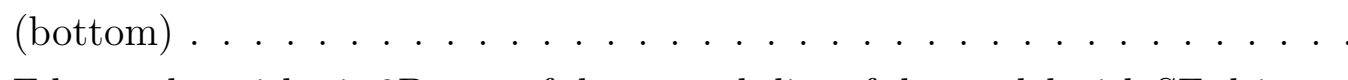

3.14 Edge-on logarithmic 2D cuts of the central slice of the model with SF-driven outflow, a no-collimated AGN-wind (10 opening angle) and an inital vertical magnetic field, named MHD-SN Ia-SN II-AGN $10^{\circ}-B_{z}-\beta_{300}$. Density is in $\mathrm{cm}^{-3}$ (top), vertical velocity in $\mathrm{km} \mathrm{s}^{-1}$ (top middle), temperature in $\mathrm{K}$ (bottom middle) and pressure in units of $\left.7 \times 10^{-12} \mathrm{erg} \mathrm{cm}^{-3}\right)$ (bottom) for four different times of evolution: $t=0.225,0.75,3.0,5.0 \mathrm{Myr}$ (from left to right respectively). . . . . . . . . . . . . . . 50 
3.15 Edge-on logarithmic 2D cuts of the central slice of the model with SFdriven outflow, no-collimated AGN-wind (10 opening angle) and an inital vertical magnetic field, named MHD-SN Ia-SN II-AGN $10^{\circ}-B_{z}-\beta_{300}$ for four diferent times of evolution: $t=0.225,0.75,3.0,5.0 \mathrm{Myr}$ (from left to right respectively). $\beta$ is adimensional (top). $B^{2}$ is in units of $0.76 \mu \mathrm{G}$ (bottom).

3.16 Edge-on logarithmic 2D cuts of the central slice of the model with SF-driven o utflow, a collimated AGN-wind ( $0^{\circ}$ opening angle) and an inital horizontal magnetic field, named MHD-SN Ia-SN II-AGN $0^{\circ}-B_{x}-\beta_{30}$. Density is in $\mathrm{cm}^{-3}$ (top), vertical velocity in $\mathrm{km} \mathrm{s}^{-1}$ (top middle), temperature in $\mathrm{K}$ (bottom middle) and pressure in units of $7 \times 10^{-12} \mathrm{erg} \mathrm{cm}^{-3}$ )(bottom) for four different times of evolution: $t=0.225,0.75,3.0,5.0 \mathrm{Myr}$ (from left to right respectively).

3.17 Edge-on logarithmic 2D cuts of the central slice of the model with SF-driven outflow, collimated AGN-wind ( $0^{\circ}$ opening angle) and an inital horizontal magnetic field, named MHD-SN Ia-SN II-AGN $0^{\circ}-B_{x}-\beta=30$ for four diferent times of evolution: $t=0.225,0.75,3.0,5.0 \mathrm{Myr}$ (from left to right respectively). $\beta$ is adimensional (top). $B^{2}$ is in units of $2.40 \mu \mathrm{G}$ (bottom) .

3.18 Edge-on logarithmic 2D cuts of the central slice of the model with SFdriven outflow, a no-collimated AGN-wind (10 opening angle) and an inital horizontal magnetic field, named MHD-SN Ia-SN II-AGN 10$B_{x}-\beta_{30}$. Density is in $\mathrm{cm}^{-3}$ (top), vertical velocity in $\mathrm{km} \mathrm{s}^{-1}$ (top middle), temperature in $\mathrm{K}$ (bottom middle) and pressure in units of $7 \times 10^{-12} \mathrm{erg}$ $\mathrm{cm}^{-3}$ )(bottom) for four different times of evolution: $t=0.225,0.75,3.0,5.0$ Myr (from left to right respectively). . . . . . . . . . . . .

3.19 Edge-on logarithmic 2D cuts of the central slice of the model with SFdriven outflow, no-collimated AGN-wind ( $10^{\circ}$ opening angle) and an inital horizontal magnetic field, named MHD-SN Ia-SN II-AGN $10^{\circ}-B_{x}-\beta_{30}$ for four diferent times of evolution: $t=0.225,0.75,3.0,5.0 \mathrm{Myr}$ (from left to right respectively). $\beta$ is adimensional (top). $B^{2}$ is in units of $2.40 \mu \mathrm{G}$ (bottom) .

3.20 Detail of clumps at $z \sim 300 \mathrm{pc}$ in the 2D density map of the model MHD-SN Ia-SN II-AGN $0^{\circ}-B_{x^{-}} \beta_{300}$ of Figures 3.8 and 3.9. The yellow arrows stand for the projected magnetic field vectors in the plane. Here, the clumps (red) are more preserved than in the HD model couterpart, because of the

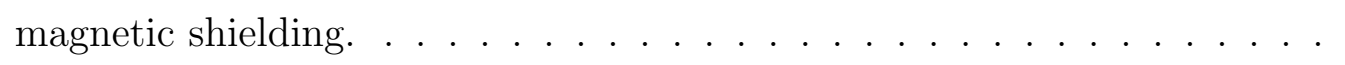

3.21 Detail of the early evolution of the model MHD-SN Ia-SN II-AGN $10^{\circ}$ $B_{x}-\beta_{300}$, at $\mathrm{t}=0.250 \mathrm{Myr}$ when the jet is just crossing the disk to the halo. 
3.22 3D volume density contour plot for the model MHD-SN Ia-SN II-AGN $10^{\circ}$ $B_{x^{-}} \beta_{300}$ at $t=5 \mathrm{Myr}$ showing SN bubbles in the disk and the SF-driven wind that is much more massive than the AGN-wind not seen here because its much smaller density. The magnetic field lines are represented as blue lines. Top bar color gives the density in $\mathrm{cm}^{-3}$ and bottom bar color gives the magnitude of magnetic field $|B|$ in units of $0.76 \mu G \ldots \ldots$. . . .

3.23 Left: 3D helical magnetic field lines warped around the jet. 2D logarithmic slices for density $\left(\mathrm{cm}^{-3}\right)$ and thermal pressure (middle and right respectively). Four recollimation shocks are formed over $\sim 0.2 \mathrm{pc}$ and $\sim 0.4 \mathrm{pc}$ from the central region at the northern and southern jets. As the jet expands laterally, its thermal pressure decreases with distance along the direction of propagation. When the jet pressure reaches the ambient pressure the jet begins to re-collimate. The jet periodically expands and re-collimates, producing a series of biconical or normal shocks along the jet axis (for further detail, see, .e.g., Nawaz et al. (2014)). Four of these shocks appear as knots in the pressure plot at right. Logarithmic scale for density is at left and for pressure is at right. Density is in $\mathrm{cm}^{3}$, pressure in reference units $7 \times 10^{12} \mathrm{erg}^{-3}$, and magnetic field in units of reference, $0.76 \mu$ G. . . . . . . . . . . . . . . . . . .

3.24 Volume averaged number density in the systems as a function of time. Density is in $\mathrm{cm}^{-3}$ and time in Myr. The colors of the curves stand for the models according to the legend. . . . . . . . . . . . . . . .

3.25 Volume averaged value of $\beta$ in the models as a function of time. Time is in Myr. The colors of the curves stand for the models according to the legend. 65

3.26 Total mass within the core region of the galaxy $(r \leq 40 \mathrm{pc})$ as a function of time for all models investigated in this work with inital $\beta \equiv \infty, 300$ and 30 (see Table 2.2). The colors of the curves stand for the models according to the legend. . . . . . . . . . . . . . . . . . . . 66

3.27 Total mass within the disk region of the galaxy $(|z| \leq 200 \mathrm{pc}$,$) as a function$ of time for all models investigated in this work with inital $\beta \equiv \infty, 300$ and 30 (see Table 2.2). The colors of the curves stand for the models according to the legend. . . . . . . . . . . . . . . . . . .

3.28 Time evolution of the gas total mass loss rate from the galaxy thick disk $(|z| \leq 200 \mathrm{pc})$ for all the models. Time is in Myr, and mass-loss rate is in units of $\mathrm{M}_{\odot} \mathrm{yr}^{-1}$. The plot at the bottom left, shows a zoom on the curves for the models MHD-SN Ia-SN II-AGN $10^{\circ}-B_{x^{-}} \beta=300$ (green), MHDSN Ia-SN II-AGN $10^{\circ}-B_{z}-\beta=300$ (brown) and MHD-SN Ia-SN II-AGN $10^{\circ}-B_{x}-\beta=30$ (orange curve) . . . . . . . . . . . . . . 
3.29 Volume average velocity for the models: a. evolution over the first $1.0 \mathrm{Myr}$, and $\mathbf{b}$. evolution over $6 \mathrm{Myr}$. The velocity is calculated by summing the velocities of each cell of the domain and dividing by the total number of cells. . . . . . . . . . . . . . . . . . . .

3.30 Two dimensional histograms of the vertical velocity versus density $(\mathrm{V}-\mathrm{n})$ (top), and velocity vs. temperature (V-T)(bottom) calculated considering every cell within the whole simulated system at $t=0.225 \mathrm{Myr}$, for the three models with SF-wind only, from left to right: the HD model, the model with initial horizontal magnetic field, and the model with initial vertical field, both with $\beta=300$. Velocity is in $\mathrm{km} \mathrm{s}^{-1}$, density in $\mathrm{cm}^{-3}$ and temperature in $\mathrm{K}$, all in logarithmic scale. The color bar indicates the cell number normalized to their total number. The names of the models are indicated at the top of each panel. . . . . . . . . . . . . . . . . . . 71

3.31 Two dimensional histograms of the vertical velocity versus density (V-n) (top), and velocity vs. temperature (V-T)(bottom) calculated considering every cell within the whole simulated system at $t=5 \mathrm{Myr}$, for the three models with SF-wind only, from left to right: the HD model, the model with initial horizontal magnetic field, and the model with initial vertical field, both with $\beta=300$. Velocity is in $\mathrm{km} \mathrm{s}^{-1}$, density in $\mathrm{cm}^{-3}$ and temperature in $\mathrm{K}$, all in logarithmic scale. The color bar indicates the cell number normalized to their total number. The names of the models are indicated at the top of each panel. . . . . . . . . . . . . .

3.32 Two dimensional histograms of the vertical velocity versus density (V-n) (top), and velocity vs. temperature (V-T)(bottom) calculated considering every cell within the whole simulated system at $t=0.225 \mathrm{Myr}$, for the three models with collimated AGN-wind, from left to right: the HD model, the model with initial horizontal magnetic field, and the model with initial vertical field, both with $\beta=300$. Velocity is in $\mathrm{km} \mathrm{s}^{-1}$, density in $\mathrm{cm}^{-3}$ and temperature in $\mathrm{K}$, all in logarithmic scale. The color bar indicates the cell number normalized to their total number. The names of the models are indicated at the top of each panel. . . . . . . . . . . . . . . . . . 
3.33 Two dimensional histograms of the vertical velocity versus density (V-n) (top), and velocity vs. temperature (V-T)(bottom) calculated considering every cell within the whole simulated system at $t=5 \mathrm{Myr}$, for the three models with collimated AGN-wind, from left to right: the HD model, the model with initial horizontal magnetic field, and the model with initial vertical field, both with $\beta=300$. Velocity is in $\mathrm{km} \mathrm{s}^{-1}$, density in $\mathrm{cm}^{-3}$ and temperature in $\mathrm{K}$, all in logarithmic scale. The color bar indicates the cell number normalized to their total number. The names of the models are indicated at the top of each panel. . . . . . . . . . . . . . .

3.34 Two dimensional histograms of the vertical velocity vs. density (V-n)(top) and velocity vs. temperature $(\mathrm{V}-\mathrm{T})$ (bottom)at $t=0.225 \mathrm{Myr}$, for the models with $\mathrm{AGN}$-wind with $10^{\circ}$ opening angle, from left to right: the HD model, the model with initial horizontal magnetic field, and the model with initial vertical field, both with $\beta=300$. Velocity is in $\mathrm{km} \mathrm{s}^{-1}$, density in $\mathrm{cm}^{-3}$ and temperature in $\mathrm{K}$, all in logarithmic scale. The color bar indicates the cell number normalized to their total number. The names of the models are indicated at the top of each panel . . . . . . . . . .

3.35 Two dimensional histograms of the vertical velocity vs. density (V-n)(top) and velocity vs. temperature $(\mathrm{V}-\mathrm{T})$ (bottom)at $t=5 \mathrm{Myr}$, for the models with AGN-wind with $10^{\circ}$ opening angle, from left to right: the HD model, the model with initial horizontal magnetic field, and the model with initial vertical field, both with $\beta=300$. Velocity is in $\mathrm{km} \mathrm{s}^{-1}$, density in $\mathrm{cm}^{-3}$ and temperature in $\mathrm{K}$, all in logarithmic scale. The color bar indicates the cell number normalized to their total number. The names of the models are indicated at the top of each panel . . . . . . . . . . . . .

3.36 Two dimensional histograms of the vertical velocity versus density $(\mathrm{V}-\mathrm{n})$, and versus temperature $(\mathrm{V}-\mathrm{T})$ at $t=5 \mathrm{Myr}$, for the models with AGNwind with $10^{\circ}$ opening angle, nd initial horizontal magnetic field with for two values of $\beta=300$ (left panel), and 30 (right panel). Velocity is in $\mathrm{km}$ $\mathrm{s}^{-1}$, density in $\mathrm{cm}^{-3}$ and temperature in $\mathrm{K}$, all in logarithmic scale. The color bar indicates the cell number normalized to their total number. The names of the models are indicated at the top of each panel. . . . . . . . .

A.1 Centering of volume-averaged conserved variables $U$ and area-averaged components of magnetic field B on the grid. Right: Centering of timeand area-averaged components of the fluxes of $U$ on the grid (picture taken from Stone 2008) . . . . . . . . . . . . . . . . . . . 
A.2 Radiative cooling curve $P_{\text {rad }}$ for opticaly thin gas with radiative looses by: Bremsstrahlung, recombination and emission lines. The radiative cooling rate (eq. A6) is given by $\Lambda=n^{2} P_{\text {rad }}$ erg $\mathrm{cm}^{-3} \mathrm{~s}^{-1}$. The continuous line was calculated by McWhirter et al. (1975) for the solar corona. For comparasion, Pottasch (1965) (chain line) and Cox and Tucker (1969) (dashed line) . . . . . . . . . . . . . . . . . . . 87 


\section{List of TABLES}

1.1 Comparative table for some properties of WAs and UFOs . . . . . . . . . 11

2.1 Parameters for the initial setup of the galaxy model . . . . . . . . . . . 27

2.2 Initial conditions adopted in the HD and MHD Simulations for 11 Different Models . . . . . . . . . . . . . . . . . . 31 
CHAPTER 1

INTRODUCTION

\subsection{What are Active Galactic Nuclei (AGN)?}

The number of stars in a galaxy can vary in a wide range from $10^{7}$ in Dwarf Galaxies to $10^{12}$ as in our Milky Way. Given the luminosity of the Sun as a benchmark $L_{\odot}=3.8 \times 10^{33}$ $\mathrm{erg} \mathrm{s}^{-1}$, the luminosity of a normal galaxy is $L_{G} \sim 10^{11} L_{\odot}$. A large fraction of galaxies in the universe, at least 10\%, are much brighter. To be more specific, the central regions of these galaxies are extremely bright with a central luminosity at least equal to that of the rest of the galaxy. At first sight, one might think that this luminosity can be the result of the light emitted by a big amount of stars accumulated towards the center of the galaxy. However, these ultraluminous central regions exhibit some characteristic observational facts (Pradhan and Nahar (2011))

1. The energy released by the central region ranges from $10^{12}-10^{15} L_{\odot}$, or in other words up to 10.000 times the luminosity of the Milky Way.

2. The region where this energy is released is very compact and occupies a small fraction of a parsec ${ }^{1}$, whereas the galaxy, can extend over tens of kiloparsecs.

3. The spectral energy distribution (SED) is non-Planckian, i.e. it does not follow a black body SED, while the continuum spectrum of the stars does.

4. Ten percent of these bright nuclei are intense radio sources. This radio emission is of non-thermal origin, has a power-law SED and is attributed to Synchrotron

\footnotetext{
${ }^{1} 1 \mathrm{pc} \sim 3 \times 10^{18} \mathrm{~cm}$
} 
radiation (see more below).

5. Variability on short time-scales (from years to some few days) implying that the the source is very compact since, due to causality arguments, the size of the emitting region cannot exceed $\Delta x \sim c \Delta t$, where $c$ is the light speed and $\Delta t$ is the time variability of the radiation.

Something has to drive the enormous activity at the center of these galaxies, also denominated "active galaxies", with a tremendous output of non-stellar form of energy. A particularly interesting and useful fact is that this activity is reflected in an enormous variety of emission and absorption spectra, from neutral hydrogen to highly ionized iron. This gives the means to study the physical conditions of active galaxies. As stressed above, if galaxies were simply a collection of stars, one might expect that their spectra obey a blackbody distribution. And since the total luminosity should be dominated by the brightest and most massive stars, the expected spectra of galaxies should have similar spectral components weighted rather towards the near-ultraviolet and the optical as occurs in the case of most ordinary galaxies, which are not active in the sense described above. For any blackbody (or collection of them as in a large number of stars), the Planck function predicts some output of electromagnetic radiation in all wavelength ranges. However, deviations from this spectral distribution manifest as one examines the central regions of active galaxies and finds that luminosity is non-Planckian in nature. The term generally employed for centers of galaxies with energy output fuelled by some tremendous activity is active galactic nuclei (AGN).

There is a number of sub-classes of objects that fall under the AGN characterization. At one end of extreme luminosities are the quasi-stellar objects, or QSOs, which are identified by their non-zero redshifts or great distances out to $\mathrm{z} \sim 6$. Historically, a number of QSOs was observed by their copious radio emission and called quasars (the acronym for quasi-stellar radio sources). The QSO spectra show significant redshifts of their spectral lines compared the rest frame or laboratory wavelengths. That means that QSOs are at large distances from us and, owing to the cosmological expansion, must have originated at earlier times in the history of the Universe. Quasi-stellar objects may be among the first large-scale objects formed at the earliest epochs. They exhibit an extended emission in radio arising from the nuclear region suggesting the presence of outflows (see more below). Many cataloged quasars are at the most luminous end of AGN luminosity, partly due to selection effects in observing far away objects, which would tend to be the brightest.

Another object in the high luminous tail is the optically violent variable quasar(OVV). This is a type of highly variable quasar. Consists of a few rare, bright radio galaxies, whose visible light output can change by $50 \%$ in a day. 
Other sub-classes of AGNs that are in the low luminosity limit, are the Seyfert galaxies and the radio-galaxies. Seyfert galaxies are active galaxies with a luminosity of $\sim 10^{11} L_{\odot}$ that represent approximately $10 \%$ of galaxies in the universe (Figure 1.4). Seyfert galaxies are normally classified in two types according to observational characteristics. Type 1 Seyferts are generally characterized by the presence of broad emission lines emitted by the so-called broad line regions (BLR). The BLR clouds are moving under the gravitational influence of the nucleus of the galaxy with Doppler broadened emission lines. Observed velocity dispersions of up to $10.000 \mathrm{~km} \mathrm{~s}^{-1}$ or more are seen. By contrast, Seyfert type 2 contain narrow lines. The central source is obscured and only the outer relatively colder regions farther away than the BLR clouds are seen by the observer.

BL Lacs are also low luminous AGNs named after their prototype, BL Lacertae. In contrast to other types of active galactic nuclei, BL Lacs are characterized by rapid and large-amplitude flux variability and significant optical polarization. Because of these properties, the prototype of the class (BL Lac) was originally thought to be a variable star. When compared to the more luminous active nuclei (quasars) with strong emission lines, BL Lac objects have spectra dominated by a relatively featureless non-thermal emission continuum over the entire electromagnetic range. This lack of spectral lines historically hindered BL Lac's identification of their nature and proved to be a hurdle in the determination of their distance (Falomo et al. (2014)).

AGNs are also frequently classified according to their luminosity in radio, as radio-quiet and radio-loud (Wilson and Colbert (1995)). The radio-loud objects produce large-scale radio jets and lobes, with the kinetic power of the jets being a significant fraction of the total bolometric luminosity. These are commonly denominated radio galaxies. On the other hand, the weak radio ejecta of the radio-quiet objects is energetically insignificant. The radio-loud objects are associated with elliptical galaxies which have undergone recent mergers, while the radio-quiets prefer spiral hosts. The space density of the radio-loud at a given optical luminosity is $\sim 10$ times lower than that of the radio-quiet. Despite these differences, the (probably) thermal emissions from the AGN (continua and lines from X-ray to infrared wavelengths) are quite similar in the two classes of object.

Again, something or some process is needed to generate the stupendous amount of energy put out by AGNs. While we do not know yet the precise nature of this central engine of AGN, and maybe even of normal galaxies, the working paradigm that has emerged is a gravitationally accreting supermassive black hole (SMBH), which apparently unifies most of the observed phenomena mentioned above. As we shall see, spectral features of different parts of AGN reveal some physical phenomena, many of which are not presently understood. 


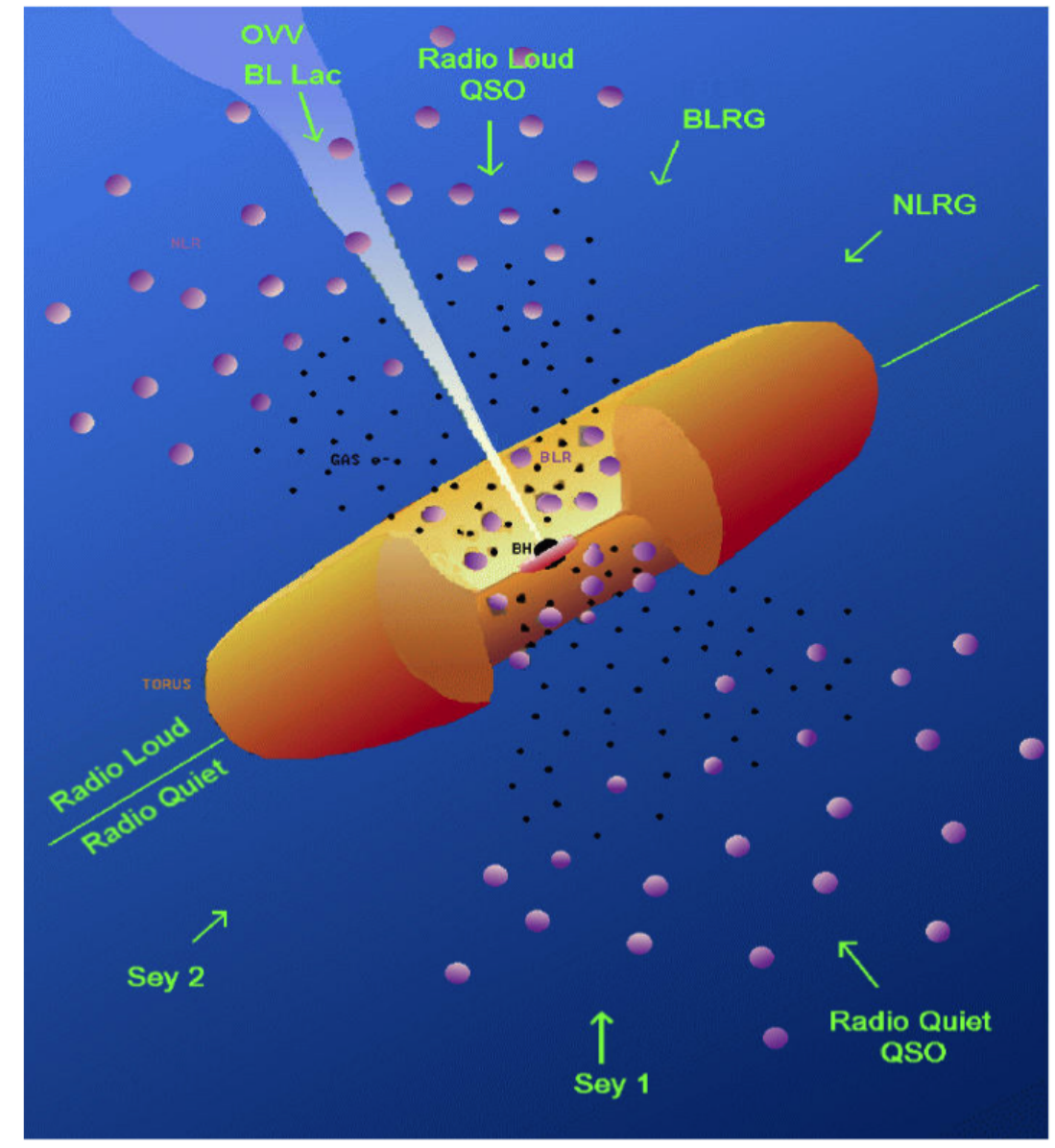

Figure 1.1: Schematic model of an AGN showing a central black hole (BH), accretion disc, radio-jet, clouds of ionized gas and a molecular torus surrounding the accretion disk (modelled after Urry and Padovani (1995)). The presence of a relativistic jet implies that the AGN would be radio-loud. The diagram also illustrates the unification scheme. The obscuring torus, and its orientation with respect to the observer, determines the view along the line of sight to the central region. A more-or-less direct view manifests itself in spectral characterstics of a Seyfert 1 (Sey 1) galaxy (also radio-quiet QSOs), whereas the obscured view by the torus corresponds to a Seyfert 1 (Sey 2). Quasars and Seyferts differ in luminosity and distance (redshift); classifications Type 1 and 2 refer to orientation. Very few Type 2 quasars (edge-on) are observed, presumbly owing to the difficulty of observing obscured objects at large distances. The dark dots represent ionized gas. The broad line regions are clouds of gas (BLRG) influenced by the central source. The narrow line regions of ionized gas (NLRG) are relatively farther away and visible when the central source is obscured from the line of sight. The outflowing gas acts as a warm absorber, which heavily attenuates the X-ray continuum radiation from the hot corona surrounding the central source (see more details in the text). 


\subsubsection{Morphology and espectral characteristics of the AGN}

A basic task in the AGN research is to understand how all the sub-classes described in the the previous section are connected, whether or not their characteristics can be understood in a unified scheme that exhibits the underlying physics. Actually, the general consensus is that the AGN activity is driven by supermassive black holes (SMBH). These central engines are thought to drive the observed energetics and structures. The morphology of an AGN comprises a number of apparently distinct regions, as shown schematically in Figure 1.1. The central SMBH is surrounded by an accretion disk formed by infalling matter (donated by the host galaxy). Rotation and conservation of angular momentum requires the formation of an accretion disc. The accretion disk converts gravitational energy of infalling matter into mechanical energy, heating, and radiation. The emergent radiation shows enhanced UV-radiation together with ionized gas outflows, as evidenced by broad absorption in strong lines of atomic species. The thermal emission from the accretion disk can be modeled as a blackbody, modified according to geometry and opacity effects (Reis et al. (2008)). Figure 1.1 also indicates that the different sub-classes of AGN are believed to be similar objects, but which are viewed at different angles depending on their orientation with respect to the observer at the Earth (the line of sight).

The unifying structure of the AGN is also thought to be governed by the angular momentum of accreting matter. The angular momentum of infalling material is conserved, and then its excess is removed by centrifugally driven winds from accretion discs, which, in turn, give account for the observed outflows and jets (see Fabian (2012), King and Pounds (2015), Netzer (2015) for reviews, and references therein). The accretion disk is not directly visible, but a jet of material moving out at relativistic velocities, arising from the interaction of the $\mathrm{SMBH}$ and the infalling matter, is often visible at radio frequencies out to tens of $\mathrm{kpc}$ or Mpc in some cases. As a result of jet and accretion disk emission, the electromagnetic spectrum of AGN ranges from radio waves to gamma-rays at $\mathrm{TeV}$ energies. While synchrotron radio emission resulting from relativistic electrons accelerated in the magnetic fields would be expected from all AGN, the intensity and extent of observed radio emission determines whether the AGN is classified as radioloud or radio-quiet. At high-energy spectrum tail, the X-ray background continuum is generated by means of three radiative processes:

1. Bremsstrahlung radiation due to electrons accelerating in the proximity of ions;

2. Inverse Compton scattering of low energy photons by electrons, and consequent shift to higher frequencies;

3. Synchrotron radiation due to electrons accelerating in a magnetic field. 
These processes play important role in the formation of AGN X-ray spectrum driven by the central black hole. The origin of very high energy emission at gamma-rays ( $\mathrm{TeV}$ energies) is still controversial, but it is also believed to be due to non-thermal radiation processes generated by particles accelerated at relativistic velocities in shocks or by magnetic reconnection, near the AGN or along the jets (e.g., de Gouveia Dal Pino et al. (2015); Singh et al. (2016)).

The most direct evidence of a relativistic accretion disk around a SMBH is an iron line due to $K \alpha$ transition(s) at about $6.4 \mathrm{keV}$ (Tanaka, 1995). The line is seen to have a broadening towards the red, down to about $5.7 \mathrm{keV}$. This indicates gravitational redshift, lowering of the energy of photons emitted, predicted by the theory of general relativity. The material interactions in the vicinity of the disk also result in a hot highly ionized matter in X-ray spectra. The gas outflow, which shows overall soft X-ray absorption, is referred to as the warm absorber (WA). Both the disk and the WA show emission and absorption lines which can be identified in laboratory samples of atomic species ranging from O VII, Fe XVII in the soft X-ray $(\sim 0.5-2 \mathrm{keV})$ to Fe XXV, Fe XXVI in the hard X-ray (6.6-7.0 keV) (Pounds and King (2013)). Farther away from the central source, at distances of tens of pc, but influenced by the gravity of the $\mathrm{SMBH}$, are the broadline-region (BLR) clouds of relatively less ionized gas. The BLR is characterized by bulk Doppler motions with velocities up to thousands of $\mathrm{km} \mathrm{s}^{-1}$, inferred from the width of emission lines such as the $\mathrm{H} \beta(4860 \AA)$ in the optical or C IV $(1541 \AA)$ in the ultraviolet. The BLR gas shows spectral features of typical nebular stages of ionization temperature, but much larger electron densities of $10^{9-12} \mathrm{~cm}^{-3}$ and much broader emission lines from species such as O III and Fe II. Still farther out are the narrow line region (NLR) clouds, quite similar to ionized gas in H II regions of the interstellar medium of our galaxy. The NLR spectra have typically nebular forbidden lines of low-ionization atomic species, such as $[\mathrm{O} \mathrm{II}]$, [O III], [S II] and [Fe II], but at lower densities than the BLR. The central region of the AGN is shrouded in a large molecular torus rotating around the $\mathrm{SMBH}$, as inferred by Doppler blue- and redshifts of $\mathrm{H}_{2} \mathrm{O}$ masers: the maser action in the water molecule is pumped by AGN activity. For example, a sub-parsec maser disk is observed in the AGN NGC 4258 (Messier M106) in the strong $22 \mathrm{GHz}$ radio line (Modjaz et al. (2005)). The immense variety of radiative and material processes occurring within the AGN makes it obvious that their spectral features should encompass practically the entire electromagnetic spectrum, from gamma and hard X-ray to radio. The global spectral energy distribution is such that it remains large and flat across all wavelength ranges, as measured by the quantity $\nu F_{\nu}$ (or equivalently $\nu L_{\nu}$, where $L_{\nu}$ is the luminosity, and $\mathrm{F}_{\nu}$ is the flux at the given frequency $\nu$ ) in any specified range (Figure 1.2). But actual observations reveal large differences in properties of AGNs. Nevertheless, the current paradigm unifying the AGN phenomenology is determined by the essential geometry of 
a SMBH and an accretion disc. The orientation of the accretion disk relative to the observer (on the Earth) and associated activities determine the morphological, spectral, and temporal (time variability) properties. The unified model rests on the same underlying physical processes for all AGNs.

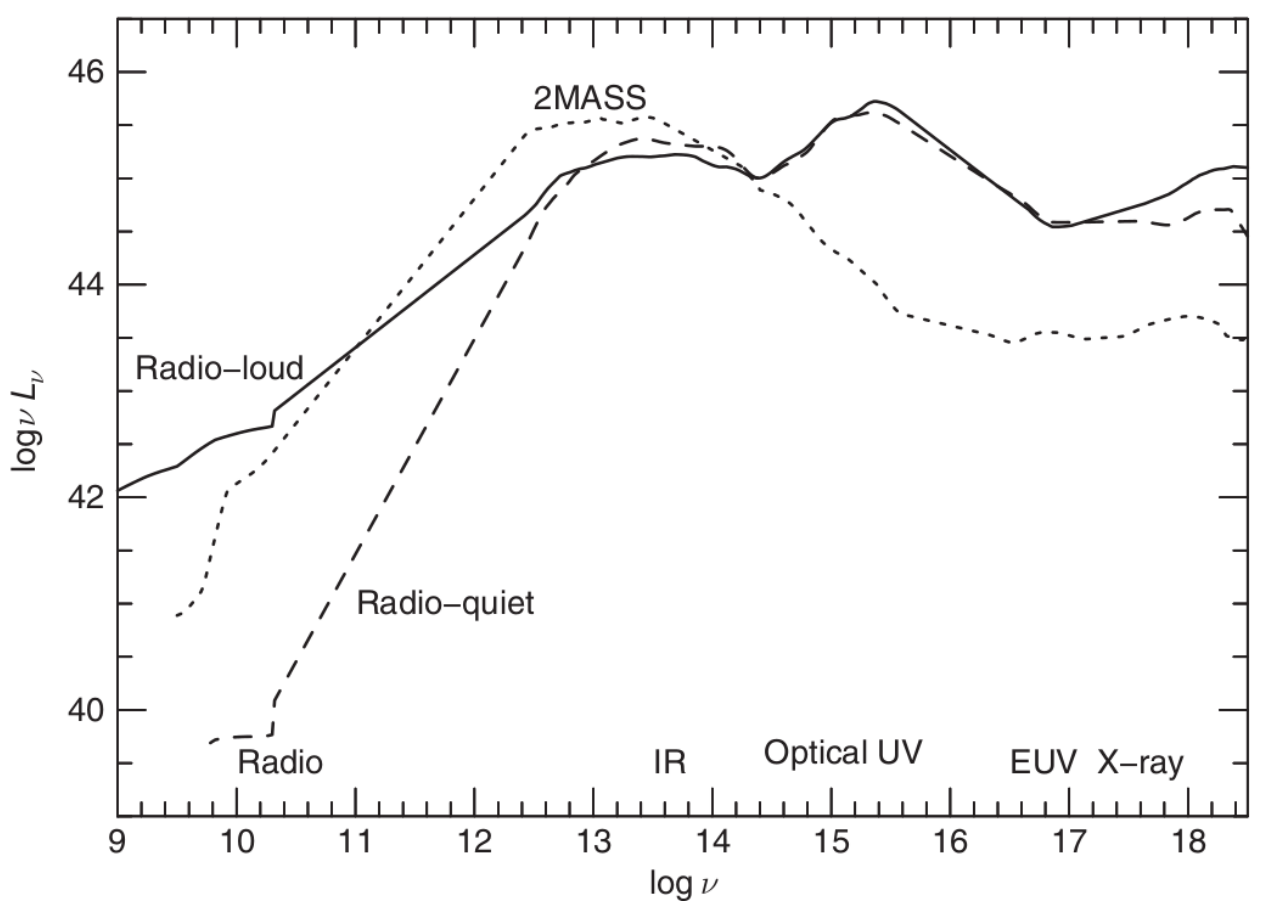

Figure 1.2: Spectral energy distribution of active galactic nuclei. The solid line represents the average espectral energy distribution for radio-loud quasars, and the dashed line refers to radioquiet quasars. The dotted line is the average espectral energy distribution of 'red AGN' from the $2 \mu \mathrm{m}$ survey called 2MASS (Kuraszkiewicz et al. (2003)), which refers to AGN where the central source is obscured (modelled after M. Elvis et al. (1994), courtesy: J. Kuraszkiewicz and B. Wilkes (2003). Note that $1 \mathrm{eV}=2.42 \times 10^{14} \mathrm{~Hz}$.

\subsection{Galaxy Outflows}

Galaxy broad outflows in the last decades have been a very intense field of study in the evolution of galaxies, particularly because they can reveal several physical processes occurring in the nuclear regions of the galaxies from where they are ejected, which otherwise would remain unveiled. Observational evidence shows high-speed outflows extending for a few kiloparsec scales or larger in local galaxies with high star formation rates (SFR) and with active galactic nuclei (AGNs). A large fraction of these outflows are detected in high-redshift sources and make it possible to suspect that outflows may represent a phase in the galactic evolution (see, e.g., Shapley (2003)). Also, the multiphase distribution of the interstellar medium (ISM) of the host galaxy can be affected by such outflows (see, e.g., McKee and Truelove (1995)) and they can influence the dynamical and chemical evolution of the galaxy, as well as enrich with gas metals the intergalactic medium (IGM; 
see, e.g., Heckman et al. (1990); Melioli et al. (2009), Melioli et al. (2013), Melioli et al. (2015), and references therein), and be responsible for the deficit of baryons seen in many galaxies. In addition, outflows may also extinguish star formation (SF) heating up the cold gas and eject it from the host galaxy. High-energy outflows have been proposed as the main cause of the drop in AGN luminosity and the end of the galactic gas fueling onto the central supermassive black hole $(\mathrm{SMBH})$, limiting the active phase of the nucleus to a time-scale of about $10^{8} \mathrm{yr}$.

Most active galaxies exhibit very collimated outflows which are also particularly important for starburst (SB) galaxies, Seyferts and ultra-luminous infrared galaxies (ULIRGs), for which intrinsically large SFR provides the perfect conditions for gas outflow to develop.

Starburst galaxies are systems which exhibit bursts of star formation with rates that can be around 10 times larger than the SFR of normal galaxies like the Milky Way (for which SFR $0.68-1.45 \mathrm{M}_{\odot} \mathrm{yr}^{-1}$; Robitaille and Whitney (2010)). For instance, the starburst prototype M82 has a SFR of the order of $10 \mathrm{M}_{\odot} \mathrm{yr}^{-1}$ (see Figure 1.3 with the starburst galaxy prototype M82).

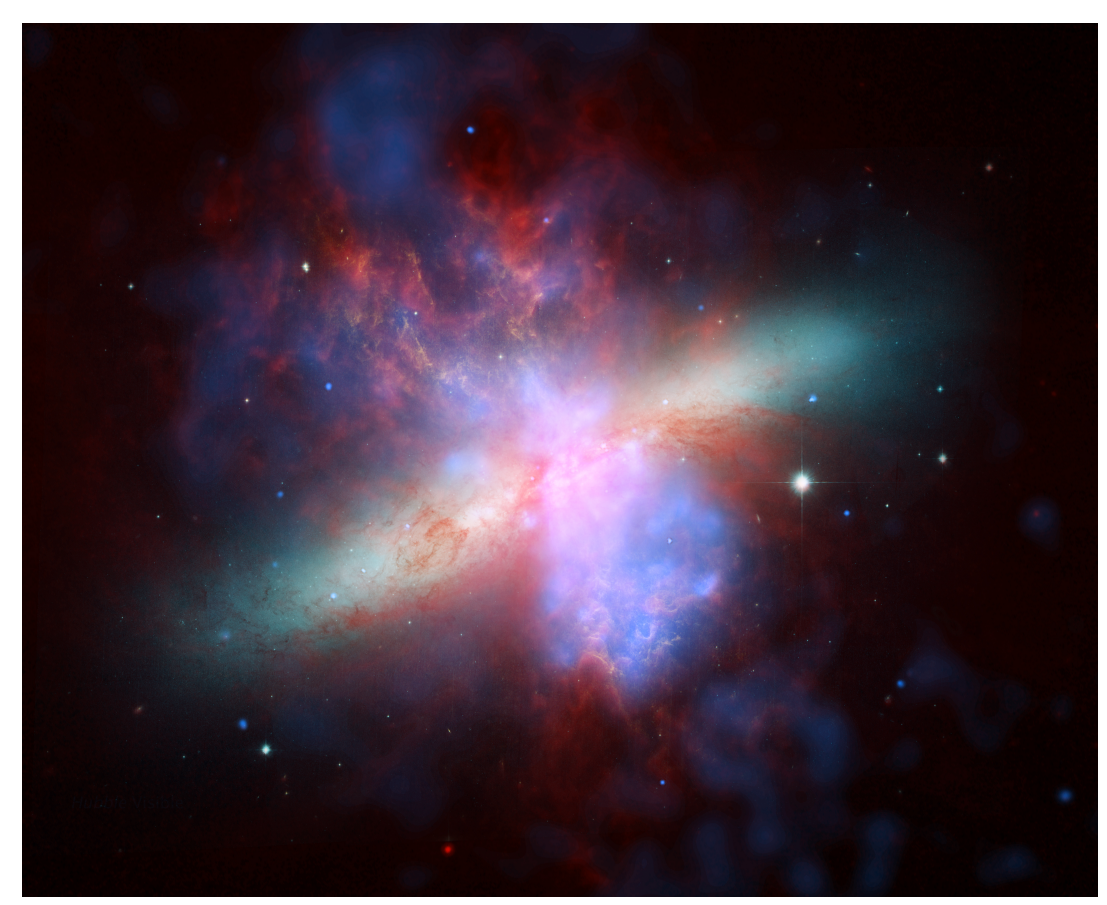

Figure 1.3: M82 is a Starburst Galaxy. The wind generated by SN-driven shocks in its center generates the outflow seen above and below the disc. While the disk is seen in the optical (green and yellow), emitted by starlight, the bipolar cone is composed of hot gases, which emit on X-ray (blue), and is superposed with cold structures emitting in infrared (red) (Geraissate, 2010).

These SB events drive conical winds from the disk at the nuclear region of the galaxy that propagates outside up to dozens of kpc scales with velocities of the order of $600 \mathrm{~km}$ $\mathrm{s}^{-1}$ (e.g., Melioli et al. (2013)). These galaxies, however, do not show any evidence of having an AGN. Seyferts on the other hand, do harbor AGN, as described before, and most ULIRGs seem to do as well. 


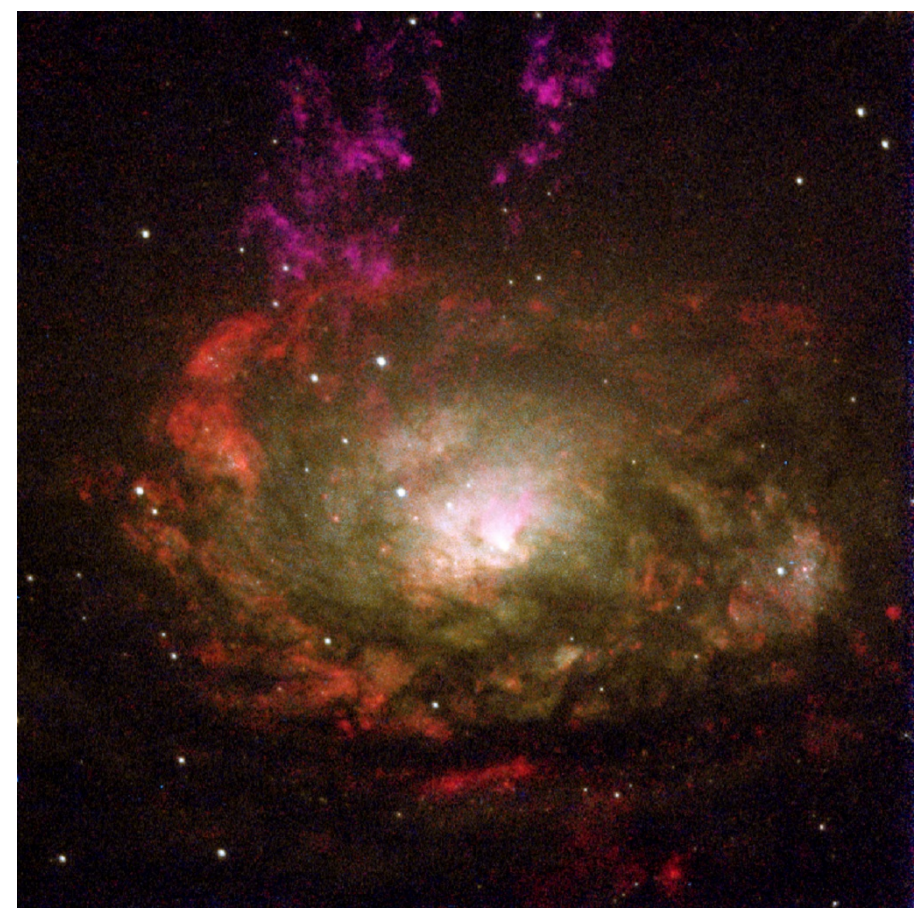

Figure 1.4: The Circinus Galaxy lies 13 million light-years away in the southern constellation Circinus. This galaxy is designated a type 2 Seyfert, a class of mostly spiral galaxies that have compact centers and are believed to contain massive black holes. The black hole and its accretion disk are expelling gas out of the galaxy's disk and into its halo (the region above and below the disk). The detailed structure of this gas is seen as magenta-colored streamers extending towards the top of the image. In the center of the galaxy and within the inner starburst ring is a V-shaped structure of gas. The structure appears whitish-pink in this composite image, made up of four filters. Two filters capture the narrow lines from atomic transitions in oxygen and hydrogen; two wider filters detect green and near-infrared light. In the narrow-band filters, the V-shaped structure is very pronounced. This region, which is the projection of a three-dimensional cone extending from the nucleus to the galaxy's halo contains gas that has been heated by radiation emitted by the accreting black hole. (Maiolino et al. (1998)). Credits, NASA, ESA, and the Hubble Heritage (STSCI/AURA)-ESA/Hubble Collaboration.

ULIRGs are galaxies that are believed to be part of a recent evolutionary merge of galaxies scenario. They have luminosities of $\sim 10^{12} L_{\odot}$, radiating $\sim 90 \%$ of its bolometric luminosity in the infrared. About half harbor simultaneously an AGN and SB activity, this last probably triggered by the compression of clouds during the merge of two galaxies collapsing on their own gravity (Genzel et al. (1998)). Some of them are also classified as Seyfert galaxies (e.g, NGC 6240). ULIRGs present evidence of nuclear and galactic outflows (Armus et al. (1987); Wilman et al. (1999)). In some of these galaxies dominated by star formation, high resolution spectroscopy has revealed outflows with high ejection efficiencies (Rupke et al. (2002)). Furthermore, low velocity outflows are present in ULIRGs dominated by a starburst, while high-velocity outflows are present in systems that show evidence of starburst and AGN (Lípari et al. (2003); Rupke et al. (2005)).

Recently,Tombesi et al. (2015) reported observations of a powerful accretion-disk wind with a velocity of $0.25 c$ in the X-ray spectrum of IRAS F111191+3257, a nearby $(z=$ 
0.189) ULIRG hosting a powerful molecular outflow (Veilleux, S. et al. 2013). The active galactic nucleus is responsible for about 80 percent of the emission, with a quasar-like luminosity of $1.5 \times 10^{46} \mathrm{ergs}$ per second (Veilleux et al. (2013)). The energetics of these outflows is consistent with feedback by the SMBH via radiation pressure (e.g., Zubovas and King (2012b), Faucher-Giguère and Quataert (2012)), see more below.

Accreting SMBHs and star formation strongly influence their host galaxies through feedback, leading to a coevolution of the SMBHs with their hosts. The feedback mechanisms remain under debate (see below), but outflows have been recognized to play a key role in this co-evolution. The examples above show how both UFOs and molecular outflows have an important role in the galaxy evolution, transporting energy and gas outwards from the central regions and also carrying metals to regions of galactic scales. In what follows, we describe the main properties of these outflows.

\section{UFOs and WAs}

Some Seyfert galaxies exhibit regions of intense star formation and highly ionized structures outflowing from parsec to kiloparsec scales at velocities ranging from $100 \mathrm{~km} \mathrm{~s}^{-1}$ up to $100.000 \mathrm{~km} \mathrm{~s}^{-1}$. A systematic study developed by Tombesi et al. (2010, 2011) using the results of the archival search of bright AGNs from XMM-Newton give us new insights in the characteristics of these outflows. These studies show statistical evidence of blueshifted Fe $\mathrm{K}$ absorption lines in 15 of 42 radio-quiet objects. Identification with FeXXV or FeXXVI resonance absorption lines imply UFO velocities up to $\sim 0.3 c$ (Figure 1.5). Recently, observations collected by XMM-Newton and statistical analysis of low redshift AGN Seyfert galaxies in the X-ray band (Tombesi et al. (2013)), with the Chandra and Suzaku satellites, also revealed absorption and emission lines of Fe XXV and Fe XXVI with blueshifts corresponding to velocities between $10.000-100.000 \mathrm{~km}$ $\mathrm{s}^{-1}$. These outflows are known as ultra-fast outflows (UFOs) and are observed near to the central region of its host galaxy between $\sim 1 \mathrm{pc}$ and $\sim 100 \mathrm{pc}$ with column densities $N_{H} \sim 10^{22}-10^{24} \mathrm{~cm}^{-2}$ and ionization parameter ${ }^{2} \log \xi \sim 3-6 \mathrm{erg} \mathrm{s}^{-1} \mathrm{~cm}$ (see also Kraemer et al. (2018)).

In addition, another type of highly ionized outflows were also detected in soft X-ray band, with blueshifts between $100 \mathrm{~km} \mathrm{~s}^{-1}$ to $1000 \mathrm{~km} \mathrm{~s}^{-1}$. These outflows are referred to as a warm absorbers (WAs). WAs reveal multiple narrow absorption lines corresponding commonly to O VII-VIII, Ne IX-X and Fe L. The values of the ionizaton parameter are typically in the range of $\log \xi \sim 0-3 \mathrm{erg} \mathrm{cm} \mathrm{s}^{-1}$, the column densities ranges from $10^{20} \mathrm{~cm}^{-2}$ up to $10^{22} \mathrm{~cm}^{-2}$ and are observed at $\sim \mathrm{kpc}$ scales from the nuclear region (see Tombesi et al. (2013) and references there in). Also, measurements of outflow velocity, column

\footnotetext{
${ }^{2}$ The ionization parameter is given by $\xi=L_{i o n} / n_{H} r^{2}$ where $L_{i o n}$ is the ioniziting luminosity of the AGN, $n_{H}$ is the hydrogen number density and $r$ is the radial distance from the central region. $\log \xi \sim$ 3-6 erg $\mathrm{cm} \mathrm{s}^{-1}$ and temperatures of $\sim 10^{6}-10^{8} \mathrm{~K}$
} 
density, ionization parameter and mass outflow rate in both UFOs and WAs (Tombesi et al. (2013)) result in strong correlations: the closer to the nuclear region, the higher the outflow velocity, ionization parameter and column density, suggesting that UFOs and WAs can be part of the same outflow (Figure 1.6, see also Table 1.1). Figure 1.7 shows an example of UFOs.

a.

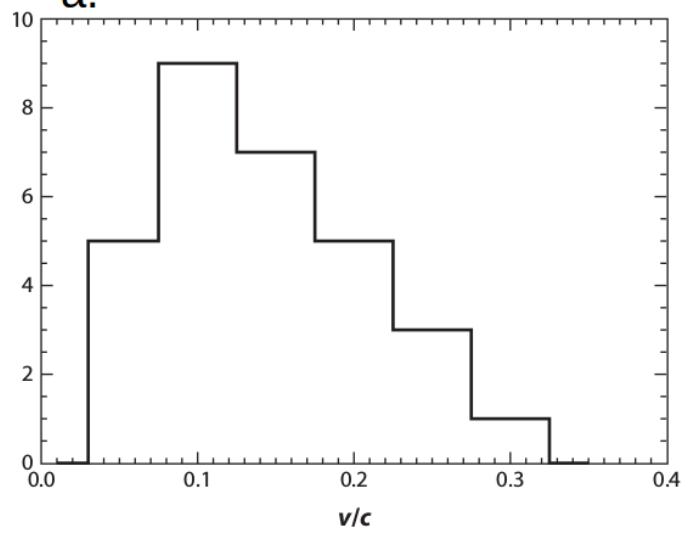

C.

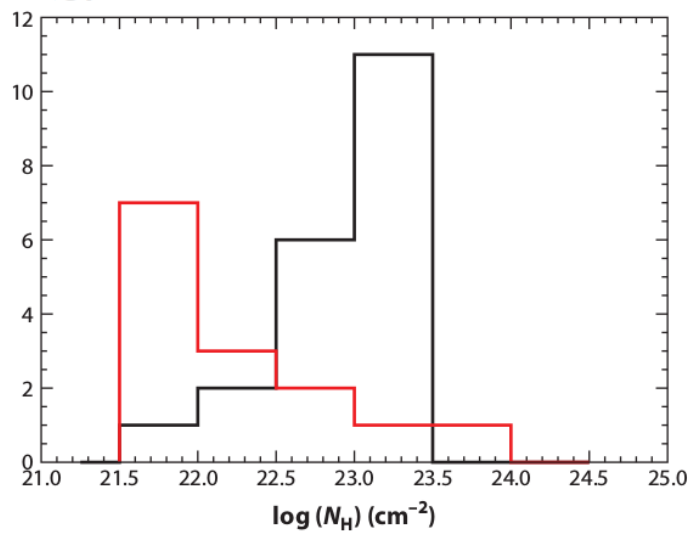

b.

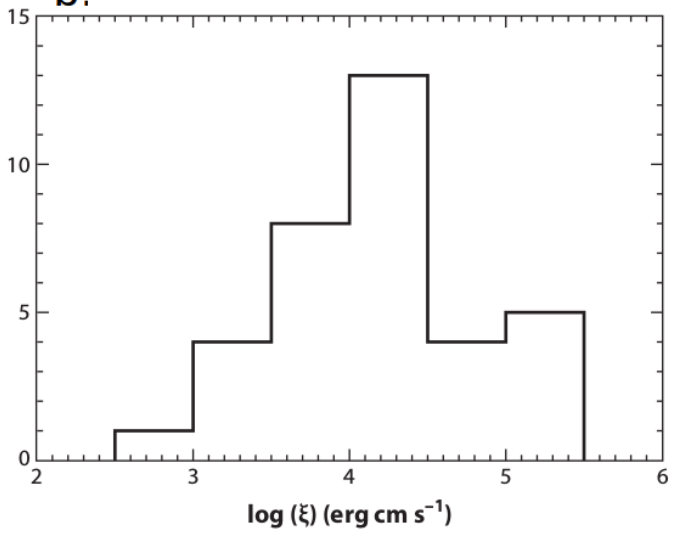

Figure 1.5: Distribution of (a) outflow velocities, (b) ionization parameter and column density in UFOs obtained from modeling the individual spectra from extended observations of a sample of AGN sources in XMM-Newton and Suzaku data arquives (Tombesi et al. (2011), Gofford et al. (2013)). The red-lined histogram in (c) refers to lower limits in column density.

Table 1.1: Comparative table for some properties of WAs and UFOs

\begin{tabular}{ccc}
\hline & Warm Absorbers (WAs) & Ultra-fast Outflows (UFOs) \\
\hline fraction & $>50 \%$ & $>40 \%$ \\
Ions & O VII-VIII, Ne IX-X, Fe L & Fe XXV-XXVI \\
$\ln \xi$ & $0-3 \mathrm{erg} \mathrm{s}^{-1} \mathrm{~cm}$ & $3-6 \mathrm{erg} \mathrm{s}^{-1} \mathrm{~cm}$ \\
$\mathrm{~N}_{H}$ & $10^{20}-10^{22} \mathrm{~cm}^{-2}$ & $10^{22}-10^{24} \mathrm{~cm}^{-2}$ \\
$v_{\text {out }}$ & $\sim 100-1.000 \mathrm{~km} \mathrm{~s}^{-1}$ & $\sim 10.000-100.000 \mathrm{~km} \mathrm{~s}^{-1}$ \\
Origin & tour, outer disk? & inner accretion disk? \\
\hline
\end{tabular}



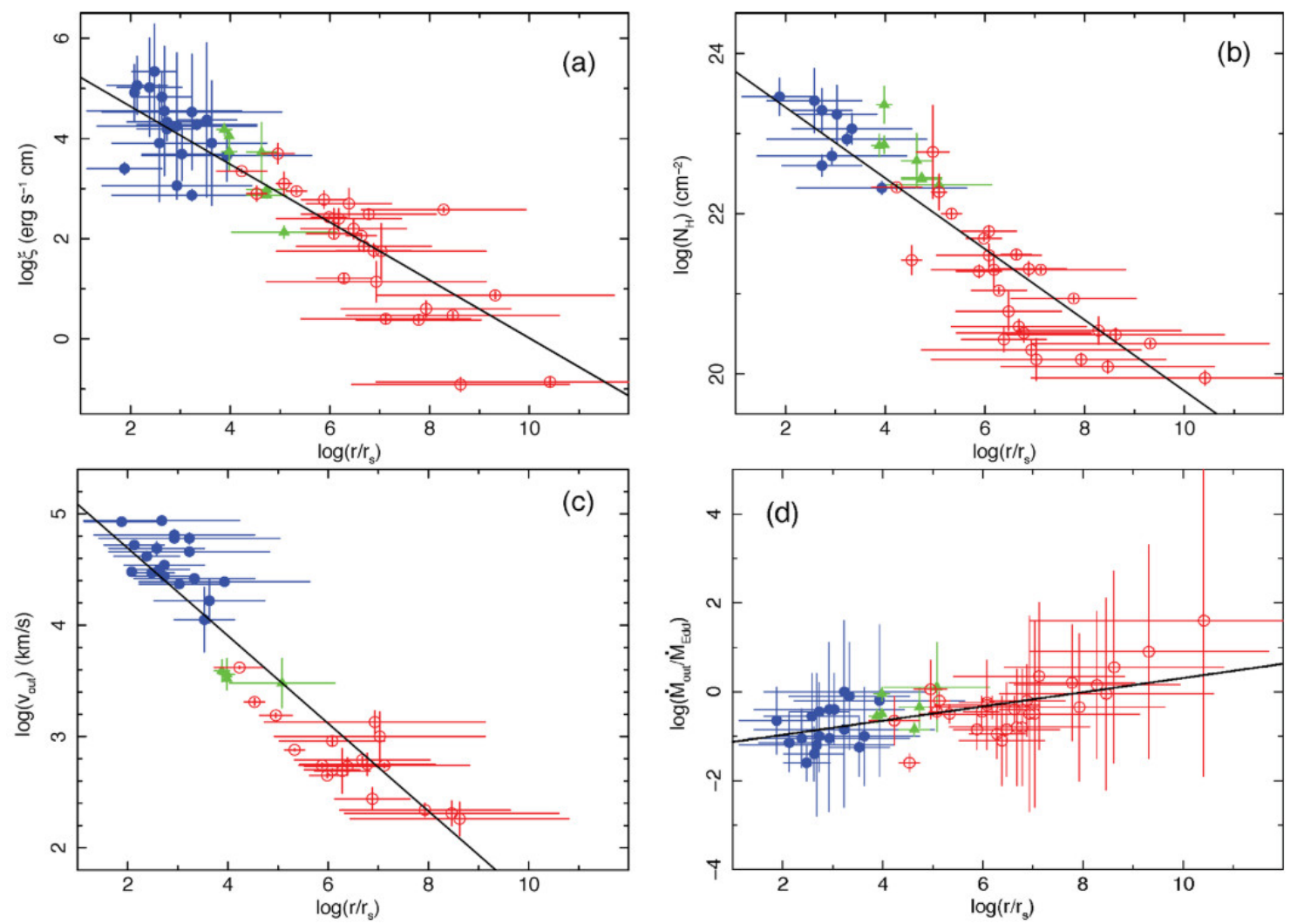

Figure 1.6: Relations between the line-of-sight projected distance $\log r / r_{s}$ (with $r_{s}$ the Schwarzchild radius) and ionization parameter $\log \xi$ (panel a), column density $\log N_{H}$ (panel b), outflow velocity $\log v_{\text {out }}$ (panel c) and mass outflow rate $\log \left(\dot{M}_{\text {out }} / \dot{M}_{E d d}\right)$ (panel d). Red points represent WAs, blue points represent UFOs, and green points non-UFOs. The solid lines indicate the best-fitting linear regression curves (Tombesi et al. (2013)).

\section{Molecular Outflows}

Less energetic than the UFOs, but more massive outflows have been discovered from the study of atomic neutral HI and molecular gas in several active galaxies (e.g. Morganti et al. (2005a); Morganti et al. (2013); Tadhunter et al. (2014)). These molecular outflows typically have extensions of a few $\sim 100 \mathrm{pc}$, column densities $N_{H} \sim 10^{21}-10^{22} \mathrm{~cm}^{-2}$, velocities $\sim 600-1500 \mathrm{~km} \mathrm{~s}^{-1}$, mass outflow rates $20-100 \mathrm{M}_{\odot} \mathrm{yr}^{-1}$ and temperatures $<1000 \mathrm{~K}$. These winds may also have clump structures observed in HI with masses $>600$ $\mathrm{M}_{\odot}$.

An example of a molecular outflow associated with an ULIRG (which is also a Seyfert galaxy) is the source 4C 12.50 shown in Figure 1.8. An outflow of neutral hydrogen (HI) observed in absorption superimposed to a molecular CO outflow with $v \sim 1000 \mathrm{~km} \mathrm{~s}^{-1}$ is co-spatial with the radio jet of this source, suggesting that the molecular outflow is formed out of the radiative cooling of shocked gas by the interaction of the jet with the surrounding interstellar matter of the galaxy (Dasyra and Combes (2012); Morganti et al. (2013), Tadhunter et al. (2014)).

Another example of a molecular outflow is in the radio-loud Seyfert galaxy IC 5063 


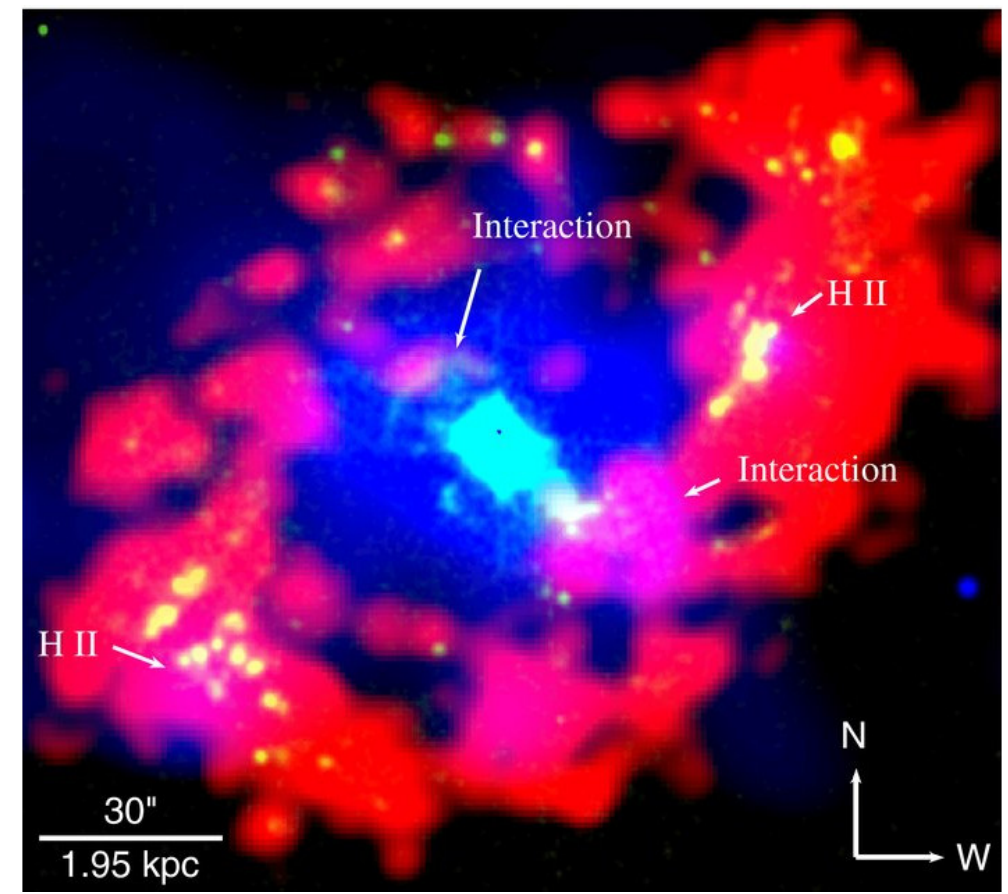

Figure 1.7: This composite image shows the central region of the spiral galaxy NGC 4151, named the "Eye of Sauron". In the "pupil" of the eye, X-rays (blue) from the Chandra X-ray Observatory are combined with optical data (yellow) showing positively charged hydrogen (" $\mathrm{H}$ II") from observations with the 1-meter Jacobus Kapteyn Telescope on La Palma. Two X-ray clumps are present at the terminals of the radio-jet (light-blue) (marked as "interaction"), where the outflow (UFO) appears to encounter dense materials (CO gas lane in the NE and HI clump in the SW). The red around the pupil shows neutral hydrogen detected by radio observations with the NSF's Very Large Array. This neutral hydrogen is part of a structure near the center of NGC 4151 that has been distorted by gravitational interactions with the rest of the galaxy and includes material falling towards the center of the galaxy. The yellow blobs around the red ellipse are regions where star formation has recently occurred (Wang et al. (2010)).

which show what appears a stage in the evolution of galaxy outflows: cold molecular outflows located at $\sim 0.5 \mathrm{kpc}$ with velocities up to $\sim 700 \mathrm{~km} \mathrm{~s}^{-1}$ seem to be powered by a young jet as in 4C 12.50, (Dasyra and Combes (2012)). It has been suggested that the mechanism that preserves the cold temperature is probably dominated by the dust component which can keep the temperature low enough even after the heating of the gas by the shock impinged by the jet.

As stressed, the collimated relativistic jets observed in these sources have been suggested to play a dominant role in driving these outflows at least at sub kiloparsec region, as the observations suggest (e.g, in the sources IC 5063, 3C236), and as we saw above, in some cases (Morganti et al. (2005b); Morganti et al. (2013); Tadhunter et al. (2014); Morganti et al. (2015), Oosterloo et al. (2000)), the location of the molecular outflow appears to be co-spatial with features of the jets hotspots. It is possible that this interaction between the jet and the outflow is enhanced when the enviroment is populated with clumpy structures in the ISM and the jet has a low power. Whether this is a common characteristic it is an open question and is one the motivations for the present work (see 


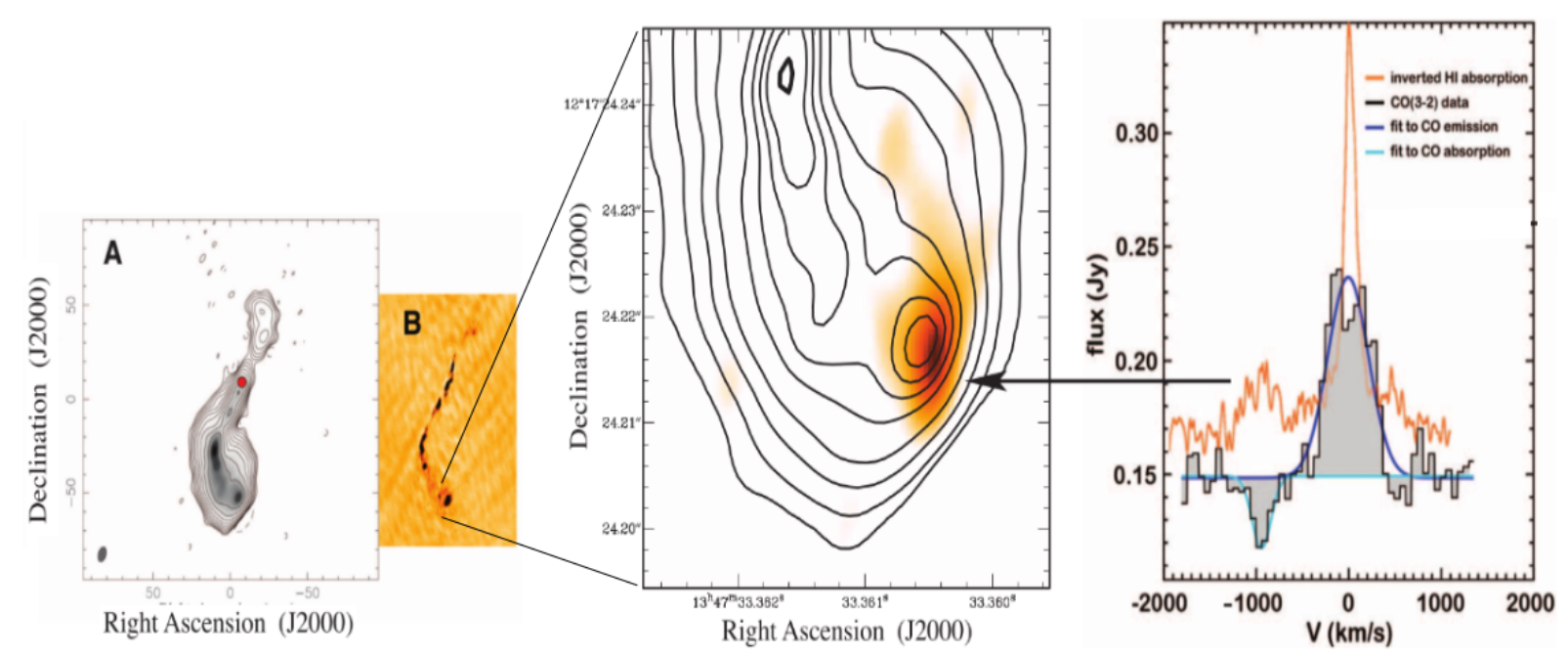

Figure 1.8: Radio continuum images of $4 \mathrm{C} 12.50$ illustrating the morphology of the radio jet (A and $\mathrm{B}$ at the left). (A) The continuum image derived from VLBI data. The red dot indicates the location of the radio core. (B) Continuum image taken from a previous, higher-spatial resolution study (Lister (2003)). The bent structure of the jet and the terminal hot spot in the southern lobe are clearly seen. This hot spot is characterized by an extremely high ( $60 \%)$ fractional polarization. A and B images are displayed on approximately the same scale. At the middle, is shown the distribution of the $\mathrm{HI}$ in two velocity channels showing the location of the two clouds of $\mathrm{HI}$ detected in absorption (orange-white) superimposed on the continuum of $4 \mathrm{C} 12.50$ (contours). At right, the integrated $\mathrm{HI}$ absorption profile is shown in the middle superimposed on the $\mathrm{CO}$ profile [taken from Dasyra and Combes (2012) with the HI from Morganti et al. (2005b) and inverted for comparison]. The northern HI cloud is at the systemic velocity (Morganti (2004)) whereas the southern one is blueshifted $\sim 1000 \mathrm{~km} \mathrm{~s}^{-1}$ relative to the systemic velocity. The location of the southern unresolved cloud producing the blueshifted HI absorption is cospatial with the bright radio hot spot. A diffuse HI component is also observed.

below). Despite the observational and systematic statistical studies developed in recent years, the mechanisms which drive these families of outflows (UFOs, WAs, molecular HIwinds), are still matter of debate. There is a general consensus that the SMBH has a key role in driving these outflows, but the products of star-formation, like the supernova explosions may drive starburst winds that can be also competitive (Melioli and de Gouveia Dal Pino (2015)), particularly in Seyfert galaxies. As stressed before, these are systems where starbursts and star formation regions coexist with the AGN, and where the AGN emits an amount of energy comparable to that of the star formation regions.

Although several studies have been exploring the outflow problem, it seems that the puzzle is far from being solved. The complexity to give a unified model able to explain these outflows including the phenomena the scales, from the central engine $(\sim 1-10 p c)$ to the outskirts of the galaxy and the ICM, is a challenge. 


\section{3 ¿How does the AGN transmit the energy?}

\subsubsection{Radiative Mode}

Up to now, we did not say much about the origin of these outflows. ¿What is the mechanism that triggers these outflows and how these structures are transported to the outer kiloparsec regions of the galaxy?

The literature claims that there are at least two main modes in which the SMBH can release its binding energy to the environment. The stronger (in principle) is the radiation. Nevertheless almost all the radiation escapes freely from the AGN, suggesting that radiation is probably not the principal mechanism affecting its host galaxy (King and Pounds (2015)). In effect, the coupling between photons and the interstellar gas is expected to be very weak if only the Thomson cross section is implied (e.g., Ciotti and Ostriker (2001)), but this coupling is enhanced in the presence of dust. Dust grains are able to scatter and absorb UV radiation, re-emitting it into infrared, but this last radiation escapes almost freely because the dust is optically thin at these frequencies. Thus, since the gas is coupled to the grains hydrodynamically, radiation pressure on the dust may be able to push the gas against its self-gravity, providing a driving to gas outflow.

When momentum from stellar radiation pressure and radiation pressure on larger scales via the light that escapes from star-forming regions is considered (see Hopkins et al. (2011), Hopkins et al. (2012)), a more realistic multiphase ISM develops, maintaining a reasonable fraction of the ISM at densities where the thermal heating from SNe has a larger effect. The potential importance of radiative momentum feedback has been explored for some time now (see, e.g., Haehnelt (1995); Ciotti and Ostriker (2007)), and its potential role in the context of AGNs and SBs was first pointed out by Murray et al. (2005) and Thompson et al. (2005). It might be considered, in principle at least, in some situations to study the evolution of the gas of a galaxy hosting an AGN.

In this mode, likely linked to high Eddington ratios ${ }^{3} L / L_{E d d}>0.1$, the AGN power is believed to couple directly to the ISM of the host galaxy via radiation pressure exerted by the accretion disk winds, resulting in fast outflows (e.g., Aalto et al. (2012); Cicone et al. (2014); Genzel et al. (2014); Tombesi et al. (2015)). The general conclusions of these studies are that AGN feedback can unbind a large fraction of the baryons provided that a few percents of the bolometric luminosity (which is the total luminosity of a source integrated over all wavelengths) couples thermally to the ISM (see, e.g., King (2003);

\footnotetext{
${ }^{3}$ The Eddington ratio is the ratio between the released luminosity and accreting luminosity by the $\mathrm{SMBH} . L_{E d d}$ is the Eddington luminosity which is the maximum luminosity a body (such as a star) can achieve when there is a balance between the force of radiation acting outward and the gravitational force acting inward. The Eddington luminosity is given by $L_{E d d}=4 \pi G M m_{e} c / \sigma_{T}$, where $G$ is the gravitational constant, $m_{e}$ is the electron mass, $c$ is the light velocity and $\sigma_{T}$ is the Thomson cross section defined as a measure of the probability that photons interact with matter by a particular process. When the energy of each individual photon $(h \nu)$ is much smaller than the rest the energy of the electron (its mass times the velocity of light squared, $m c^{2}$ ), the scattering of photons is described by the Thomson cross-section.
} 
Ostriker et al. (2010); Faucher-Giguère and Quataert (2012); Zubovas and King (2012a)).

\subsubsection{Mechanical Mode}

The other form in which the SMBH can communicate its binding energy to the environment is mechanical and probably occurs at low Eddington ratios $\left(L / L_{E d d}<0.1\right)$ and is expected to operate through heat injection into the surrounding gas by a jet (and by starburst-driven winds, as will see). The jet is a highly collimated flow of particles outflowing from the center of the galaxy from a very compact region within parsec scales, transporting energy, momentum and magnetic flux from the inner to the outer regions of the galaxy, affecting the interstellar medium (ISM) and the intergalactic medium (IGM). The electromagnetic output of the jet is prominently in the radio band, which gives the name of radio-mode to the mechanical mode. Jets are probably produced by a magnetocentrifugal process in which plasma is accelerated along the poloidal magnetic field lines that arise from the rotating accretion disk around the BH (e.g., Blandford and Payne (1982); see e.g., de Gouveia Dal Pino (2005); Pudritz et al. (2012) for reviews), or by a process first proposed by Blandford and Znajek (1977) where energy is extracted by the magnetic field directly from the BH spin (e.g. Tchekhovskoy (2015) for a review). The jet exhibits some remarkable features like the presence of internal shocks and hotspots (which are terminal bow shocks formed where the jet impinges on the environment supersonically), which are locations for acceleration of particles to relativistic velocities (e.g., de Gouveia Dal Pino (2005)). It is common to see jets extending up to megaparsec scales like in the Cygnus galaxy which is a clear example of the transport of energy and matter over very large scales (see Figure 1.9).

The feedback of the jet over megaparsec scales transport of hot gas, balancing the temperature and contributing to the reionization of the IGM and ICM (see Krawczynski and Treister (2013) for a review).

At kiloparsec scales $(\sim 1 \mathrm{kpc})$, mainly at the nuclear regions of the host galaxy the importance of the mechanical jet feedback is not fully understood yet and there has been extensive modeling in the literature aiming at providing a better understanding of this process (see e.g., Melioli and de Gouveia Dal Pino (2015) and references therein). In particular, the high degree of collimation of the jet constrains the interaction with the host galaxy environment to a very narrow channel. 


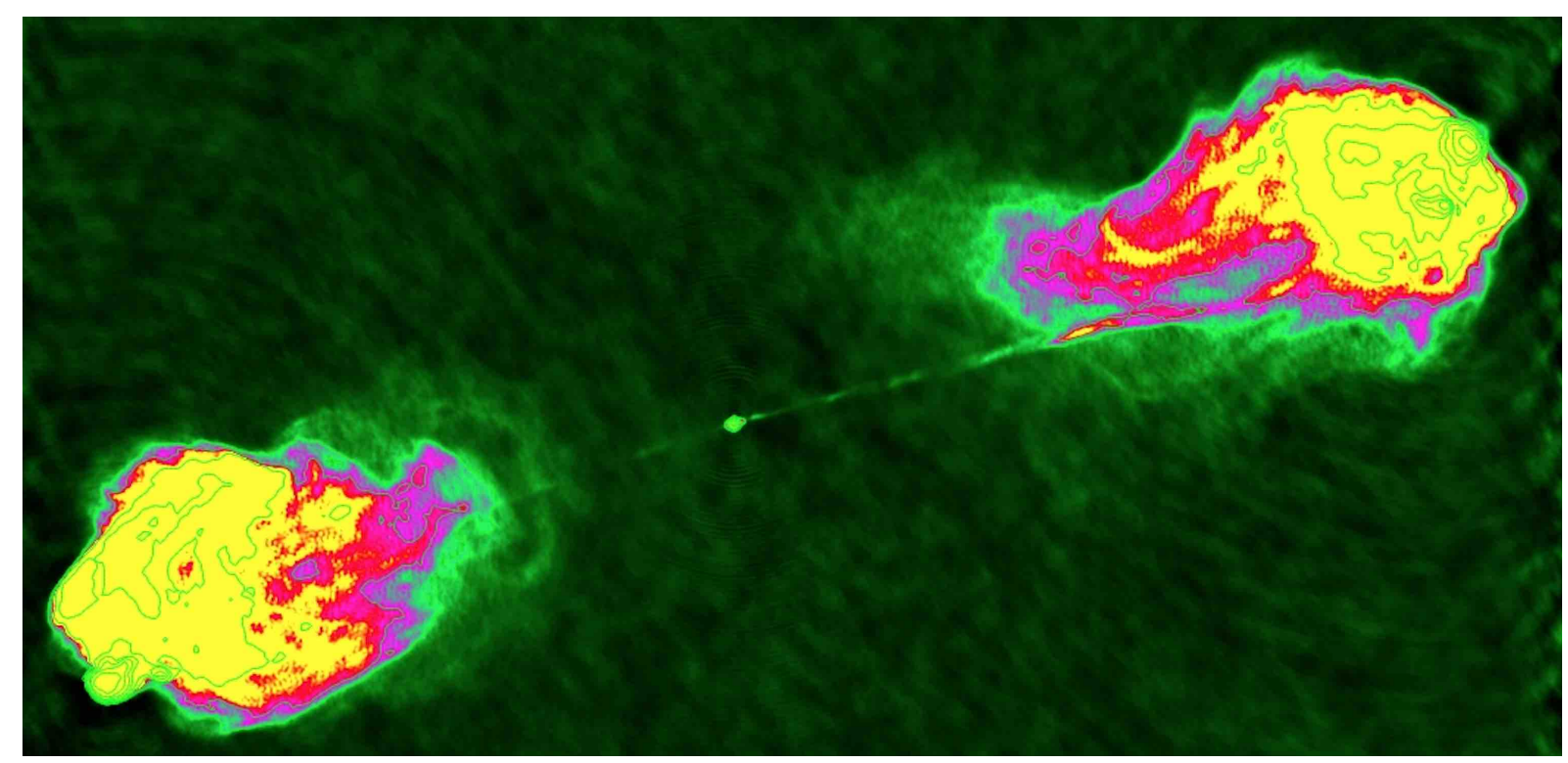

Figure 1.9: Very Large Array radio image at $5 \mathrm{GHz}$ of the first identified extragalactic radio source, Cygnus-A (3C 405) (e.g., Krause (2018)). The color scale was adjusted to emphasize the faint jets. Contours reveal the bright hotspots. The total linear extent is $\sim 120 \mathrm{kpc}$. Radio map provided by Carilli et al. (1989)

\subsubsection{Another mechanical mechanism: Winds induced by su- pernovae $(\mathrm{SNe})$}

\section{Supernovae Remnants (SNR)}

When a massive star explodes, enormous amounts of energy and matter are released into the interstellar medium. The environment is heated and enriched with the material fabricated in the interior of the star over million years. This event is known as a supernova (SN). In this study (see below, and Chapter 2), we will deal with two types of SN:

SN type Ia (SN Ia) is a type of supernova that occurs in binary systems (two stars orbiting around each other) in which one of the stars is a white dwarf. The other star can be anything from a giant star to an even smaller white dwarf. The supernova event, in this case, occurs when the companion star transfers matter to the white dwarf to a threshold amount that causes the burning of the entire star.

SN type II (SN II) results from the rapid collapse and violent explosion of a massive star. Stars with masses between 8 and $40 M_{\odot}$ can suffer this collapse.(for a more detailed definition of SNe, see Ryan and Norton (2010)).

The SN explosion generates a shock front that expands supersonically, sweeping and heating the environment and forming a dense shell which is called supernova remnant (SNR). Inside this SNR, there is hot and rarefied gas. In the initial phase of expansion, called adiabatic phase or Sedov phase the entropy is conserved. At this stage, the SNR shell is not dense enough, so that it expands and sweeps the environment, cooling adiabatically. Later on, when the shock front has swept enough gas from the environment, reaching a 
density large enough as to, it starts to radiate. At this moment the SNR is in a radiative phase. After this phase, the SNR increase even more its density and decrease gradually its temperature. When the dynamical pressure of the SNR equals the external environment pressure, it stops expanding.

\section{Super bubbles}

In galaxies that host a starburst (SB), the star formation rate can be bigger than in normal galaxies (almost 20 times larger. Such galaxies are denominated starburst; see Figure 1.3). The more massive stars will explode as SN II and the SNR formed by each one of them can melt and form a big SNR or a super bubble. Depending on the injected energy and the density of the ISM, one or more, and its internal thermal pressure can reach a radius larger than the thickness of the host galactic disk and, if powerful enough can drive outflows from the disk of the galaxy. For a detailed review of analytical and numerical studies of the formation of galactic winds driven by SNe, we refer to Geraissate (2010), and Melioli et al. (2013), and references therein).

A natural question is, what are the energetic conditions under which a wind can be driven by SNe?

Considering the conditions typical for a Seyfert galaxy, Melioli and de Gouveia Dal Pino (2015) compared the injected energy power by SN Ia and SN II with the radiative energy losses rate by the interstellar gas (due to Bremsstrahlung and recombination processes). This is depicted in Figure 1.10 as a function of the SFR. We see that for a cooling function with a value of $10^{-23} \mathrm{erg} \mathrm{s}^{-1} \mathrm{~cm}^{-3}$, which is a lower limit for a gas at $T=10^{4}$ $\mathrm{K}$, the rate of energy injected is larger than the rate of energy radiated away only for a SFR smaller than $\sim 0.1 \mathrm{M}_{\odot} \mathrm{yr}^{-1}$, and for a value of $10^{-22} \mathrm{erg} \mathrm{s}^{-1} \mathrm{~cm}^{-3}$, which is an upper limit for a gas at $T=10^{4} \mathrm{~K}$, the rate of energy injected is larger than the lost energy rate only for an SFR smaller than $0.01 \mathrm{M}_{\odot} \mathrm{yr}^{-1}$. Above these SFRs, the SFR increases more slowly than the square of the density and therefore there is a critical gas density value above which an outflow cannot develop because of the radiative cooling (see Kennicutt (1998), as indicated in Figure 1.10. This result gives a base to parameterize the energy, SFR and mass injections for the SF-wind before the injected energy is cooled radiatively.

In summary, the processes described above indicate that the feedback and the formation of UFOs and molecular outflows can be driven by radiation pressure and/or the mechanical interaction of the radio jet with the ISM of the host galaxy, or even by a magnetohydrodynamical mechanism is driven by the AGN SMBH (Kraemer et al. (2018)), though the relative role or the efficiency of these mechanisms are not fully understood yet. On the other hand, in such complex systems where the AGN coexists with intense star formation (SF) activity, it is also unclear yet whether the SF, the AGN, or both are driving the outflows (e.g., Storchi-Bergmann et al. (2009), Storchi-Bergmann et al. (2010); Morganti et al. (2013); Melioli and de Gouveia Dal Pino (2015)). In particular, 


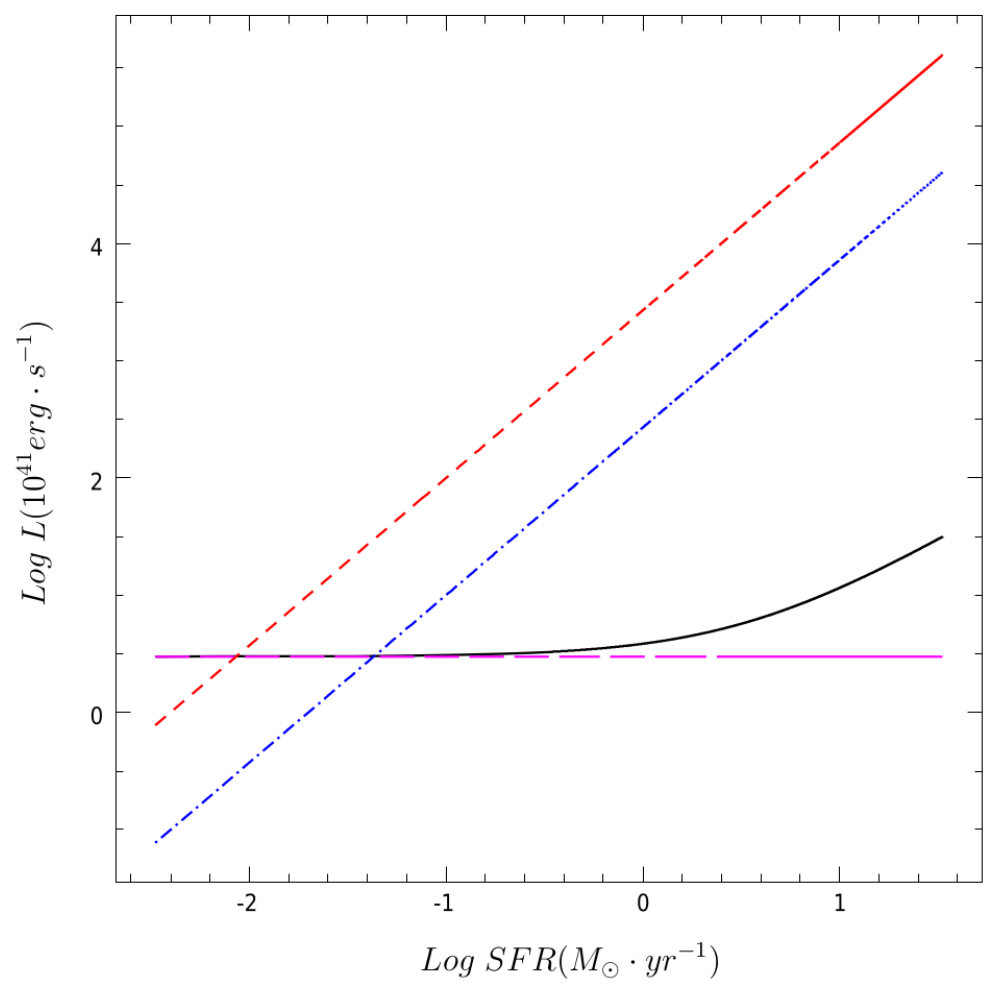

Figure 1.10: Rate of energy injected by $\mathrm{SNe}$ and lost by radiative cooling in the nuclear region of a Seyfert galaxy considering an active region with cylindrical geometry characterized by a radius $\mathrm{R}=300 \mathrm{pc}$ and a height $\mathrm{h}=200 \mathrm{pc}$ as a function of the SFR. Solid (black) line: energy injected by SNe Ia and SNe II; long-dashed (magenta) line: energy injected by the SNe Ia only; dashed (blue) line: energy lost by radiative cooling assuming a value for the radiative cooling function $\Lambda(T)=10^{-23} \mathrm{erg} \mathrm{s}^{-1} \mathrm{~cm}^{-3}$; dot-dashed (red) line: energy lost by radiative cooling assuming a value for the radiative cooling function $\Lambda(T)=10^{-22} \mathrm{erg} \mathrm{s}^{-1} \mathrm{~cm}^{-3}$. The SFR is given in $\mathrm{M}_{\odot} \mathrm{yr}^{-1}$ (log 10 scale), while the energy rates are given in units of $10^{41} \mathrm{erg} \mathrm{s}^{-1}(\log 10$ scale). Extracted from Melioli and de Gouveia Dal Pino (2015)

in Seyfert galaxies, where the nuclear source emits an amount of energy comparable to that of the body of the host galaxy, it would be possible that the outflows are driven by both processes, as remarked before, which therefore would imply that not only the AGN but also SF have a key role in the evolution of the system. In this study, we will consider both, i.e., a Seyfert galaxy which hosts a SB region and and an AGN SMBH as driving sources, and will study their feedback on the evolution of the galaxy and formation of the outflows at the kpc-parsec scale central regions (see below).

In the next section, we will briefly review the current modeling in the literature, based mostly on numerical studies, that have focused on the problem of the feedback and outflow production at galactic scales and then, stress out the motivation of the present work. 


\subsection{On the importance of Magnetic Fields in the study of galaxy outflows}

Measurements of magnetic fields in a sample of around seventy spiral galaxies developed by Beck and collaborators (Beck et al. (2005)) reveals magnetic fields of $\langle\mathbf{B}\rangle=9 \mu \mathrm{G}$. Also, in galaxies with high star formation, the magnetic field intensity has an average of $\sim 30-50 \mu \mathrm{G}$, and of $\sim 100 \mu \mathrm{G}$ near the center, suggesting a relation between star formation and magnetic fields in these galaxies. This makes us believe that the dynamic role played by magnetic fields can result in important phenomena and can give us new insights into unknown important effects in the evolution of galaxy outflows too. For these reasons, we will consider the presence of magnetic fields in this work, since Seyfert galaxies are scenarios which have spiral structures and high star formation rate (in both, Seyferts and ULIRGs). We will take into account magnetic fields of the order of micro-Gauss in order to analyze their effects on the dynamical evolution of outflows at the 1-kpc regions as will see in the following sections.

\subsection{Models of Outflows and Feedback in the litera- ture}

Gaspari et al. (2011a) (Gaspari et al. (2011b), Gaspari et al. (2012); Ciotti (2010); Falceta-Gonçalves et al. (2010a), Falceta-Gonçalves et al. (2010b); Novak et al. (2011)) performed three-dimensional (3D) hydrodynamical simulations of the evolution of the gas in clusters, groups of galaxies, and elliptical galaxies taking into account the AGN feedback, and found that a mechanical feedback with low efficiencies would be enough to both constrain the growth of the black hole and balance the radiative cooling, resulting in gas density and temperature distributions compatible with the observations.

However, these studies, in general, have focused on the large-scale (100 kpc-1 Mpc) evolution of the gas, where most of the energy of the SMBH-jet is directly transferred to the intergalactic or intracluster medium. Almost no work has addressed the effects of the AGN on the host galaxy, where the highly collimated jet has possibly very weak interactions with the surrounding medium. Of course, there is a theoretical interest in the ability of AGNs to affect the gas mass in the nuclear region and its SF history (e.g., Croton et al. (2006); Schawinski et al. 2007), but the inclusion of effects such as the interplay between SF and the AGN activity, the merger of galaxies, and secular processes, in general, is complex, and the parameter space is not yet fully mapped, mainly at small $(1 \mathrm{kpc})$ scales.

In recent works, Wagner et al. (2012) and Wagner et al. (2013) investigated with the help of hydrodynamical (HD) simulations the detailed physics of the feedback mechanism 
between a SMBH jet and a two-phase fractal ISM in the kiloparsec-scale core of a galaxy, but their results refer only to the early stages of the jet evolution, when it propagates through the disk for the first time. Besides, they have assumed a priori existence of very dense, cold clouds spread in the ISM of the host galaxy, without considering the mechanisms that allowed for the formation and evolution of these clouds in the ISM. In this case, the dense embedded clouds of the ISM are accelerated by the ram pressure of the high-velocity flow through the porous channels of the warm-phase gas, but probably this regime cannot be maintained for much time after the jet digs a hole in the disk and beyond it, and therefore this cannot be taken as a stationary solution.

In a concomitant work, Melioli and de Gouveia Dal Pino (2015), have accounted for the relative feedback contribution of both the AGN and the products of SF in the formation of outflows in Seyfert galaxies, also by means of high-resolution 3D HD simulations. They have considered the effects of radiative cooling and, in order to account for the SF feedback, they considered observed SFRs in Seyfert galaxies, and type I and II supernovae explosions that naturally drive starburst winds ((as described in section 1.3.3)). For the AGN feedback, they considered a collimated jet emerging from the central region of the active galaxy. They have also considered the evolution of the ISM of the galaxy starting with a stratified, multi-phase interstellar gas with initial temperatures between $10^{4} \mathrm{~K}$ and $10^{6} \mathrm{~K}$, supported by thermal pressure and rotation against gravity, mimicking the conditions of typical spiral galaxies, as in Seyferts. From the simulations, they computed the gas mass lost by the system, separating the role of each of the injection energy sources on the galaxy evolution, and found that at scales within $1 \mathrm{kpc}$ an outflow can be generally established considering intense nuclear SF only, and the maximum velocities it achieves in this case are around $1000 \mathrm{~km} \mathrm{~s}^{-1}$, thus compatible with those of molecular outflows. However, the derived temperatures in the wind at too high and the densities too low in comparison to those observed in the molecular outflows. The jet alone is unable to drive a massive gas outflow, although it can sporadically drag and accelerate clumps of the underlying outflow to very high velocities comparable to those of UFOs, but it causes a lot of gas fallback, like fountains (Melioli et al. (2008); Melioli et al. (2009)) that decrease the mass outflow to values much smaller than those observed in the molecular outflows.

\subsection{1 ¿What will we do in this work?}

Motivated by the advances and unsolved questions in the studies described above, in this work, we have extended the previous study performed by Melioli and de Gouveia Dal Pino (2015) by adding new physical ingredients. In order to achieve a better understanding of the processes driving the nuclear gas evolution in a Seyfert galaxy, we perform fully 3D hydrodynamical (HD) and magnetohydrodynamical (MHD) simulations with (3.9 pc) resolution, taking into account the effects of magnetic fields, which have been neglected in 
the previous studies of Melioli and de Gouveia Dal Pino (2015) and Wagner et al. (2012), and also the effects of the feedback of AGN winds with large opening angles. This latter must allow for a stronger interaction with the ISM gas than that provided by collimated $\left(0^{\circ}\right.$ opening angle) jets. We should remark that in this study we will consider only the mechanical AGN feedback and neglect its radiation feedback (see also Martin (2005); Sharma et al. (2011)). The photoionizing radiation will be considered only to justify the minimum temperature of the diffuse gas, $T_{\text {min, gas }} \sim 10^{4} \mathrm{~K}$. In fact, we assume that all the mechanical energy and momentum are effectively deposited into the ISM, so that the mechanical feedback will dominate over the radiative feedback. This is a reasonable assumption at least for Seyfert galaxies for which the column densities are about $\sim 10^{22}$ $\mathrm{cm}^{-2}$, as pointed out by Novak et al. (2011). Moreover, although dusty structures, in the form of spirals and filaments at hundreds of parsec scales, are often observed in active galaxies (see, e.g., Simões Lopes et al. (2007)), indicating that a reservoir of dust is a necessary condition for the nuclear activity, in Seyfert galaxies the dust mass is often low enough to suffer destruction in SN-generated shock waves that can make the typical dust lifetime shorter than the time over which the galaxy is active (McKee (1989); Jones and Nuth (2011)). Though this is controversial, we still can neglect the effects of a pressure radiation feedback in our simulations. This due to resolution constraints because we are driving the AGN outflow at parsec scales (see Chapter 3). Thus, assuming that the action of the radiation pressure in driving the outflow has taken place at the smaller sub-parsec scales in the nuclear region, which is not resolved in our computational domain, then the larger scales of our simulations we can take into account only the mechanical feedback of this generated outflow upon the optically thin plasma of the galaxy.

\section{6 ¿How is this dissertation organized?}

This dissertation is organized as follows. In Chapter 2, we will present the set of equations governing the evolution of our physical system and the numerical tools to solve them, as well as the initial and boundary conditions on the computational domain, leaving the description of the numerical code to the Apendice I. In Chapter 3, we will discuss the results of our numerical HD and MHD simulations and compare them with previous results and the observations. Finally, in Chapter 4 we will present the conclusions of this work and our perspectives for future work. 
CHAPTER 2

Numerical Model

In this chapter, we will describe our numerical model for three-dimensional (3D) radiative cooling hydrodynamical (HD) and magneto-hydrodynamical (MHD) simulations of the nuclear region (within $1 \mathrm{kpc}^{3}$ ) of an active galaxy with properties similar to Seyferts (with ULIRGs and UFO properties) aiming to explore the AGN and SF feedbacks in the evolution of these galaxies and their outflows. Here, we will describe the numerical model in detail, including the numerical method and the magnetohydrodynamical equations we integrated, the initial setup and boundary conditions. In Chapter 3, we will present the results of the numerical simulations.

\subsection{Numerical Method}

The equations which describe the dynamics of the system are the MHD equations and are given by

$$
\begin{aligned}
\frac{\partial \rho}{\partial t}+\nabla \cdot(\rho \boldsymbol{v}) & =\dot{m}_{S} \\
\frac{\partial \rho \boldsymbol{v}}{\partial t}+\nabla \cdot\left(\rho \boldsymbol{v} \boldsymbol{v}-\boldsymbol{B B}+\boldsymbol{p}^{*}\right) & =f_{G} \\
\frac{\partial E}{\partial t}+\nabla \cdot\left[\left(E+p^{*}\right) \boldsymbol{v}-\boldsymbol{B}(\boldsymbol{B} \cdot \boldsymbol{v})\right] & =E_{S} \\
\frac{\partial \boldsymbol{B}}{\partial t}-\nabla \times(\boldsymbol{v} \times \boldsymbol{B}) & =0
\end{aligned}
$$


The equations (2.1), (2.2), (2.3) and (2.4), correspond to continuity, momentum, energy and magnetic induction equations, respectively. $\rho$ is the density, $\boldsymbol{v}$ is the velocity, $\boldsymbol{B}$ is the magnetic field, $\boldsymbol{p}^{*}$ is a diagonal tensor with components $p^{*}=p+B^{2} / 2$ and $E$ is the total energy density given by,

$$
E=\frac{p}{\gamma-1}+\frac{1}{2} \rho v^{2}+\frac{B^{2}}{2}
$$

where $p$ the thermal pressure and $\gamma$ is the ratio of the specific heats and was assumed a state equation for a ideal gas, $p=(\gamma-1) e \cdot \dot{m}_{S}$ represents the rate of mass injection by SNe Ia and SNe II, $f_{G}$ represents the external forces due to dark mater, the bulge and the disk and $E_{S}=E_{S N I a}+E_{S N I I}+E_{A G N}+E_{D M}+E_{\text {disk }}+E_{\text {bulge }}-\Lambda(\rho, T)$ represents the sources of energy coming from SN type Ia and SN type II explosions, the AGN wind, the external potential due to drk matter halo, the disk and the stellar bulge as well as the losses by radiative cooling respectively (see Apendix A).

The simulations used to study the AGN and SF feedback in Seyfert galaxies were performed using the AMUN Code (Kowal et.al 2007). In this work, we used a similar version of this code that was modified by Melioli and de Gouveia Dal Pino (2015) in order to introduce the effects of radiative cooling and to build an appropriate setup to describe the environment of a Seyfert galaxy (see details below). The AMUN is a Godunov based code, which integrates the HD and MHD equations in space using the Harten-Laxvan-Leer C (HLLC) Riemann solver for the HD-simulations and Harten-Lax-van-Leer D (HLLD) for the MHD-simulations (Toro (1999)), and a second-order Runge-Kutta scheme (Ketcheson (2008)) is employed for time integration (see Appendix I. for a more detail description of the numerical method). It also uses the message-passing interface library to achieve portability and efficient scalability on a variety of parallel high-performance computing systems.

The code includes a parameterized cooling function calculated implicitly in the energy equation, implemented by Melioli and de Gouveia Dal Pino (2015), that allows the gas to cool down to temperatures of the order of $100 \mathrm{~K}$, with errors smaller than $10 \%$, and which is calculated implicitly in each time step for each grid position. The cooling function considers an optically thin gas in ionization equilibrium and takes into account the gas metallicity and the gas fraction of molecular hydrogen $H_{2}$, according to the methodology used by Raga et al. (2000), Raga et al. (2002).

\subsection{Computational Domain and Boundary Conditions}

The adopted box for the computational domain has physical dimensions of $1 \times 1 \times 1$ $\mathrm{kpc}^{3}$ in the $\mathrm{x}, \mathrm{y}$, and $\mathrm{z}$ directions, respectively. We have run all the models with a maximum resolution of 3.9 pc per cell with $256^{3}$ resolution. Tests performed by Melioli and de 
Gouveia Dal Pino (2015) considering also simulations with $128^{3}$ and $512^{3}$ resolutions have indicated a good degree of convergence in the results for $256^{3}$ resolution. For this reason, we have adopted this resolution in the present work.

The energy associated with each SN explosion (see below) is injected as thermal energy in a single cell, while the AGN-wind is injected in four cells above and below the midplane disk, at the center of the system. The total physical simulation time was $\sim 6 \mathrm{Myr}$.

Each simulation was run on 128 processors of the LAI supercomputing center at IAGUSP, using about $10^{5} \mathrm{CPU}$ hr per simulation.

Our simulations we have adopted outflow boundaries. This condition ensures that the fluid reaching the boundaries escapes from the system without coming back. In the case of the magnetic field, we have ensured the divergence free condition in the boundaries.

\section{$2.3 \quad$ Initial Setup}

We adopt here the same initial condions for the galactic system as in Melioli and de Gouveia Dal Pino (2015). For this reason, in the following paragraphs of this section we transcribe most of the equations of section 4 of that work. The initial distribution of the gas in the disk is obtained from the solution of the equation of motion in stationary state $\left(\partial_{t} v_{i}=0\right)$. For a magnetic field at $t=0, \vec{B}=0$, from equation (2.2) and using (2.1) we have,

$$
\left(\partial_{j} v_{i}\right) v_{j}+\frac{1}{\rho} \partial_{j}\left(p \delta_{i j}\right)=-\partial_{i} \Phi
$$

In the midplane $z=0$ of the disk, we assume for simplicity that the gas is initially supported by the rotation of the galaxy in the radial direction. In this way, the rotational velocity, $v_{\phi}$ (the azimuthal component of the velocity of the equation (2.5) above) is given by (see Geraissate (2010)),

$$
v_{\phi}=e_{r o t}\left(\frac{r \partial \Phi}{\partial r}\right)^{1 / 2}
$$

where the term $e_{\text {rot }}$ was introduced in order to make a correction on $v_{\phi}(r)$ due to the presence of other forces in the gas than the centrifugal force. If the disk is only supported against the rotation, we have $e_{r o t}=1$. In a more realistic case (which will be actually considered in the evolution of the system), part of the gravitational force is supported by the thermal and turbulent pressures of the interstellar medium (ISM), as well as by the magnetic forces when magnetic fields are considered. We adopt, as in Strickland and Stevens (2000), $e_{\text {rot }}=0.95$.

The equation above is only valid for $z=0$ at $t=0$, as we stressed before. To attenuate the velocity rotation of the gas above the galactic disk and an initial non-rotating galactic 
halo in our domain we assume a simple model where the rotation falls exponentially with increasing height above (and below) the galactic plane,

$$
e=e_{\text {rot }} \exp \left(-z / z_{\text {rot }}\right)
$$

where $z_{\text {rot }}=5 \mathrm{kpc}$ is the height scale for the reduction by a factor of $e$ of the rotational velocity with height.

In our models, we consider the central $(\mathrm{kpc})$ region of a typical spiral Seyfert galaxy and set the initial conditions for the ISM following the procedure outlined in Melioli et al. (2008), Melioli et al. (2009), Melioli et al. (2013) and Melioli and de Gouveia Dal Pino (2015).

We consider an initial mass for the galaxy which includes the contribution of a bulge ${ }^{1}$ and a stellar disk, and then we set the ISM in equilibrium with the gravitational potential given by the sum of the dark matter halo, the bulge, and the disk contributions. The dark matter halo is assumed to follow the Navarro-Frenk-White profile (Navarro et al. (1996)):

$$
\Phi_{D M}=-\frac{G M_{v i r}}{r_{s} f(c)} \frac{\ln (1+x)}{x},
$$

where $r$ is the spherical radius, $M_{v i r}$ is the mass at the virial radius, $r_{v i r}$ which is defined as the radius where the average density is $\sim 10^{2}$ times the cosmological critical density $\rho_{\text {crit }}$, $r_{s}$ is a scale radius, $x$ is the ratio between $r$ and $r_{s}, \mathrm{c}=r_{v i r} / r_{s}$ and $f(c)=\ln (1+c)-c(1+c)$ . In this model we considered $M_{v i r}=10^{11} M_{\odot}, r_{v i r}=120 \mathrm{kpc}, r_{s}=10 \mathrm{kpc}$, and $c=12$, adopting a $\Lambda$ cold dark matter cosmological universe with matter (barionic plus dark matter) density parameter $\Omega_{M}=0.27$, a dark energy density parameter $\Omega_{L}=0.73$, and a Hubble constant $H_{0}=71 \mathrm{~km} \mathrm{~s}^{-1} \mathrm{Mpc}^{-1}$. We note that owing to the limited extension of the galaxy region investigated here, we verify that the gravitational potential of the dark matter halo has almost no influence in the determination of the initial distribution and the evolution of the gas.

The gravitational potential of the stellar bulge is given by a Plummer distribution (Plummer (1911)):

$$
\Phi_{b}(r)=-\frac{G M_{b}}{r_{c}+r}
$$

where $M_{b}$ is the total mass of the bulge of the galaxy and $r_{c}$ is the core radius, defined as the radius containing $50 \%$ of the stellar mass. The bulge mass is assumed to be always $M_{b}=10^{10} M_{\odot}$. Finally, the gravitational potential of the gas disk is given by a Miyamoto \& Nagai profile (Miyamoto and Nagai (1975)), that is,

\footnotetext{
${ }^{1}$ (i.e., the spherical distribution of stars around the nucleus typically observed in spiral galaxies)
} 


$$
\Phi_{d i s k}(r, z)=-\frac{G M_{d i s k}}{\sqrt{r^{2}+\left(a+\sqrt{z^{2}+b^{2}}\right)}},
$$

where $M_{d i s k}$ is the mass of the disk of the whole galaxy, while $a$ and $b$ are its radial and vertical scales, respectively. In this study we assume $M_{d i s k}=5 \times 10^{10} M_{\odot}, a=8 \mathrm{kpc}$, and $b=0.8 \mathrm{kpc}$.

Therefore, the total gravitational potential is $\Phi(r, z)=\Phi_{d m}+\Phi_{b}(r, z)+\Phi_{\text {disk }}(r, z)$.

Since we are here interested to explore in detail the mass outflows in Seyfert galaxies and the origin of the high velocity and sometimes high-density structures that are observed in such flows, we assume here a galaxy model characterized by a gas disk distribution with high column density $N_{H}=10^{23} \mathrm{~cm}^{-2}$, which is compatible with those observed in galaxies with UFOs and molecular outflows (as described in Chapter 1). For a comparison between models with different initial column densities we refer to Melioli and de Gouveia Dal Pino (2015).

Our model considers a stratified multiphase interstellar gas distribution, each phase "i" is defined by a typical temperature $T_{i}$, initially in hydrostatic equilibrium in the gravitational potential described above. The initial density distribution of each phase is given by

$$
\rho_{i}(r, z)=\rho_{i, 0} \exp \left(-\frac{\Phi(r, z)-e_{i}^{2} \Phi(r, 0)-\left(1-e_{i}^{2}\right) \Phi(0,0)}{c_{s, i}^{2}}\right),
$$

where $\rho_{i, 0}$ is the density of the "i" phase at $z=0, c_{s, i}=\left(k_{B} T_{i, 0} / \bar{m}\right)$ is the isothermal sound speed of the $i$-phase of the gas and $e_{i}$ quantifies the fraction of rotational support of the ISM. The galaxy parameters adopted in this study are presented in Table 2.1 and the initial gas density distribution is shown in Figure 2.1.

Table 2.1: Parameters for the initial setup of the galaxy model

\begin{tabular}{ccccccccccccc}
\hline $\begin{array}{c}r_{c} \\
(\mathrm{kpc})\end{array}$ & $\begin{array}{c}\rho_{1,0} \\
\left(\mathrm{~cm}^{-3}\right)\end{array}$ & $\begin{array}{c}\rho_{2,0} \\
\left(\mathrm{~cm}^{-3}\right)\end{array}$ & $\begin{array}{c}T_{\text {disk }, 1} \\
(\mathrm{~K})\end{array}$ & $\begin{array}{c}T_{\text {disk }, 2} \\
(\mathrm{~K})\end{array}$ & $\begin{array}{c}T_{\text {disk }, 3} \\
(\mathrm{~K})\end{array}$ & $e_{1}$ & $e_{2}$ & $e_{3}$ & $\begin{array}{c}N_{H} \\
\left(\mathrm{~cm}^{-2}\right)\end{array}$ & $\begin{array}{c}M_{\text {Bulge }} \\
\left(M_{\odot}\right)\end{array}$ & $\begin{array}{c}M_{\text {disk }} \\
\left(M_{\odot}\right)\end{array}$ & $\begin{array}{c}M_{\text {vir }} \\
\left(M_{\odot}\right)\end{array}$ \\
\hline \hline \\
\hline \multirow{2}{*}{1.3} & 1 & $10^{4}$ & $5 \times 10^{4}$ & $10^{5}$ & 1 & 0.9 & 0.5 & $2 \times 10^{23}$ & $10^{10}$ & $5 \cdot 10^{10}$ & $10^{11}$ \\
\hline
\end{tabular}

As stressed in Chapter 1, the main driving sources of energy and outflows in the active galaxies are the AGN SMBH and Type I and II supernovae.

The rate of SN Ia explosions is proportional to the bulge mass $\left(M_{b}\right)$, which in turn is proportional to the mass of the central $\operatorname{SMBH}\left(M_{B H}\right)$. Given the relation $M_{B H} / M_{b} \sim 10^{-3}$ (see, Häring and Rix (2004)), and assuming a SN Ia rate in the bulge of $\sim 0.01 \mathrm{yr}^{-1}$ for a $10^{10} M_{\odot}$ bulge mass (Pain et al. (1996)), we obtain a rate of SN Ia in terms of the black hole mass (in solar mass units) 


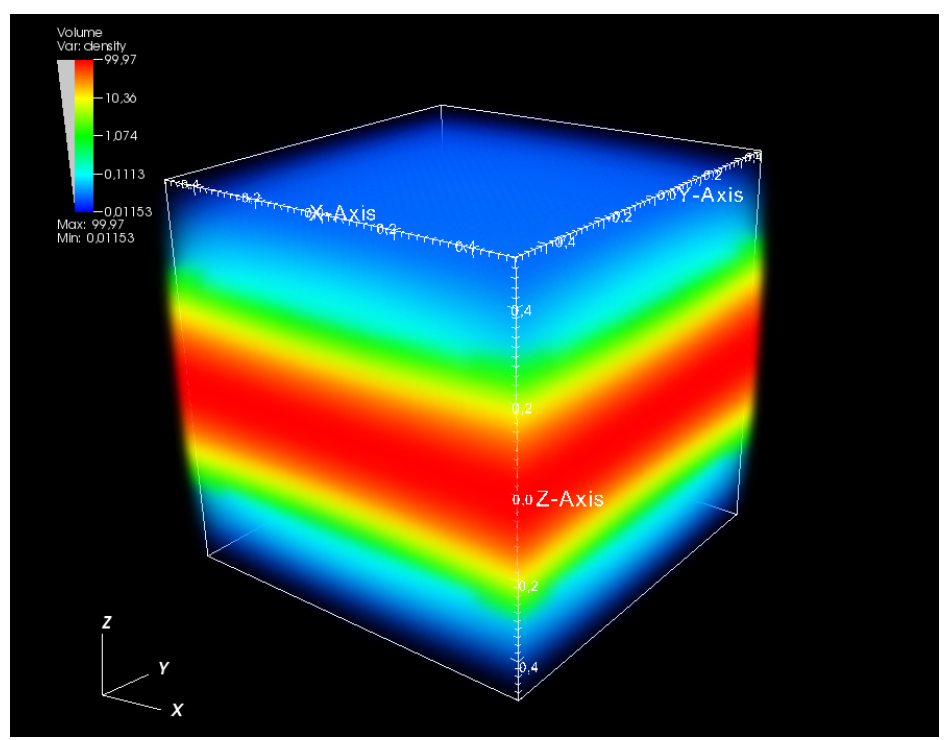

Figure 2.1: 3D initial logarithmic gas density distribution for the central region of the galaxy within $1 \mathrm{kpc}^{3}$. Density is given in $\mathrm{cm}^{-3}$ and distances are given in pc.

$$
N_{S N I a}=10^{-9} M_{B H} y r^{-1}
$$

Each $i$-th SN explosion is associated randomly with a given position $P_{i}$ in the stellar bulge of the galaxy at a random time $t_{i}$ in the interval $0<t_{i}<t_{S F}$, where $t_{S F}=30 \mathrm{Myr}$ is the typical time for stellar activity ${ }^{2}$. This procedure generates points with a random spatial frequency proportional to the stellar density in the bulge, $\rho_{b, s t a r}$, as described by the Plummer profile (Plummer (1911)):

$$
\rho_{b, s t a r}(r)=\frac{3 M_{b}}{4 \pi r_{c}^{3}}\left(1+\frac{r^{2}}{r_{c}^{2}}\right)
$$

As described in Chapter 1, we also consider the occurrance of starbursts (SBs) in the central region of the galaxy driven by the products of late massive star formation, i.e., by type II supernovae (SN II) in a lifetime of $30 \mathrm{Myr}$. Specifically, we consider a SB within a disk radius of $\sim 300 \mathrm{pc}$ and a typical half-height (above and below the disk) of $160 \mathrm{pc}$. In an event, a total mass of stars $3 \times 10^{8} \mathrm{M}_{\odot}$ is produced. Considering that the $\mathrm{SN}$ rate is $10^{-1} \mathrm{yr}^{-1}$ which is in agreement with observations of Seyfert galaxies (e.g., Forbes and Norris (1998)), we expect to have $3 \times 10^{6}$ SNe injecting an average luminosity $L_{S N} \sim 3 \times 10^{42} \mathrm{erg} \mathrm{s}^{-1}$ in a time interval of $30 \mathrm{Myr}$.

As in the case of the SNe I, in order to set the SNe II spatially and temporally within the SB region, we associate randomly with each i-th SN II a position $P_{i}=\left(r_{i}, z_{i}\right)$, where $0<r_{i}<300 \mathrm{pc}$, and $\left|z_{i}\right| \leq 160 \mathrm{pc}$, at a time $t_{i}$ in the range $0<t_{i}<30 \mathrm{Myr}$.

For both the SNe Ia and the SNe II, we inject the energy of each explosion over a time of $300 \mathrm{yr}$ and $L$ the injected and radiated powers, respectively (Melioli and de Gou-

\footnotetext{
${ }^{2}$ Clayton, D.D. Principles of stellar evolution and nucleosyntesis
} 


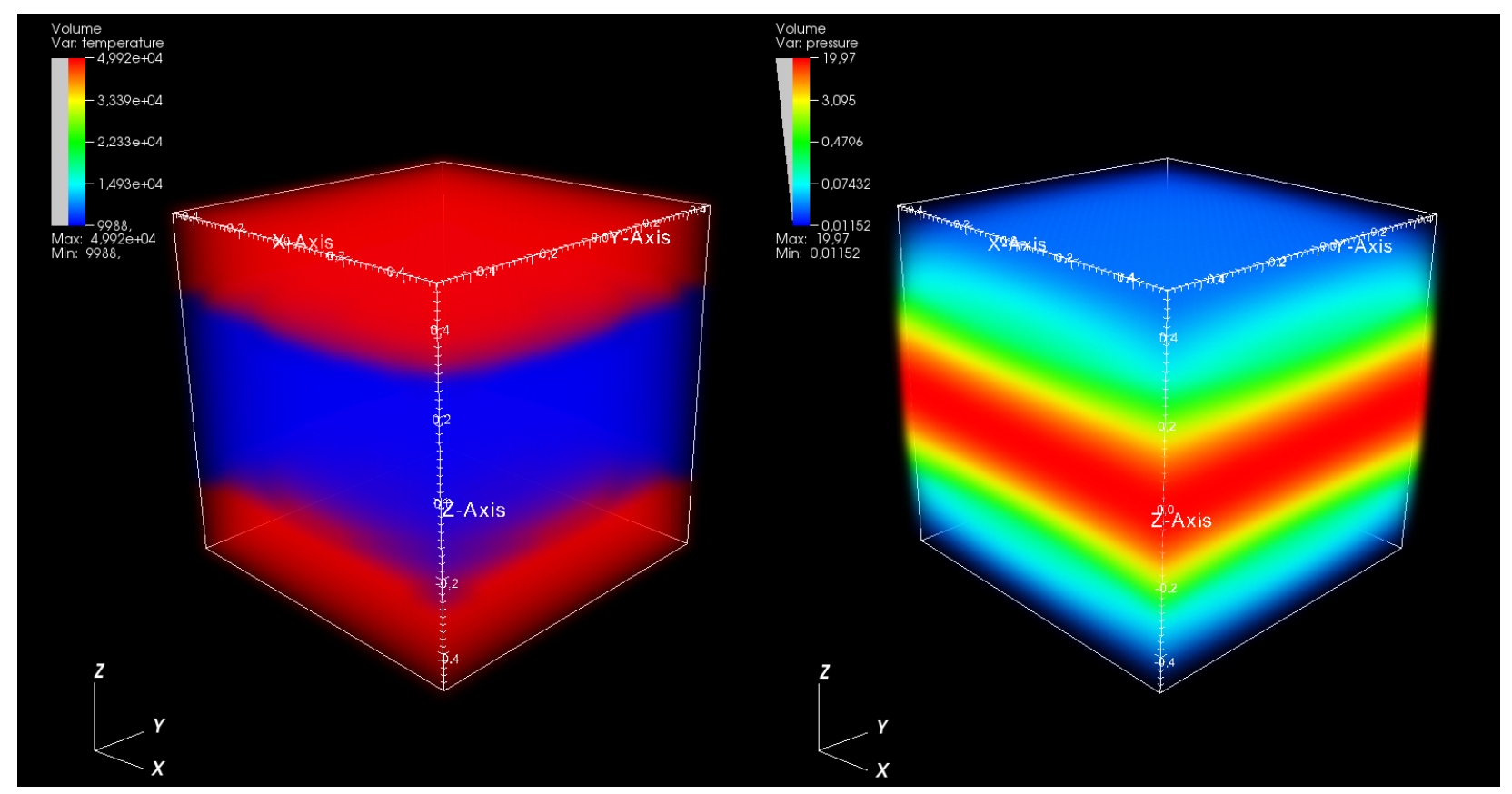

Figure 2.2: $3 \mathrm{D}$ initial logarithmic gas temperature and pressure distribution (left and right, respectively) for the central region of the galaxy within $1 \mathrm{kpc}^{3}$. Temperature is given in $K$, pressure in $\mathrm{erg} \mathrm{cm}^{-3}$ and distances are given in pc.

veia Dal Pino (2004)). Therefore, each SN explosion injects mass and energy at rates $\dot{M}=M_{i n j} /(300 \mathrm{yr})$ and $L_{S N}=10^{51} \mathrm{erg} /(300 \mathrm{yr})$, respectively, where $M_{i n j}$ is the mean mass released by a single SN explosion, that is, $16 \mathrm{M}_{\odot}$ for the SNe II and $1 \mathrm{M}_{\odot}$ for the SNe I.

Finally, we assume that the supermassive black hole (SMBH) in the center injects an AGN wind with a total constant luminosity of $\sim 10^{42} \mathrm{erg} \mathrm{s}^{-1}$ in a cylindrical volume of $14 \mathrm{pc}^{3}$ above and below the midplane of the galaxy characterized by a nearly nonrelativistic velocity perpendicular to the plane of the disk of $2.1 \times 10^{9} \mathrm{~cm} \mathrm{~s}^{-1}$ and with a total rate of injected matter of $\sim 6 \times 10^{-3} \mathrm{M}_{\odot} \mathrm{yr}^{-1}$. This wind is injected in two different ways, either as a collimated outflow with $0^{\circ}$ opening angle, or with an opening angle of $10^{\circ}$.

In all simulations the initial metallicity at the midplane is assumed to be equal to the solar one $\left(z / z_{\odot}=1\right)^{3}$ and decreases inversely proportional to the height of the disk,

\footnotetext{
${ }^{3}$ In Astronomy, all chemical elements abundances are denoted by $Z$, except for the hydrogen $(X)$ and Helium $(Y)$, being the hydrogen and helium abundance by mass, respectively. The abundance gives the mass fraction of a given chemical element in a system. All chemical elements heavier than $\mathrm{H}$ and $\mathrm{He}$ (the metals) are produced in stellar interiors only. Supernovae (SNe) are a major source of metals. In SN II, nuclear fusion during the explosion is responsible for many elements of the $\alpha$-type, like Oxygen and Silicon. On the other hand, SN Ia are the dominant producers of iron (Fe). Typically, one measures just one metallicity, for example the Fe abundance that is often the easiest to measure, and then assumes that the other elements scale with Fe. For the Sun, the total amount of metals by mass is about $z_{\odot}=0.02$, which means that 2 per cent of the mass in the solar system is not hydrogen or helium. For other stars, one usually compares the metallicity in units of the solar value on a logarithmic scale
} 


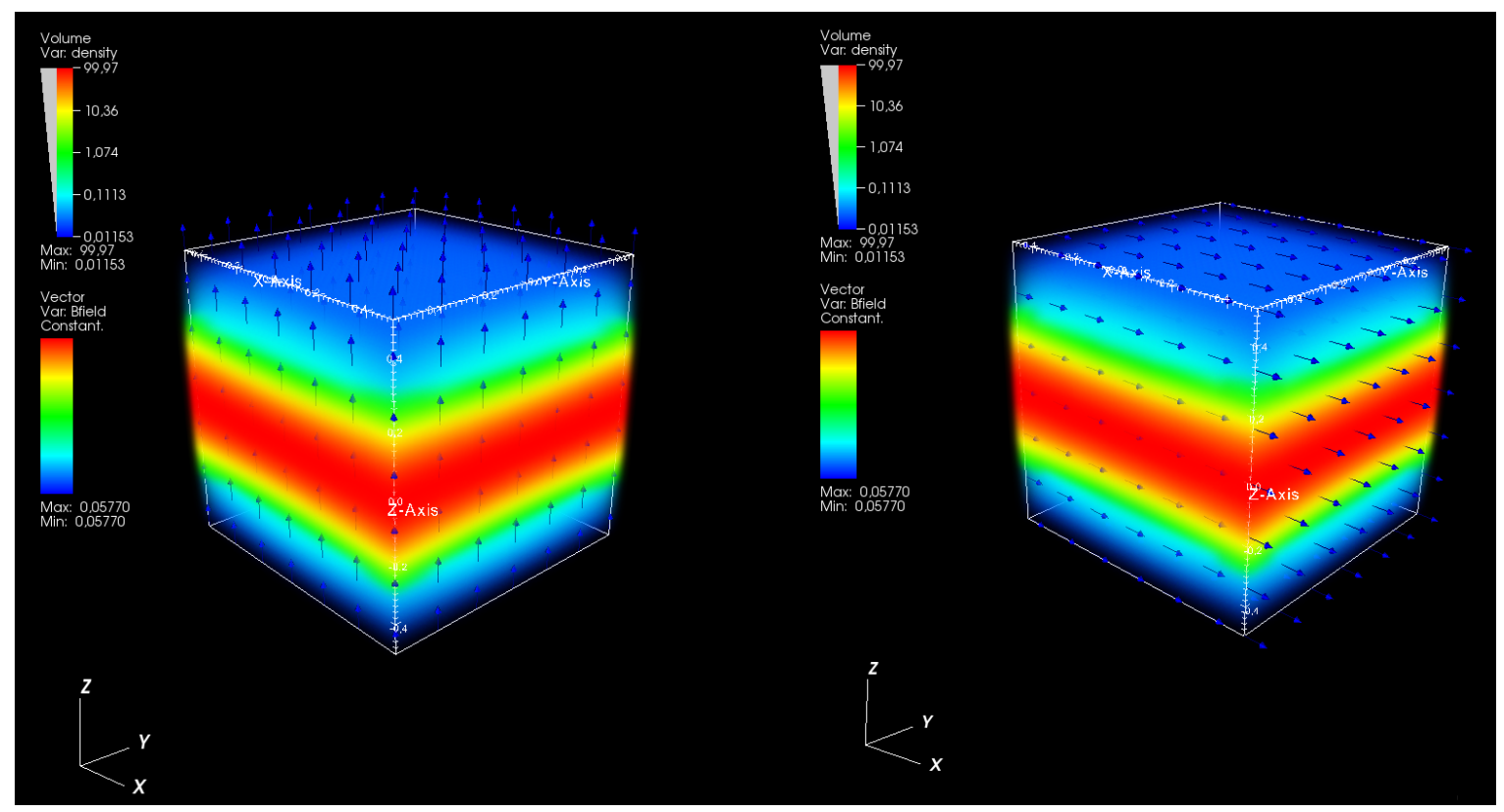

Figure 2.3: Edge-on logarithmic gas density distribution (with density given in $\mathrm{cm}^{-3}$ ) for the central region of the galaxy within $1 \mathrm{kpc}^{3}$, with the arrows representing initial configurations of the magnetic field adopted in this work. . On the left $\vec{B}_{z}$ and on the right $\vec{B}_{x}$. Distances are given in pc.

up to a minimum value $z_{\text {min }} / z_{\odot}=0.1$. We note that the metallicity can influence the value of the cooling function up to a factor of 10 especially at gas temperatures $\sim 10^{5}$ $\mathrm{K}$, but it is not as important for the gas cooling at temperatures between $10^{4}$ and $5 \times 10^{4}$ K, or above $10^{7} \mathrm{~K}$ (Melioli and de Gouveia Dal Pino (2015); Melioli et al. (2013), and references therein). Since the explosions of supernovae are able to inject heavy metals into the interestellar medium of the galaxy, therefore increasing its metallicity, in order to account for this increase in our simulations we have considered a similar prescription as in Melioli et al. (2013, see also references therein).

The setup conditions above will be employed both in HD and MHD simulation models. In the case of the MHD-models, we introduce a uniform magnetic field with two diferent initial geometries, one is parallel and the other is perpendicular to the disk of the galaxy. We consider two different initial uniform magnetic field intensities of $0.76 \mu \mathrm{G}$ and $2.40 \mu \mathrm{G}$ corresponding to a thermal pressure to magnetic pressure ratio of $\beta=p /\left(B_{0}^{2} / 8 \pi\right)=300$ and $\beta=30$, respectively, where $B_{0}$ is the initial intensity of the magnetic field. The initial thermal pressure is $\rho_{o} c_{s, o}^{2}=6.90 \times 10^{-12} \mathrm{erg} \mathrm{cm}^{-3}$, where $c_{s, o}=33 \mathrm{~km} \mathrm{~s}^{-1}$ is the initial sound speed in the midplane of the disk.

When present, the magnetic field is introduced in the initial setup, as shown in Figure 2.3. Due to the large values of $\beta$, the strength of the magnetic field does not affect much $\overline{[\mathrm{Fe} / \mathrm{H}]=\log _{10}\left[(\mathrm{Fe} / \mathrm{H}) /(\mathrm{Fe} / \mathrm{H})_{\odot}\right]=\log _{10}}\left[z / z_{\odot}\right]$. So, if a star have $[\mathrm{Fe} / \mathrm{H}]=0$, that means that it have the same metallicity than the Sun. 
Table 2.2: Initial conditions adopted in the HD and MHD Simulations for 11 Different Models

\begin{tabular}{|c|c|c|c|c|c|c|c|}
\hline Model & $\begin{array}{l}\text { SN Ia } \\
\left(y r^{-1}\right)\end{array}$ & $\begin{array}{c}\text { SFR } \\
\left(\mathrm{M}_{\odot} \mathrm{yr}^{-1}\right)\end{array}$ & $\begin{array}{c}\text { AGN-wind } \\
\text { power } \\
\left(\mathrm{erg} \mathrm{s}^{-1}\right)\end{array}$ & $\begin{array}{c}\text { AGN-wind } \\
\text { opening angle }\end{array}$ & $\begin{array}{c}\mathrm{B}_{x} \\
(\mu \mathrm{G})\end{array}$ & $\begin{array}{c}\mathrm{B}_{z} \\
(\mu \mathrm{G})\end{array}$ & $\beta$ \\
\hline HD-SN Ia-SN II & 0.01 & 1 & - & - & - & - & $\infty$ \\
\hline HD-SN Ia-SN II-AGN $0^{\circ}$ & 0.01 & 1 & $10^{42}$ & $0^{\circ}$ & - & - & $\infty$ \\
\hline HD-SN Ia-SN II-AGN $10^{\circ}$ & 0.01 & 1 & $10^{42}$ & $10^{\circ}$ & - & - & $\infty$ \\
\hline MHD-SN Ia-SN II-B $x_{x}-\beta_{300}$ & 0.01 & 1 & - & - & 0.76 & - & 300 \\
\hline MHD-SN Ia-SN II-B $z-\beta_{300}$ & 0.01 & 1 & - & - & - & 0.76 & 300 \\
\hline MHD-SN Ia-SN II-AGN $0^{\circ}-B_{x}-\beta_{300}$ & 0.01 & 1 & $10^{42}$ & $0^{\circ}$ & 0.76 & - & 300 \\
\hline MHD-SN Ia-SN II-AGN $0^{\circ}-B_{z}-\beta_{300}$ & 0.01 & 1 & $10^{42}$ & $0^{\circ}$ & - & 0.76 & 300 \\
\hline MHD-SN Ia-SN II-AGN $10^{\circ}-\mathrm{B}_{x}-\beta_{300}$ & 0.01 & 1 & $10^{42}$ & $10^{\circ}$ & 0.76 & - & 300 \\
\hline MHD-SN Ia-SN II-AGN $10^{\circ}-B_{z}-\beta_{300}$ & 0.01 & 1 & $10^{42}$ & $10^{\circ}$ & - & 0.76 & 300 \\
\hline MHD-SN Ia-SN II-AGN $0^{\circ}-\mathrm{B}_{x}-\beta_{30}$ & 0.01 & 1 & $10^{42}$ & $0^{\circ}$ & 2.40 & - & 30 \\
\hline MHD-SN Ia-SN II-AGN $10^{\circ}-B_{x}-\beta_{30}$ & 0.01 & 1 & $10^{42}$ & $10^{\circ}$ & 2.40 & - & 30 \\
\hline
\end{tabular}

the initial pressure and density stratification of the system. As the system starts to evolve, these quantities rapidly adjust themselves only very slightly in order to establish a new hydrostatic equilibrium in the rotating disk.

All the models simulated in this work and their initial conditions are presented in Table 2.2. In the next Chapter, we present the results of the numerical simulations of these models. 

CHAPTER 3

\section{Results OF HD AND MHD Numerical Simulations}

In this chapter, we describe the results of the HD and MHD simulations performed for a set of eleven models sweeping the parametric space described in Table 2.2. We compare these models and present an analysis of the effects of the feedback by the SF and the SMBH-driven winds on the central regions of the host galaxy and the environment.

Figures 3.1 to 3.19 present 2D central cuts of the distributions of density, velocity, temperature, pressure, thermal pressure to magnetic pressure ratio $(\beta)$ and square of the magnetic field strength (in the case of MHD models) within the one-kpc region of Seyferts-like galaxies at four snapshots: 0.225, 0.75, 3.0 and 5.0 Myr for different models of Table 2.2. In the following sections, we describe the main results of these models.

\subsection{HD-Models}

\subsubsection{Feedback from SN Ia and SN II sources (HD-SN Ia-SN II)}

In this section, we describe the hydrodynamical model named "HD-SN Ia-SN II" of Table 2.2, which considers only star-formation-driven outflows and no AGN wind, i.e., with energy driving sources due to the SN Ia and SN II explosions in the halo and the disk.

Figure 3.1 shows the evolution of this model. At $t=0.225 \mathrm{Myr}$, we clearly see the 
initial formation of little bubbles due to the explosions of SN II in the disk and SN Ia in the halo. At $t=0.75 \mathrm{Myr}$, we identify a few denser and colder clumpy structures at high altitudes. The bubbles gradually expand supersonically heating the ISM from $10^{4}$ to $10^{5} \mathrm{~K}$ especially at the midplane of the dense disk. At this stage, the initial density and temperature stratification of the ISM has been destroyed and the gas is fully mixed. At $t=3 \mathrm{Myr}$, the filamentary structures have been swept by the SF-wind, which grows continuously powered by the SN explosions in the disk, transporting cold dense structures from the disk to the halo at $\sim 0.2 \mathrm{kpc}$ altitudes. Almost all the region occupied by the dense disk has reached a mean temperature of $\sim 10^{5} \mathrm{~K}$ due principally to the shock generated by SN II explosions. Finally, at $t=5 \mathrm{Myr}$, the SF-wind has reached the 0.5 kpc levels, with velocities of $\sim 10^{2} \mathrm{~km} \mathrm{~s}^{-1}$ and densities of $10 \mathrm{~cm}^{-3}$.

\subsubsection{HD Model with a collimated AGN-wind and SN Ia and SN II (HD-SN Ia-SN II-AGN $0^{\circ}$ )}

In this model (Figure 3.2) we consider the outflow generated by energy injection by both the SNe and by the AGN with a collimated wind coming out from the center of the galaxy.

Initially, the dynamics of the central region of the system is governed by the AGN outflow because the time-scale it takes to propagate over a $0.5 \mathrm{kpc}$ distance is of the order of $t=0.247 \mathrm{Myr}$, while in the same period only a few SNe have exploded and released a small quantity of energy and mass to the ISM. Earlier, at $t=0.225 \mathrm{Myr}$, we clearly see the formation a bow shock at the collimated wind head when it breaks supersonically the outer boundaries of the thick disk. At this period the bow shock propagation velocity is $\sim 6000 \mathrm{~km} \mathrm{~s}^{-1}$ and it compresses a thin layer of gas with $\sim 30 \mathrm{~cm}^{-3}$. The interaction of the collimated AGN wind with the disk opens a channel around the central region of the galaxy with a maximum diameter of $\sim 80 \mathrm{pc}$ at $t \sim 0.320$ Myr.

At $t=0.75 \mathrm{Myr}$, after leaving the dense disk region, the jet propagates through the halo, producing an outflow that ranges in velocities between $\sim 10^{2}-10^{3} \mathrm{~km} \mathrm{~s}^{-1}$, densities between $10^{-1}-10^{-2} \mathrm{~cm}^{-3}$ and temperatures of $10^{6}-10^{7} \mathrm{~K}$. At this stage, the supersonic shock generated by the AGN-wind, which propagates parallel to the disk with a velocity of $\sim 200 \mathrm{~km} \mathrm{~s}^{-1}$, heats the gas surrounding the jet from $\sim 10^{4} \mathrm{~K}$ to $\sim 5 \times 10^{5} \mathrm{~K}$. From this time on, a comparison with Figure 3.1 shows that the collimated AGN outflow causes but little influence on the large scale evolution of the ISM of the galaxy. It digs a narrow channel along which propagates, sweeping just a small amount of the ISM gas, which is expanding in a broader slower wind by the action of the SNe-driven wind. In this case, the AGN feedback is mainly on the intergalactic medium, well above the computation domain. Similar results were found in Melioli and de Gouveia Dal Pino (2015).

Around $t \sim 3 \mathrm{Myr}$, at the highest altitudes (up and down the disk), where the expand- 
ing AGN wind is pushing out SF-driven wind material, we can see the presence of some cold, high speed, fragmented structures, which are absent in the model with no jet of Figure 3.1 (at the same time step). These clumpy estructures at $\sim 0.4 \mathrm{kpc}$ have densities of a few $10 \mathrm{~cm}^{-3}$, velocities of $\sim 150 \mathrm{~km} \mathrm{~s}^{-1}$ and temperatures of a few $10^{3} \mathrm{~K}$.

We note that these velocities and densities are too low, and the temperatures too high to explain, for instance, the observed features in the molecular outflows, as described in 1.1 , for which (see more below) the observed structures range in velocities of $600-10^{4}$ $\mathrm{km} \mathrm{s}^{-1}$ and densities of $10^{2}-10^{3} \mathrm{~cm}^{-3}$ (Morganti et al. (2013), Tadhunter et al. (2014))

\subsubsection{HD-model with SF wind and AGN wind with $10^{\circ}$ opening angle (HD-SN Ia-SN II-AGN 10 ${ }^{\circ}$ )}

Distinct from the previous model, in this case, we injected an AGN-wind with an opening angle of $10^{\circ}$ above and below the central region of the galactic disk. The AGN wind propagates until it reaches the boundary of the domain at $0.255 \mathrm{Myr}$, later than in model HD-SN Ia-SN II-AGN $0^{\circ}$, because of its larger cross-section which makes it sweep much more surrounding gas than in the collimated wind model. At this stage the AGNwind has broken the disk, removing from the core region of the galaxy about $99 \%$ of gas available for accretion onto the SMBH. As the AGN-wind propagates supersonically, a surface discontinuity separates the AGN outflow from the disk ambient gas. The AGN wind is strongly collimated by the confining ambient pressure (see the larger pressure at the interface around the AGN-wind at $t=0225 \mathrm{Myr}$ ). Our simulations reveal that whenever new SN II explosions occur in the disk, this causes the growth of the ambient pressure and thus the increase in the collimation. As the AGN outflow propagates, we identify re-collimation shocks along its extension, as usually detected in jet simulations.

The bow shock at the end of the jet propagates with almost the same velocity $(\sim$ $6000 \mathrm{~km} \mathrm{~s}^{-1}$ ) as in the previous model, sweeping a thin layer of gas with almost the same density and temperature.

This time, the channel opened by the AGN-wind is $20 \mathrm{pc}$ bigger than in the previous model, reaching a maximum diameter $\sim 100 \mathrm{pc}$ around the central source at $t=0.352$ Myr.

At $t=0.75 \mathrm{Myr}$, the AGN-wind has exceeded the $1 \mathrm{kpc}$ scales forming an outflow similar to the previous model, but larger, ranging in velocities between $\sim 10^{2}$ and $10^{3} \mathrm{~km}$ $\mathrm{s}^{-1}$, densities of $10^{-1}-10^{-3} \mathrm{~cm}^{-3}$ and temperatures of $10^{6}-10^{7} \mathrm{~K}$.

As in the previous model, some clumpy fragmented structures form at the interacting region between the $\mathrm{AGN}$-wind and the disk gas at $t \sim 0.75 \mathrm{Myr}$. Later, at $t=3 \mathrm{Myr}$, these same structures are located at $\mathrm{z} \sim 0.4 \mathrm{kpc}$ above and below the galactic disk, with densities of a few $10^{-1}-1 \mathrm{~cm}^{-3}$, velocities of $\sim 100 \mathrm{~km} \mathrm{~s}^{-1}$ and temperatures between $10^{3}-10^{4} \mathrm{~K}$. As in the case of the collimated AGN-wind model, this model at this stage 
is also unable to drive the observed structures in molecular outflows.

After $t \sim 3.0 \mathrm{Myr}$, the SF-driven wind fully develops, as in the previous model. The shocks produced by the SNe heat and blow the gas located in the denser disk, increasing the thermal pressure that both drives the SF-wind expansion and helps to confine the AGN-wind channel. The SN explosions heat the gas and transport it to levels of $\sim 200$ pc above and below the disk, where the radiative cooling is not as effective as in the central region. When this heated gas escapes to the halo, it enriches it with ionized material coming from the inner regions of the galaxy. This dynamic produces a slightly stratified SF-wind in density and temperature, with densities between $1-10 \mathrm{~cm}^{-3}$ and temperatures of $10^{4} \mathrm{~K}$ for the more expanded gas, and $3.1 \times 10^{4} \mathrm{~K}$ for the denser. Clearly, as in the previous model, the SF wind and the AGN wind are separated by a surface discontinuity that keeps the two flows in pressure equilibrium. On one hand, the AGNwind, flowing almost vertically, reaches velocities as high as of $2 \times 10^{4} \mathrm{~km} \mathrm{~s}^{-1}$, while the SF-wind occupies almost the entire domain and has velocities as high as a few $100 \mathrm{~km}$ $\mathrm{S}^{-1}$.

The overall comparison between Figures 3.2 and 3.3 indicates that the macroscopic evolution of the system is very similar to that of the collimated AGN wind model of Figure 3.2, only that the larger opening angle allows for a larger interaction with the surrounding ISM and the SF-wind, as much more gas can be pushed by the AGN wind. As in the collimated AGN-wind, the density and amount of gas transported by the AGNwind is clearly much smaller than that of the expanding gas driven by the SNe-driven wind. A remarkable feature in this model that is more pronounced than in the model of Figure 3.1 is the development of a fountain-like structure at the highest altitudes when the AGN outflow scapes from the disk. This makes part of the expanding gas in the SF-wind to fallback into the galaxy, and the decrease of the mass loss-rate in the outflow (as we will see in Section 3.3.3 below). This fallback process is also observed and provides a 'positive' feedback on the host galaxy evolution (e.g., Melioli et al. (2008); Combes (2018)). 
HD-SNI-SNII
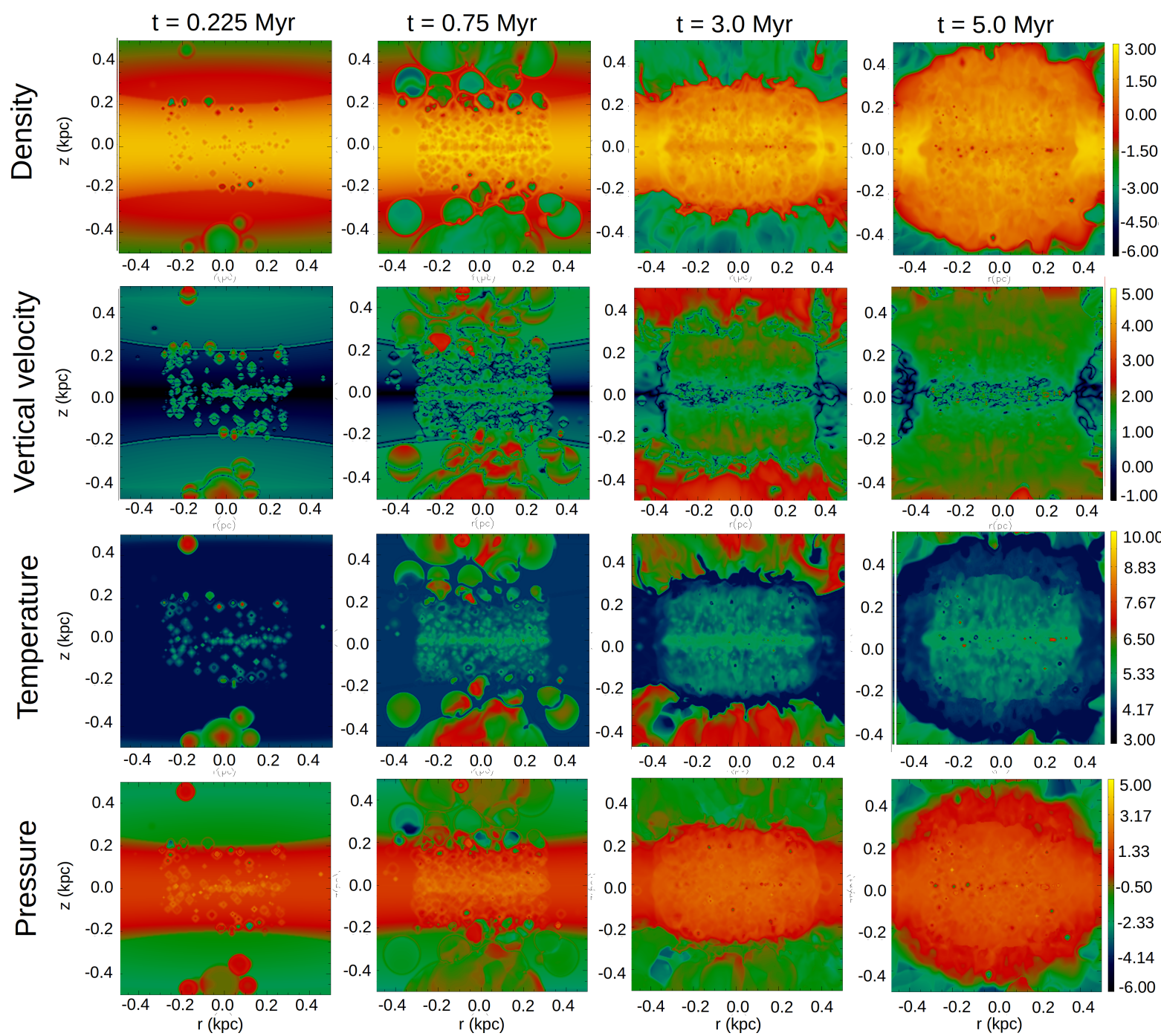

Figure 3.1: Edge-on logarithmic 2D cuts of the central slice of the model with SF-driven outflow and no AGN-wind, named HD-SN Ia-SN II (See also 2.2). Density is in $\mathrm{cm}^{-3}$ (top), vertical velocity in $\mathrm{km} \mathrm{s}^{-1}$ (top middle), temperature in $\mathrm{K}$ (bottom middle) and pressure in units of $7 \times 10^{-12} \mathrm{erg} \mathrm{cm}^{-3}$ )(bottom) for four different times of evolution: $t=0.225,0.75,3.0$ and 5.0 Myr (from left to right respectively). 


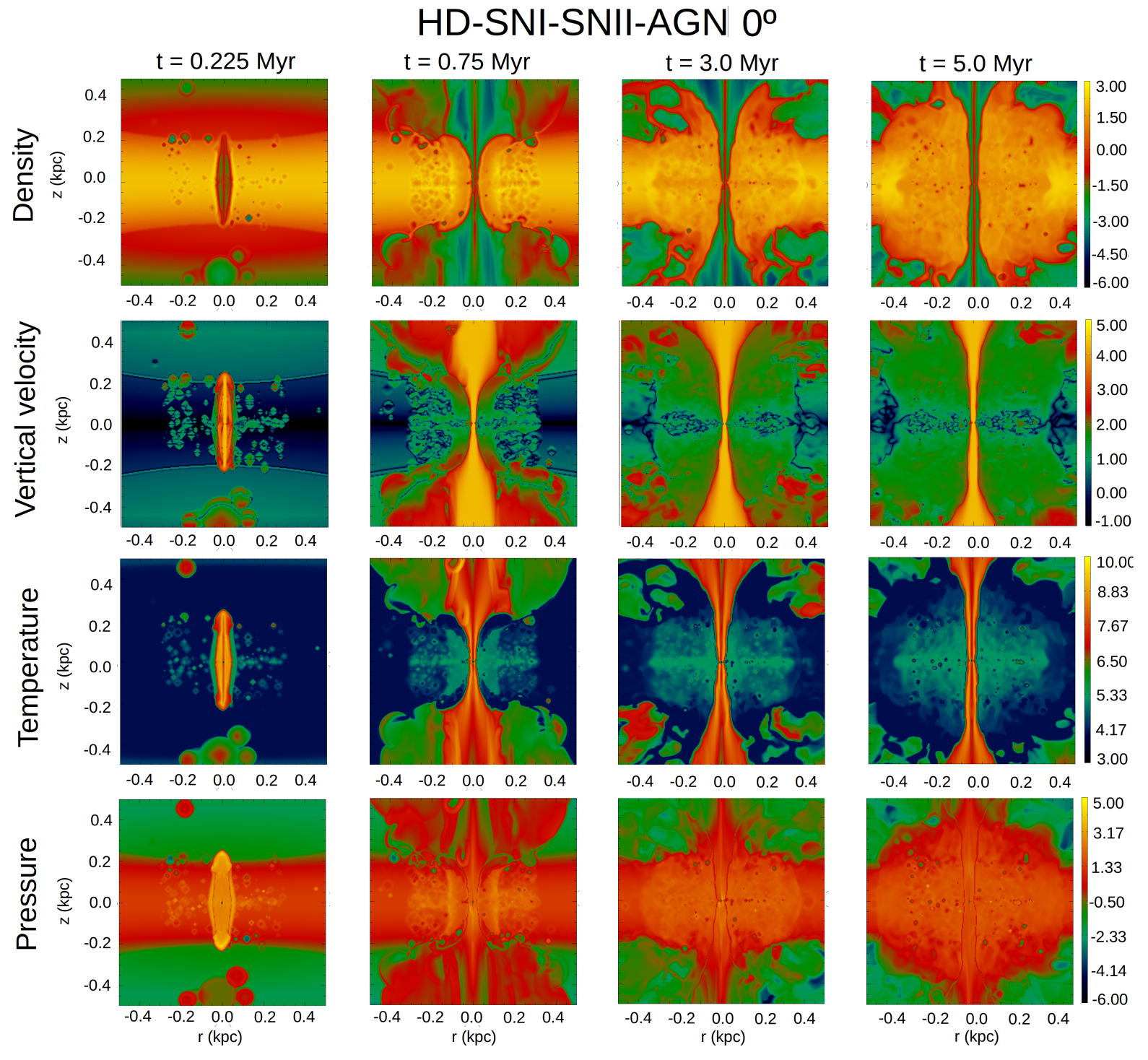

Figure 3.2: Edge-on logarithmic 2D cuts of the central slice of the model with SF-driven outflow and a collimated AGN-wind, named HD-SN Ia-SN II-AGN 0 (See also 2.2). Density is in $\mathrm{cm}^{-3}$ (top), vertical velocity in $\mathrm{km} \mathrm{s}^{-1}$ (top middle), temperature in $\mathrm{K}$ (bottom middle) and pressure in units of $7 \times 10^{-12} \mathrm{erg} \mathrm{cm}^{-3}$ )(bottom) for four different times of evolution: $t=0.225$, $0.75,3.0$ and 5.0 Myr (from left to right respectively). 


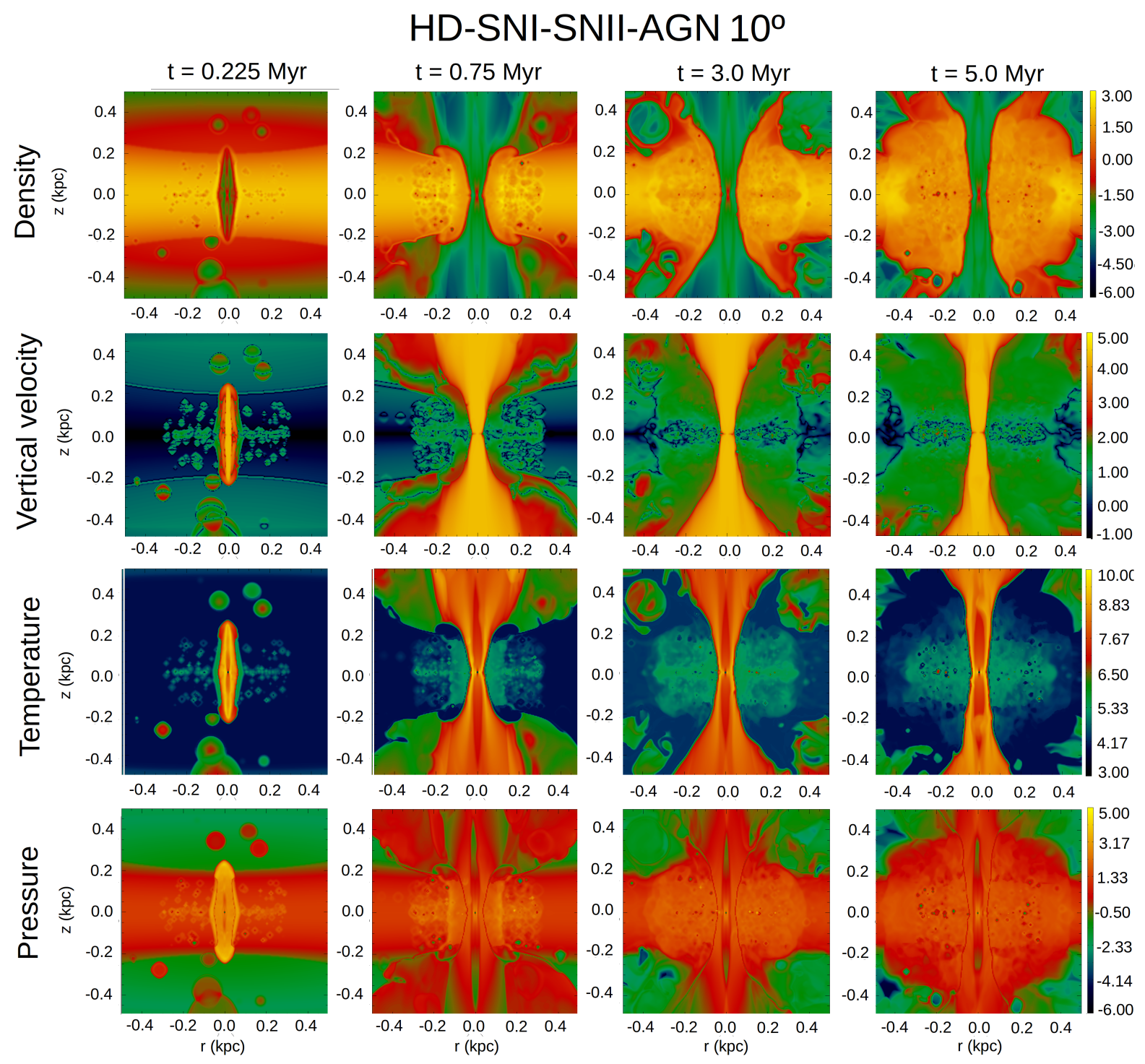

Figure 3.3: Edge-on logarithmic 2D cuts of the central slice of the model with SF-driven outflow and a no-collimated AGN-wind ( $10^{\circ}$ opening angle), named HD-SN Ia-SN II-AGN $\mathbf{1 0}^{\circ}$. Density is in $\mathrm{cm}^{-3}$ (top), vertical velocity in $\mathrm{km} \mathrm{s}^{-1}$ (top middle), temperature in $\mathrm{K}$ (bottom middle) and pressure in units of $7 \times 10^{-12} \mathrm{erg} \mathrm{cm}^{-3}$ )(bottom) for four different times of evolution: $t=0.225,0.75,3.0$ and $5.0 \mathrm{Myr}$ (from left to right respectively). 


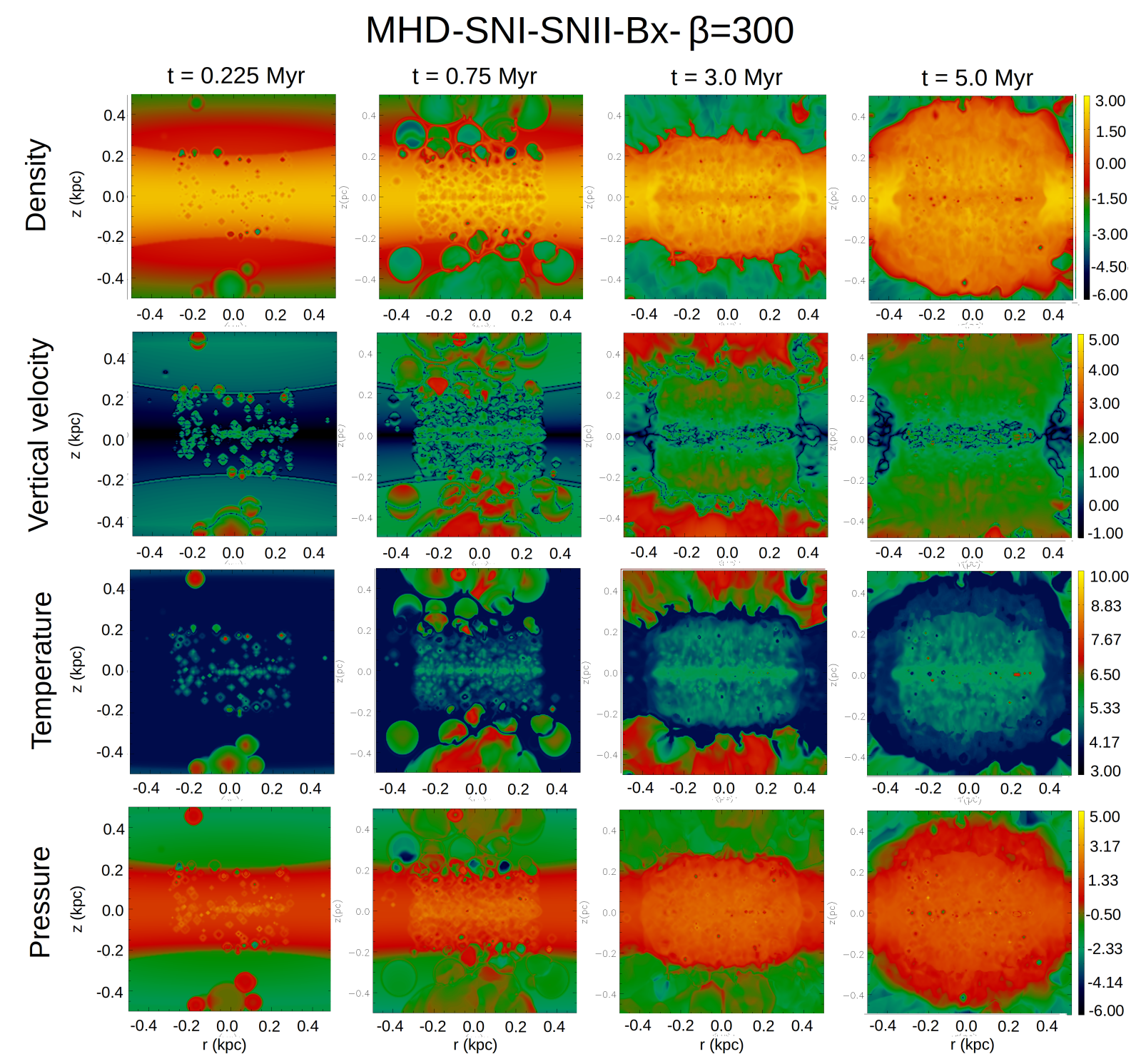

Figure 3.4: Edge-on logarithmic 2D cuts of the central slice of the model with SF-driven outflow, no AGN-wind and an inital horizontal magnetic field, named MHD-SN Ia-SN II$B_{x}-\beta_{300}$. Density is in $\mathrm{cm}^{-3}$ (top), vertical velocity in $\mathrm{km} \mathrm{s}^{-1}$ (top middle), temperature in $\mathrm{K}$ (bottom middle) and pressure in units of $7 \times 10^{-12} \mathrm{erg} \mathrm{cm}^{-3}$ )(bottom) for four different times of evolution: $t=0.225,0.75,3.0$ and $5.0 \mathrm{Myr}$ (from left to right respectively). 


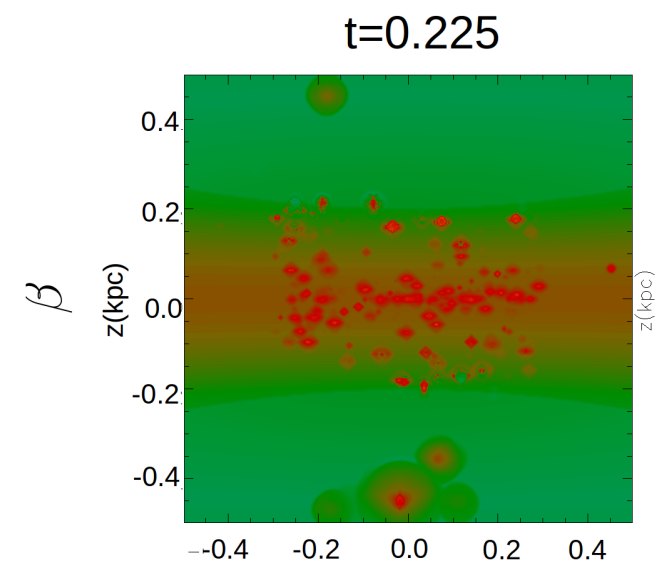

\section{MHD-SNI-SNII-Bx- $\beta=300$}
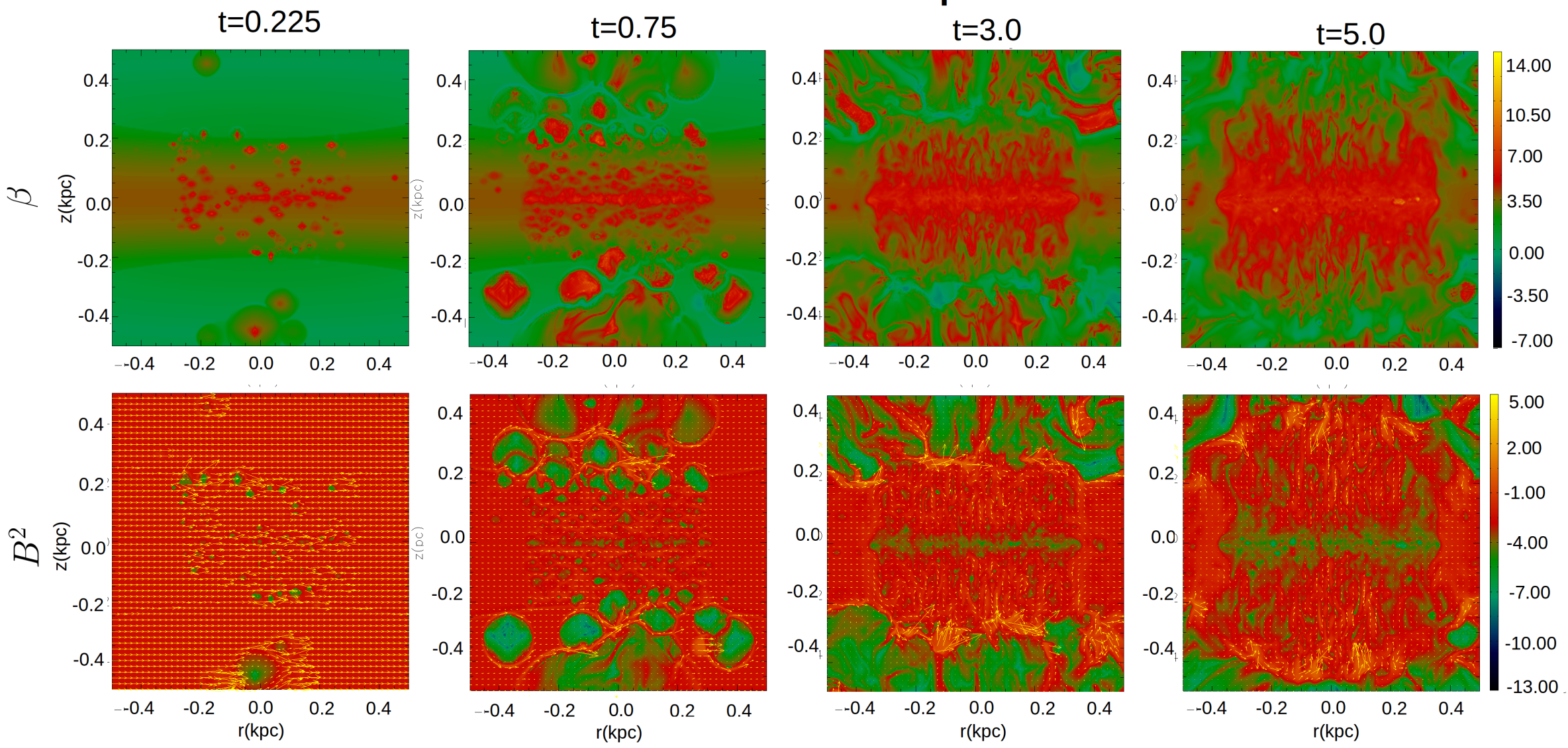

Figure 3.5: Edge-on logarithmic 2D cuts of the central slice of the model with SF-driven outflow, no AGN-wind and an inital horizontal magnetic field, named MHD-SN Ia-SN II- $B_{x}-\beta_{300}$ for four diferent times of evolution: $t=0.225,0.75,3.0$ and 5.0 Myr (from left to right respectively). $\beta$ is adimensional (top). $B^{2}$ is in units of $0.76 \mu \mathrm{G}$ (bottom) 


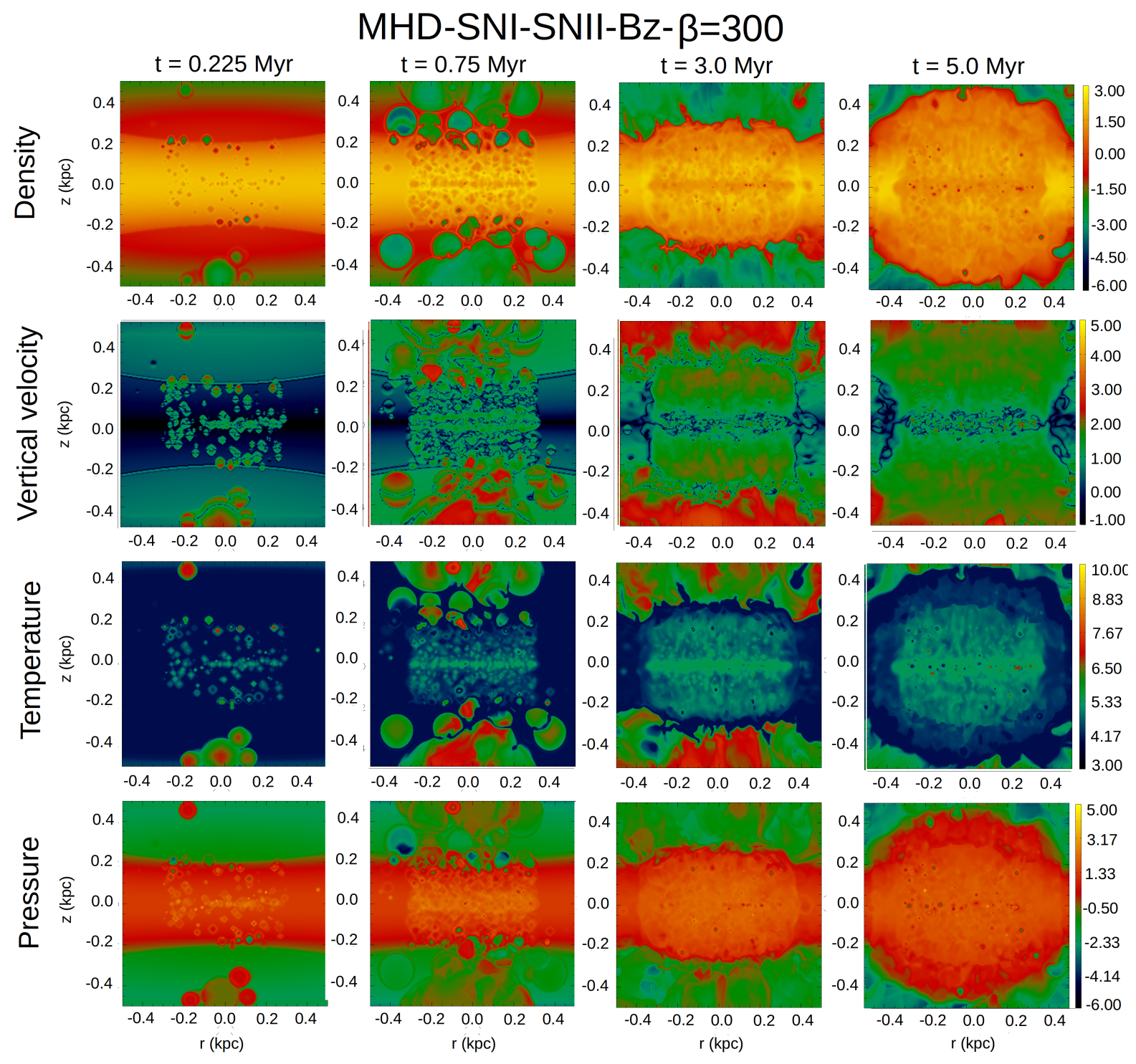

Figure 3.6: Edge-on logarithmic 2D cuts of the central slice of the model with SF-driven outflow, no AGN-wind and an inital vertical magnetic field, named MHD-SN Ia-SN II- $B_{z^{-}}$ $\beta_{300}$. Density is in $\mathrm{cm}^{-3}$ (top), vertical velocity in $\mathrm{km} \mathrm{s}^{-1}$ (top middle), temperature in $\mathrm{K}$ (bottom middle) and pressure in units of $7 \times 10^{-12} \mathrm{erg} \mathrm{cm}^{-3}$ )(bottom) for four different times of evolution: $t=0.225,0.75,3.0$ and $5.0 \mathrm{Myr}$ (from left to right respectively). 


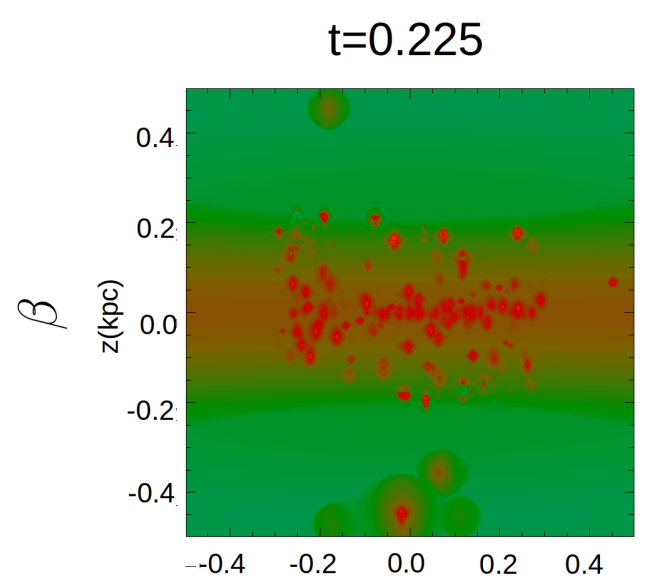

\section{MHD-SNI-SNII-BZ- $\beta=300$}
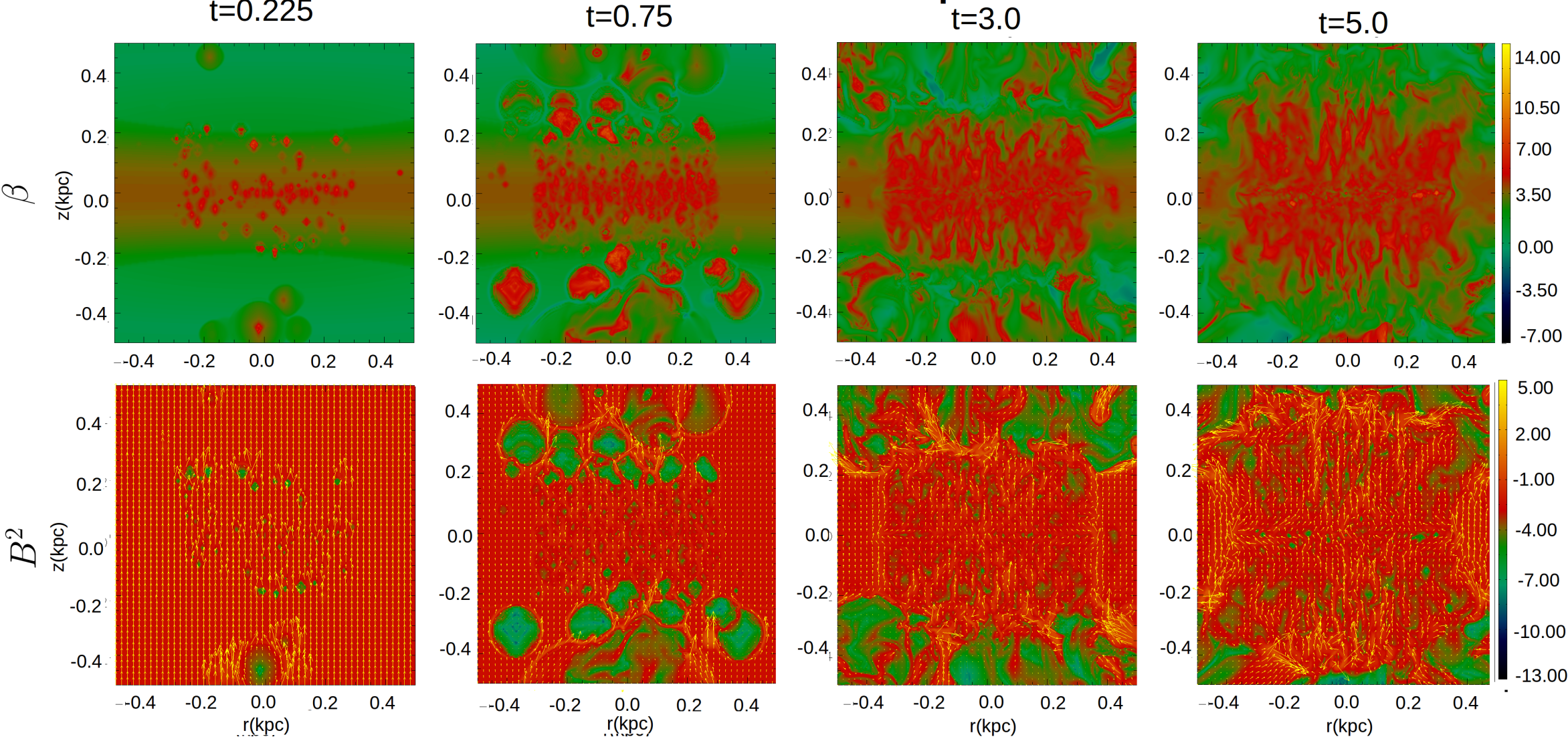

Figure 3.7: Edge-on logarithmic 2D cuts of the central slice of the model with SF-driven outflow, no AGN-wind and an inital vertical magnetic field, named MHD-SN Ia-SN II- $B_{z}-\beta_{300}$ for four diferent times of evolution: $t=0.225,0.75,3.0,5.0$ Myr (from left to right respectively). $\beta$ is adimensional (top). $B^{2}$ is in units of $0.76 \mu \mathrm{G}$ (bottom) 


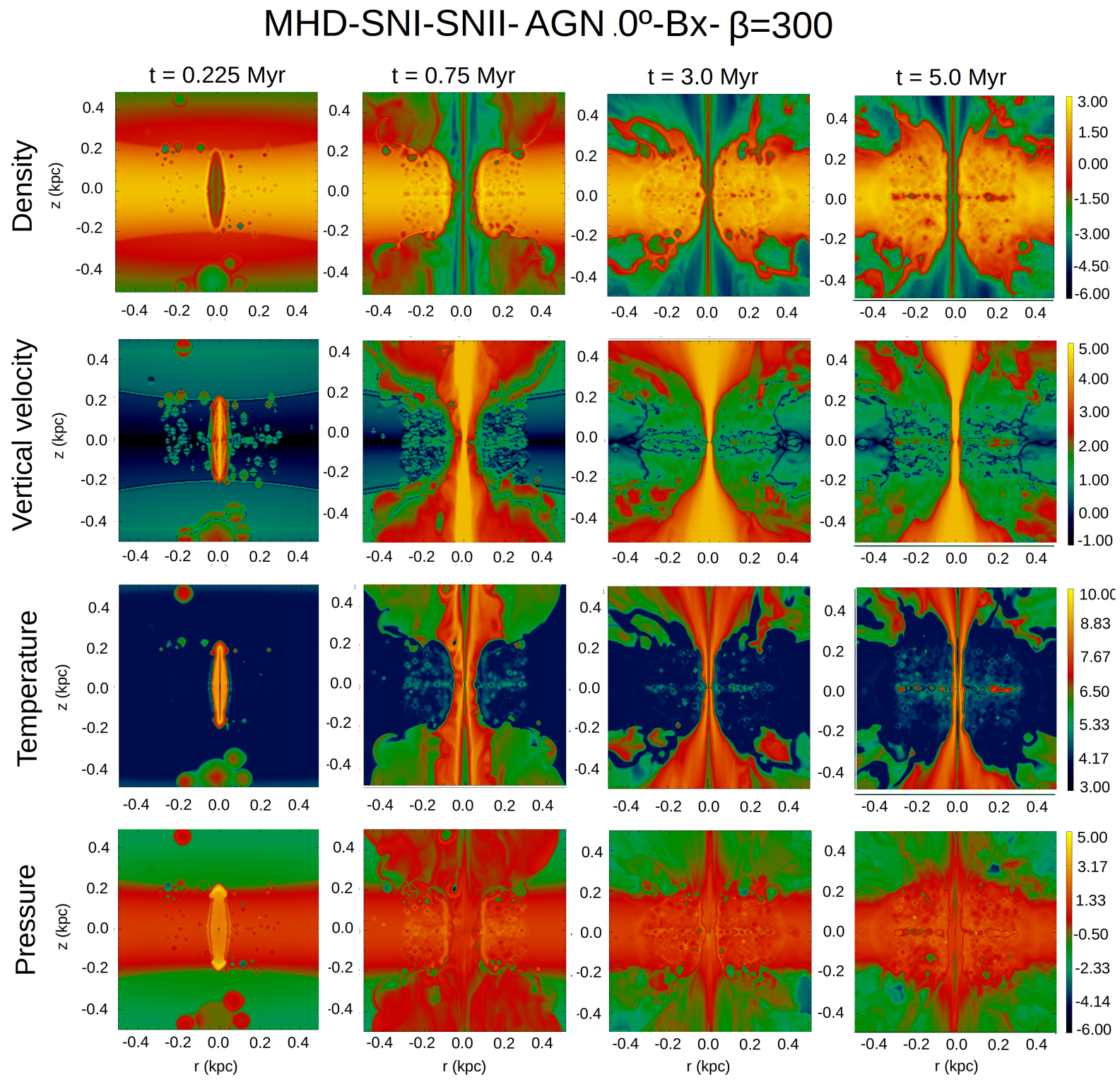

Figure 3.8: Edge-on logarithmic 2D cuts of the central slice of the model with SF-driven outflow, a collimated AGN-wind $\left(0^{\circ}\right.$ opening angle) and an inital horizontal magnetic field, named MHD-SN Ia-SN II-AGN $0^{\circ}-B_{x}-\beta_{300}$. Density is in $\mathrm{cm}^{-3}$ (top), vertical velocity in $\mathrm{km} \mathrm{s}^{-1}$ (top middle), temperature in $\mathrm{K}$ (bottom middle) and pressure in units of $7 \times 10^{-12} \mathrm{erg}$ $\mathrm{cm}^{-3}$ )(bottom) for four different times of evolution: $t=0.225,0.75,3.0$ and $5.0 \mathrm{Myr}$ (from left to right respectively). 


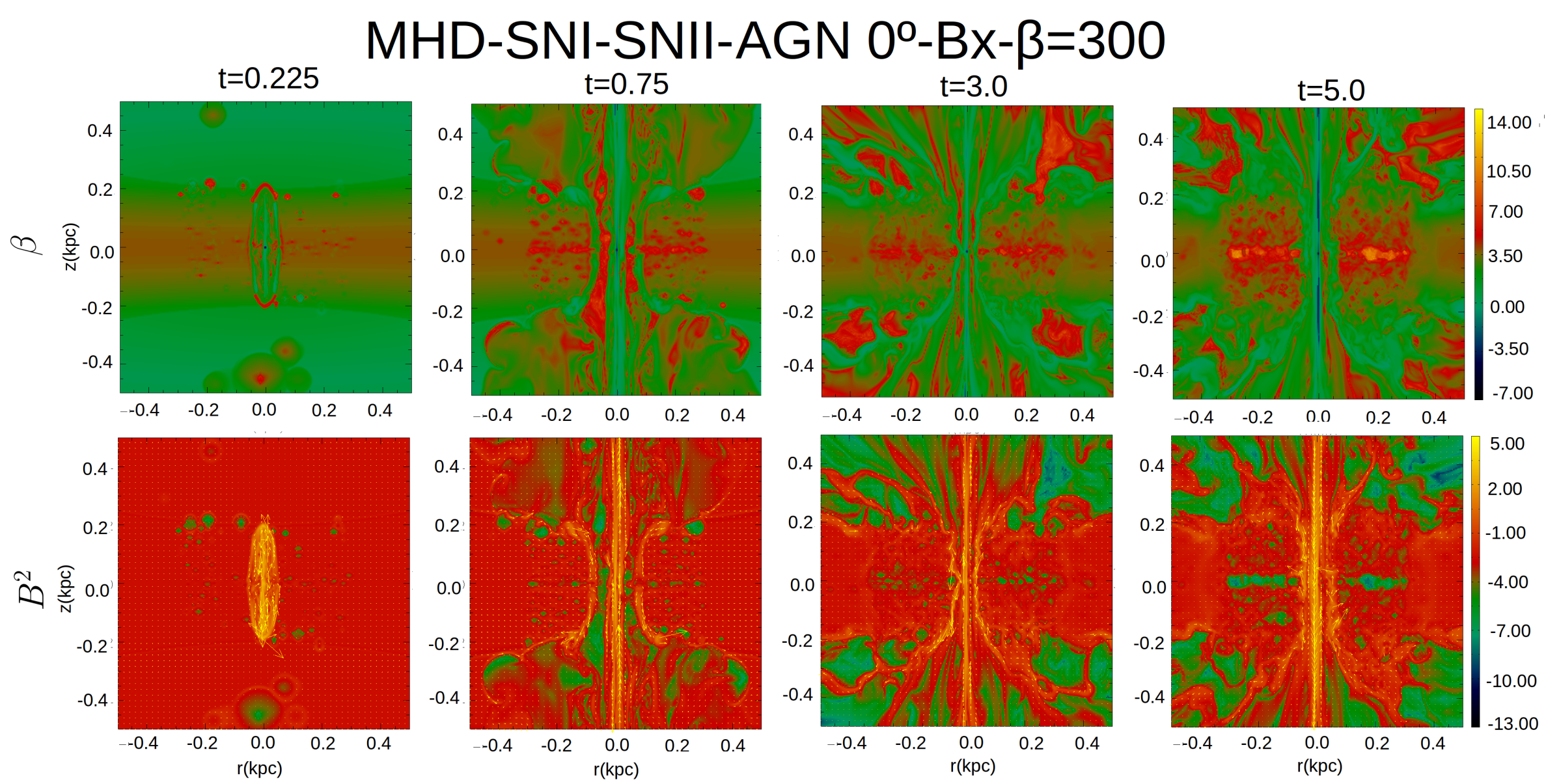

Figure 3.9: Edge-on logarithmic $2 \mathrm{D}$ cuts of the central slice of the model with SF-driven outflow, collimated AGN-wind $\left(0^{\circ}\right.$ opening angle) and an inital horizontal magnetic field, named MHD-SN Ia-SN II-AGN $0^{\circ}-B_{x}-\beta_{300}$ for four diferent times of evolution: $t=0.225,0.75,3.0,5.0$ Myr (from left to right respectively). $\beta$ is adimensional (top). $B^{2}$ is in units of $0.76 \mu \mathrm{G}$ (bottom) 


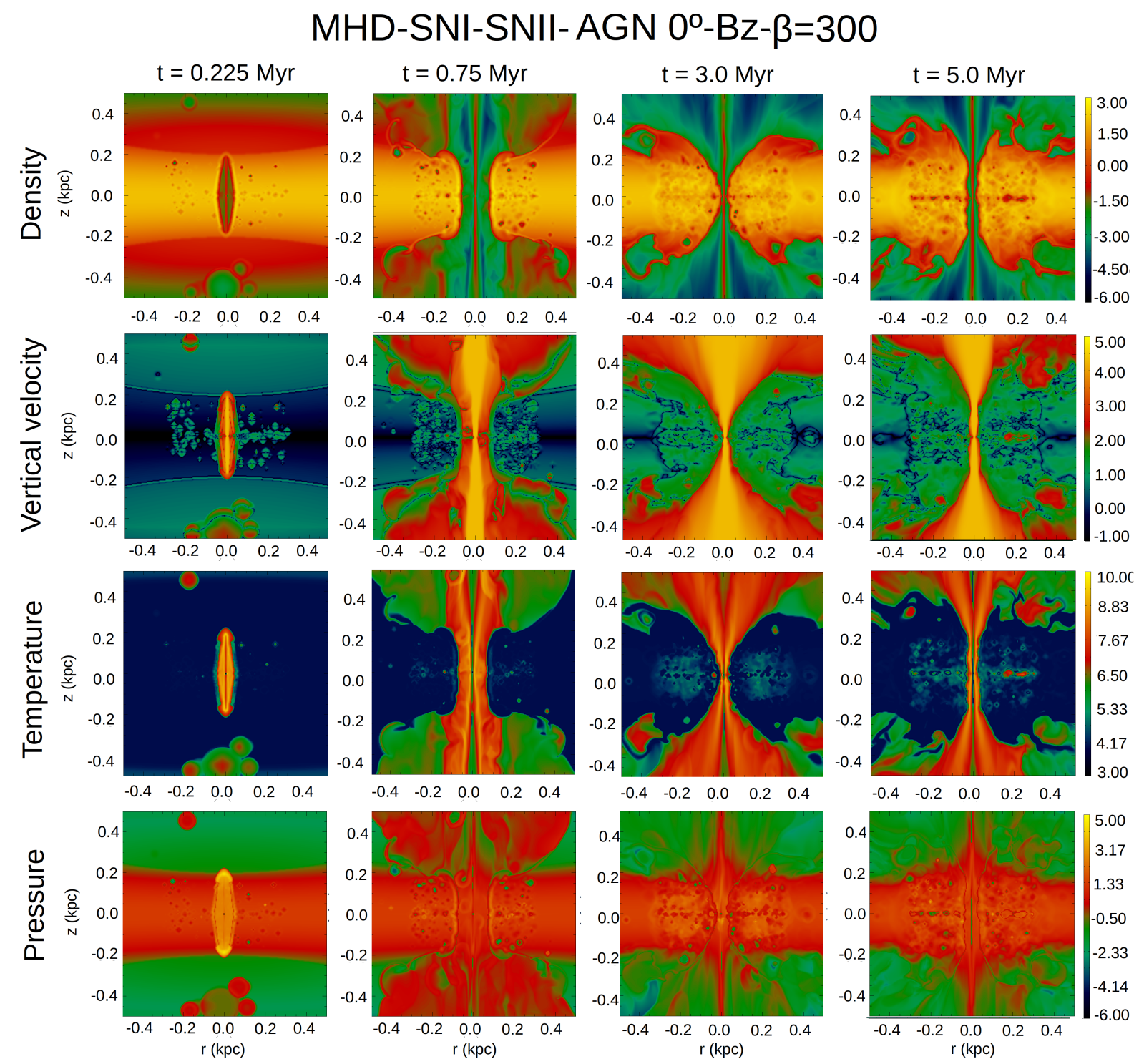

Figure 3.10: Edge-on logarithmic 2D cuts of the central slice of the model with SF-driven outflow, a collimated AGN-wind ( $0^{\circ}$ opening angle) and an inital vertical magnetic field, named MHD-SN Ia-SN II-AGN $0^{\circ}-B_{z}-\beta_{300}$. Density is in $\mathrm{cm}^{-3}$ (top), vertical velocity in $\mathrm{km}$ $\mathrm{s}^{-1}$ (top middle), temperature in $\mathrm{K}$ (bottom middle) and pressure in units of $7 \times 10^{-12} \mathrm{erg}$ $\mathrm{cm}^{-3}$ )(bottom) for four different times of evolution: $t=0.225,0.75,3.0,5.0 \mathrm{Myr}$ (from left to right respectively). 


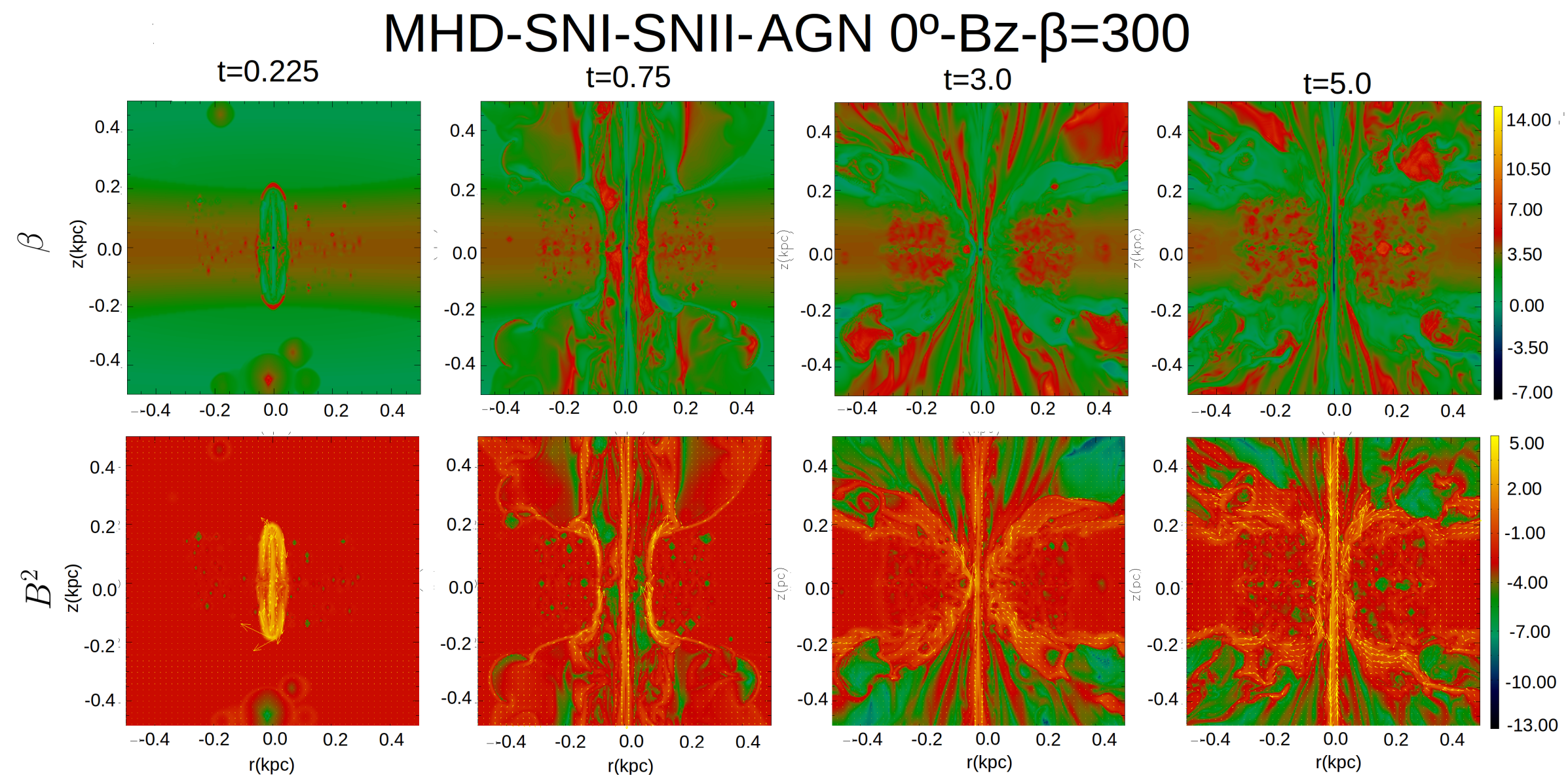

Figure 3.11: Edge-on logarithmic 2D cuts of the central slice of the model with SF-driven outflow, collimated AGN-wind ( $0^{\circ}$ opening angle) and an inital vertical magnetic field, named MHD-SN Ia-SN II-AGN $0^{\circ}-B_{z}-\beta_{300}$ for four diferent times of evolution: $t=0.225,0.75,3.0,5.0$ Myr (from left to right respectively). $\beta$ is adimensional (top). $B^{2}$ is in units of $0.76 \mu \mathrm{G}$ (bottom) 


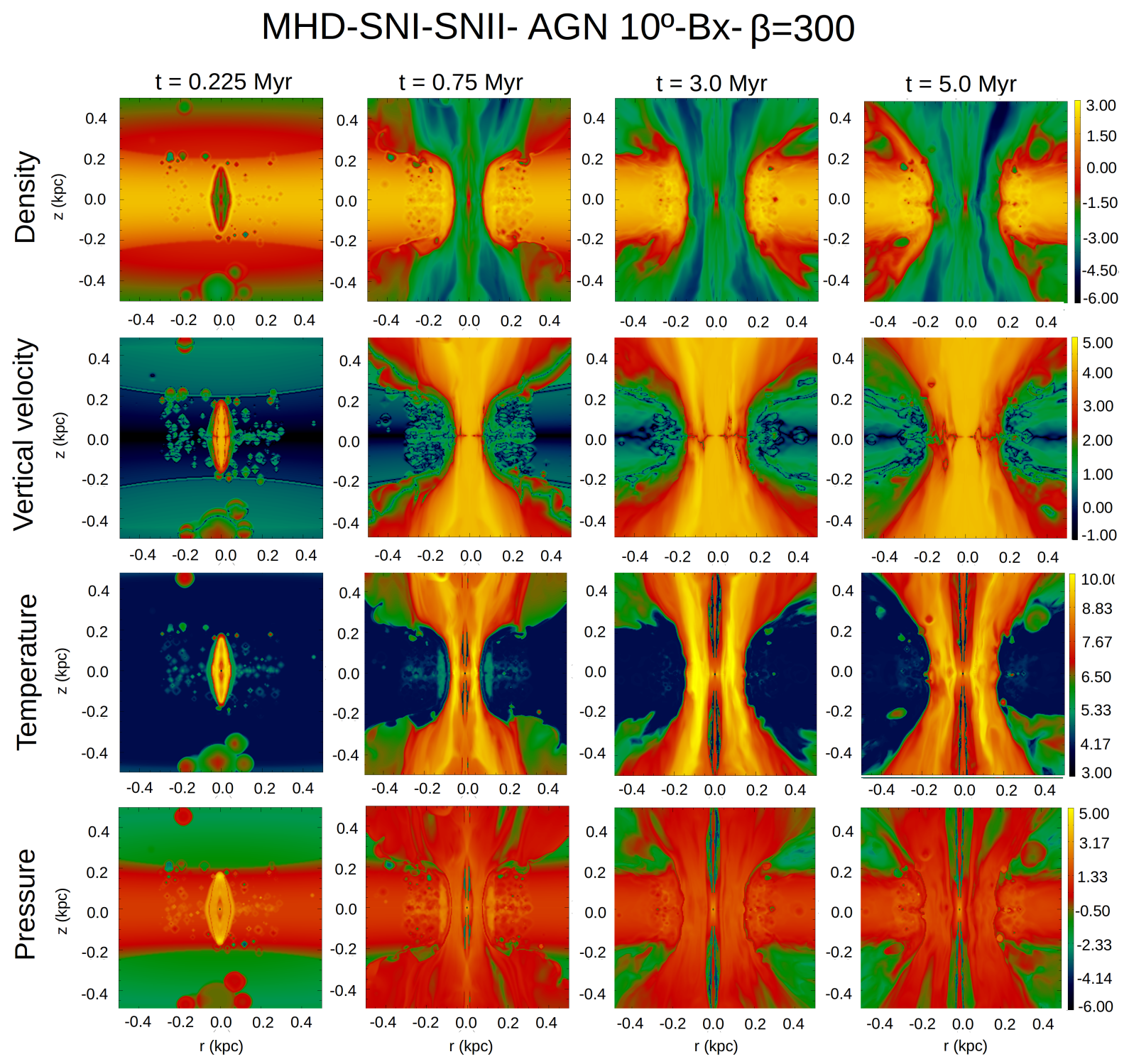

Figure 3.12: Edge-on logarithmic $2 \mathrm{D}$ cuts of the central slice of the model with SF-driven outflow, a no-collimated AGN-wind ( $10^{\circ}$ opening angle) and an inital horizontal magnetic field, named MHD-SN Ia-SN II-AGN $10^{\circ}-B_{x}-\beta_{300}$. Density is in $\mathrm{cm}^{-3}$ (top), vertical velocity in $\mathrm{km} \mathrm{s}^{-1}$ (top middle), temperature in $\mathrm{K}$ (bottom middle) and pressure in units of $7 \times 10^{-12} \mathrm{erg}$ $\mathrm{cm}^{-3}$ )(bottom) for four different times of evolution: $t=0.225,0.75,3.0,5.0 \mathrm{Myr}$ (from left to right respectively). 


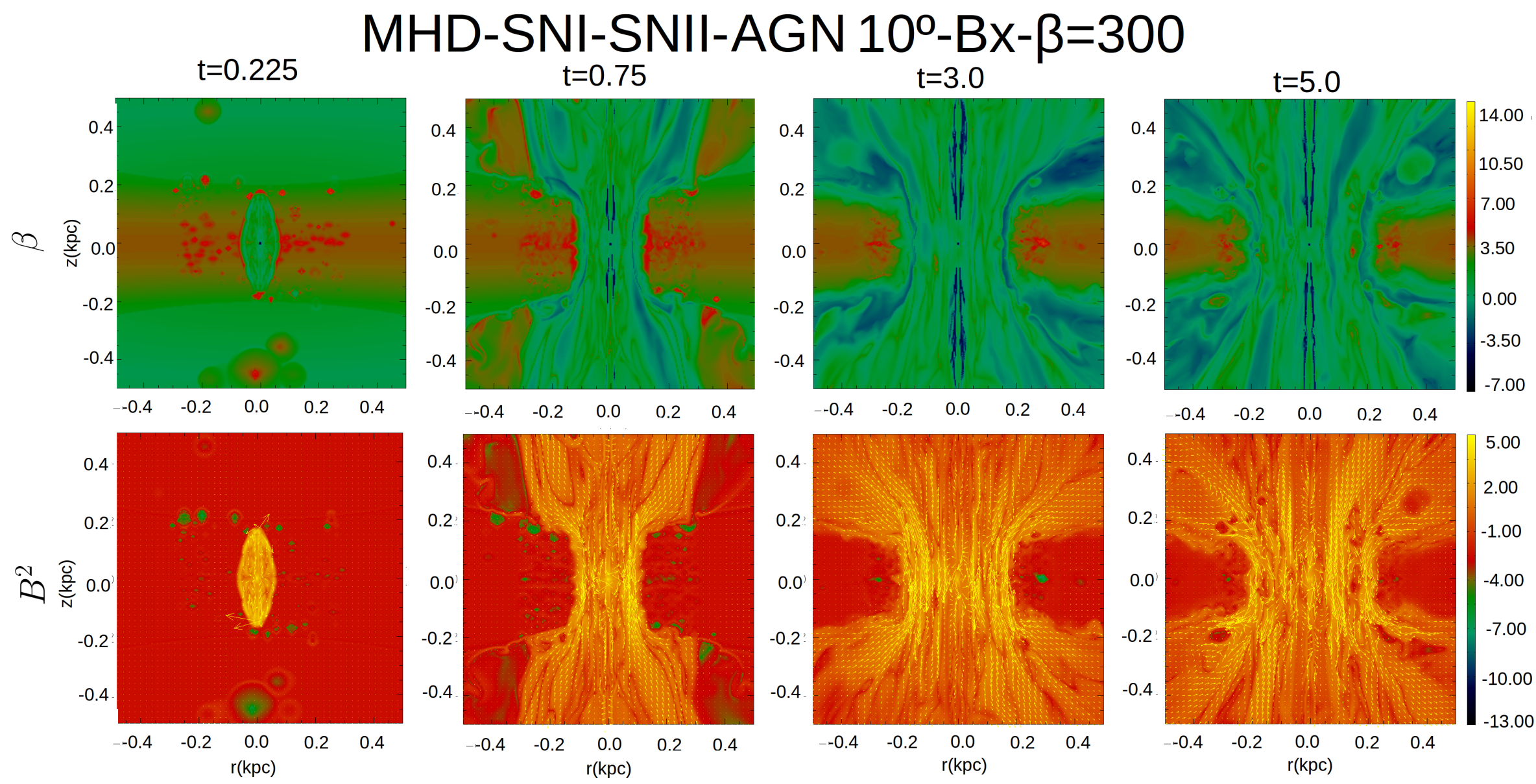

Figure 3.13: Edge-on logarithmic $2 \mathrm{D}$ cuts of the central slice of the model with SF-driven outflow, no-collimated AGN-wind (10 opening angle) and an inital horizontal magnetic field, named MHD-SN Ia-SN II-AGN $10^{\circ}-B_{x}-\beta_{300}$ for four diferent times of evolution: $t=0.225,0.75,3.0,5.0$ $\operatorname{Myr}$ (from left to right respectively). $\beta$ is adimensional (top). $B^{2}$ is in units of $0.76 \mu \mathrm{G}$ (bottom) 


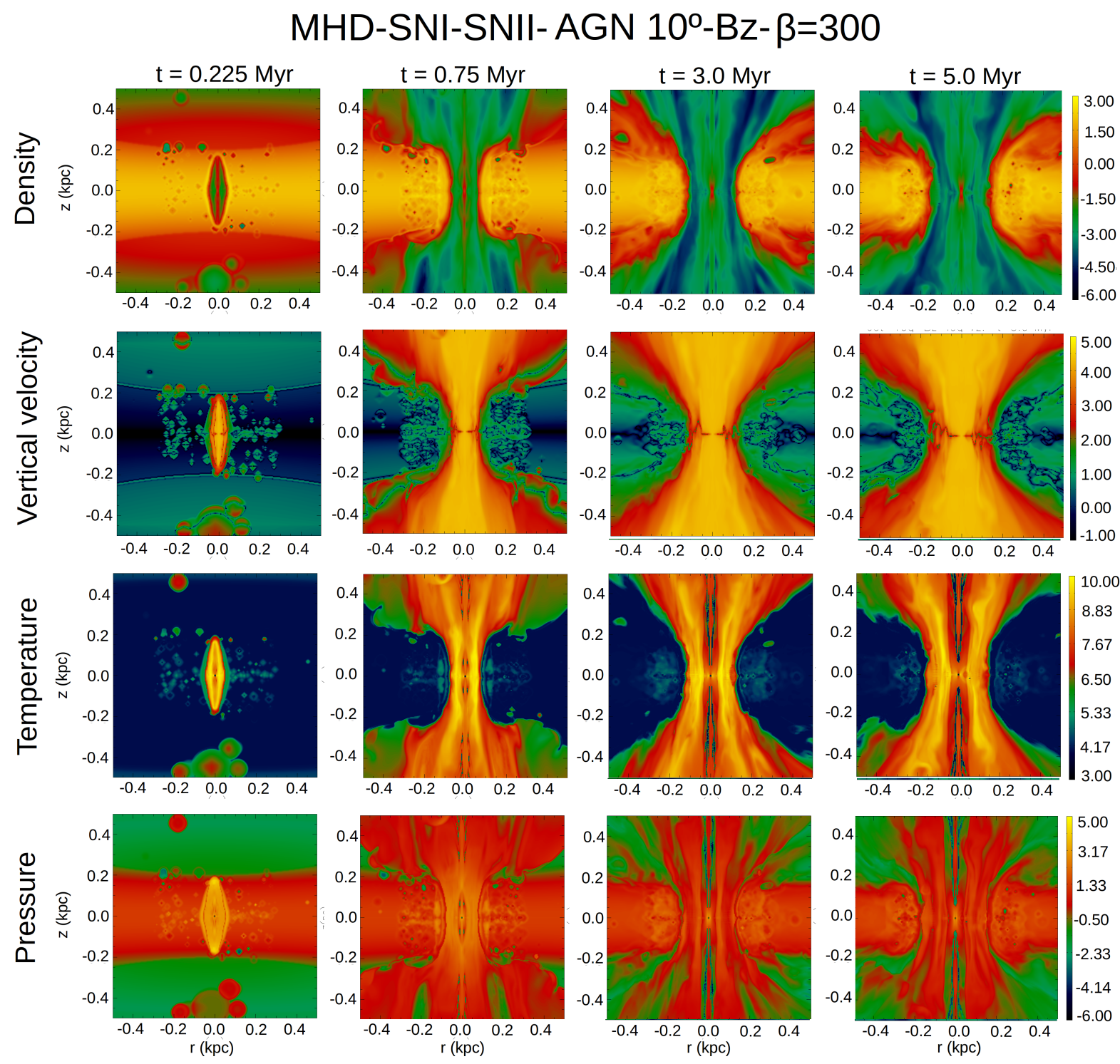

Figure 3.14: Edge-on logarithmic $2 \mathrm{D}$ cuts of the central slice of the model with SF-driven outflow, a no-collimated AGN-wind ( $10^{\circ}$ opening angle) and an inital vertical magnetic field, named MHD-SN Ia-SN II-AGN $10^{\circ}-B_{z}-\beta_{300}$. Density is in $\mathrm{cm}^{-3}$ (top), vertical velocity in $\mathrm{km} \mathrm{s}^{-1}$ (top middle), temperature in $\mathrm{K}$ (bottom middle) and pressure in units of $7 \times 10^{-12} \mathrm{erg}$ $\mathrm{cm}^{-3}$ )(bottom) for four different times of evolution: $t=0.225,0.75,3.0,5.0 \mathrm{Myr}$ (from left to right respectively). 


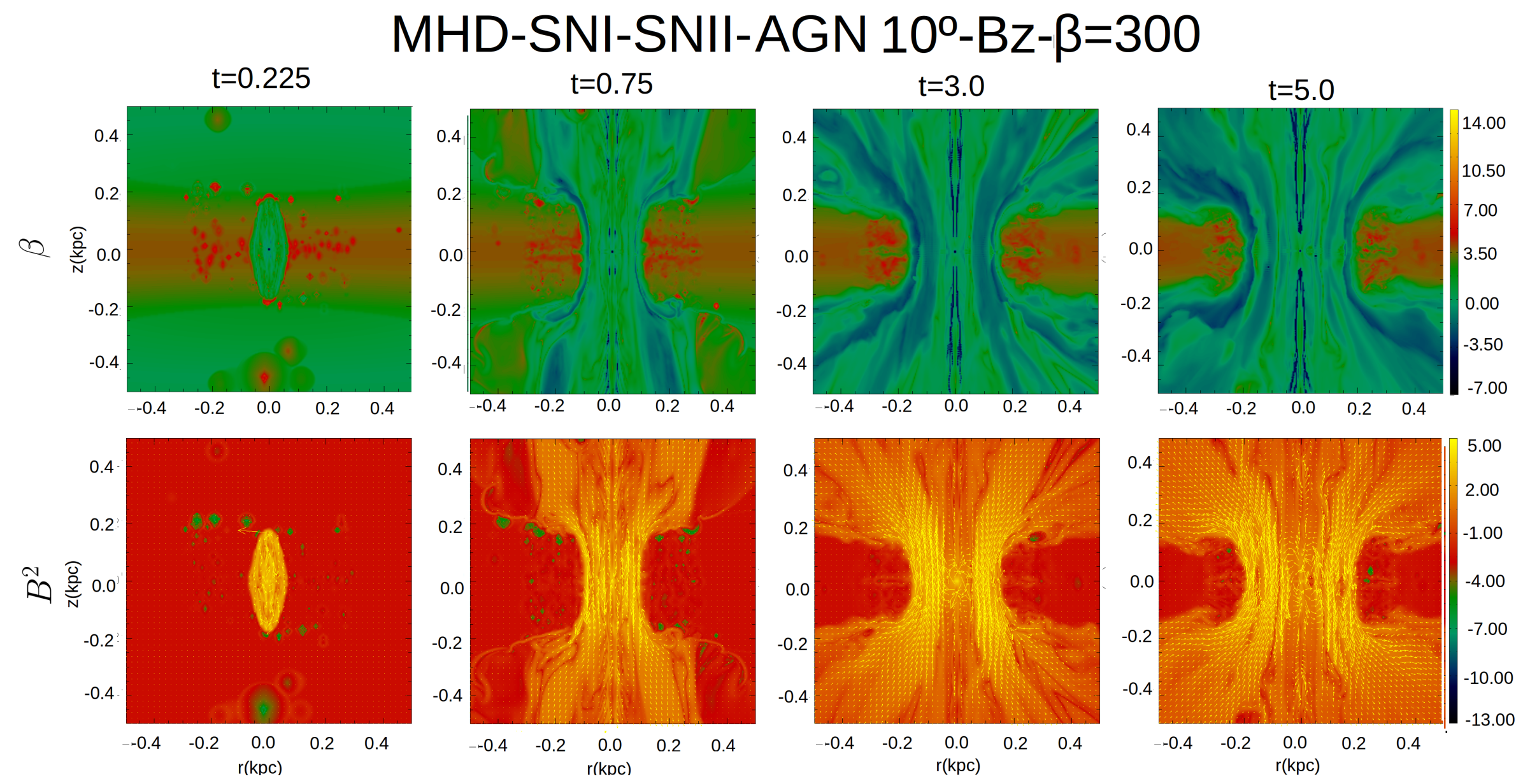

Figure 3.15: Edge-on logarithmic $2 \mathrm{D}$ cuts of the central slice of the model with SF-driven outflow, no-collimated AGN-wind (10 ${ }^{\circ}$ opening angle) and an inital vertical magnetic field, named MHD-SN Ia-SN II-AGN $10^{\circ}-B_{z}-\beta_{300}$ for four diferent times of evolution: $t=0.225,0.75,3.0,5.0$ $\operatorname{Myr}$ (from left to right respectively). $\beta$ is adimensional (top). $B^{2}$ is in units of $0.76 \mu \mathrm{G}$ (bottom). 


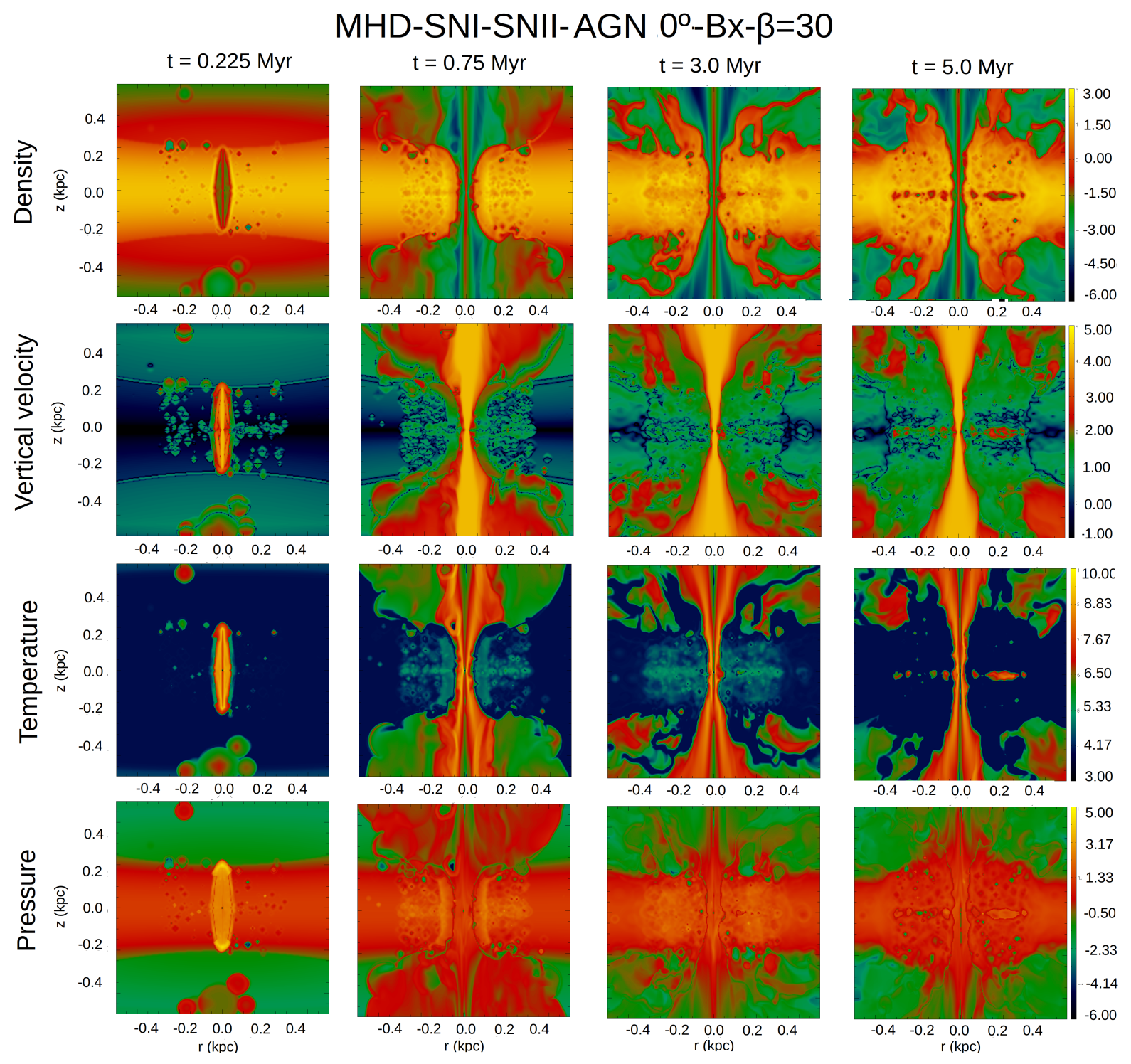

Figure 3.16: Edge-on logarithmic 2D cuts of the central slice of the model with SF-driven o utflow, a collimated AGN-wind ( $0^{\circ}$ opening angle) and an inital horizontal magnetic field, named MHD-SN Ia-SN II-AGN $0^{\circ}-B_{x}-\beta_{30}$. Density is in $\mathrm{cm}^{-3}$ (top), vertical velocity in $\mathrm{km} \mathrm{s}^{-1}$ (top middle), temperature in $\mathrm{K}$ (bottom middle) and pressure in units of $7 \times 10^{-12} \mathrm{erg}$ $\mathrm{cm}^{-3}$ )(bottom) for four different times of evolution: $t=0.225,0.75,3.0,5.0 \mathrm{Myr}$ (from left to right respectively). 


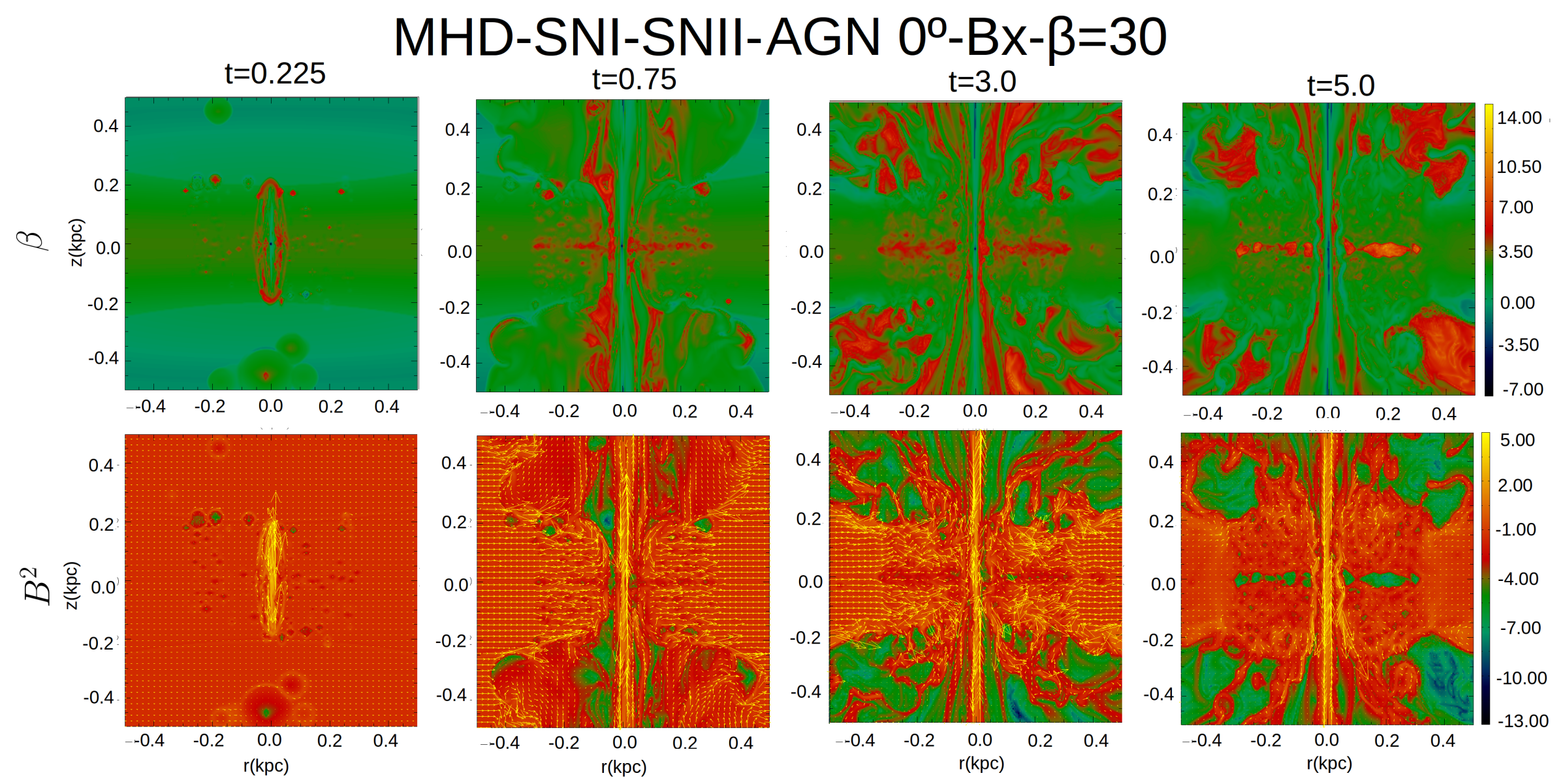

Figure 3.17: Edge-on logarithmic 2D cuts of the central slice of the model with SF-driven outflow, collimated AGN-wind ( $0^{\circ}$ opening angle) and an inital horizontal magnetic field, named MHD-SN Ia-SN II-AGN $0^{\circ}-B_{x^{-}}-\beta=30$ for four diferent times of evolution: $t=0.225,0.75,3.0,5.0$ Myr (from left to right respectively). $\beta$ is adimensional (top). $B^{2}$ is in units of $2.40 \mu \mathrm{G}$ (bottom) 


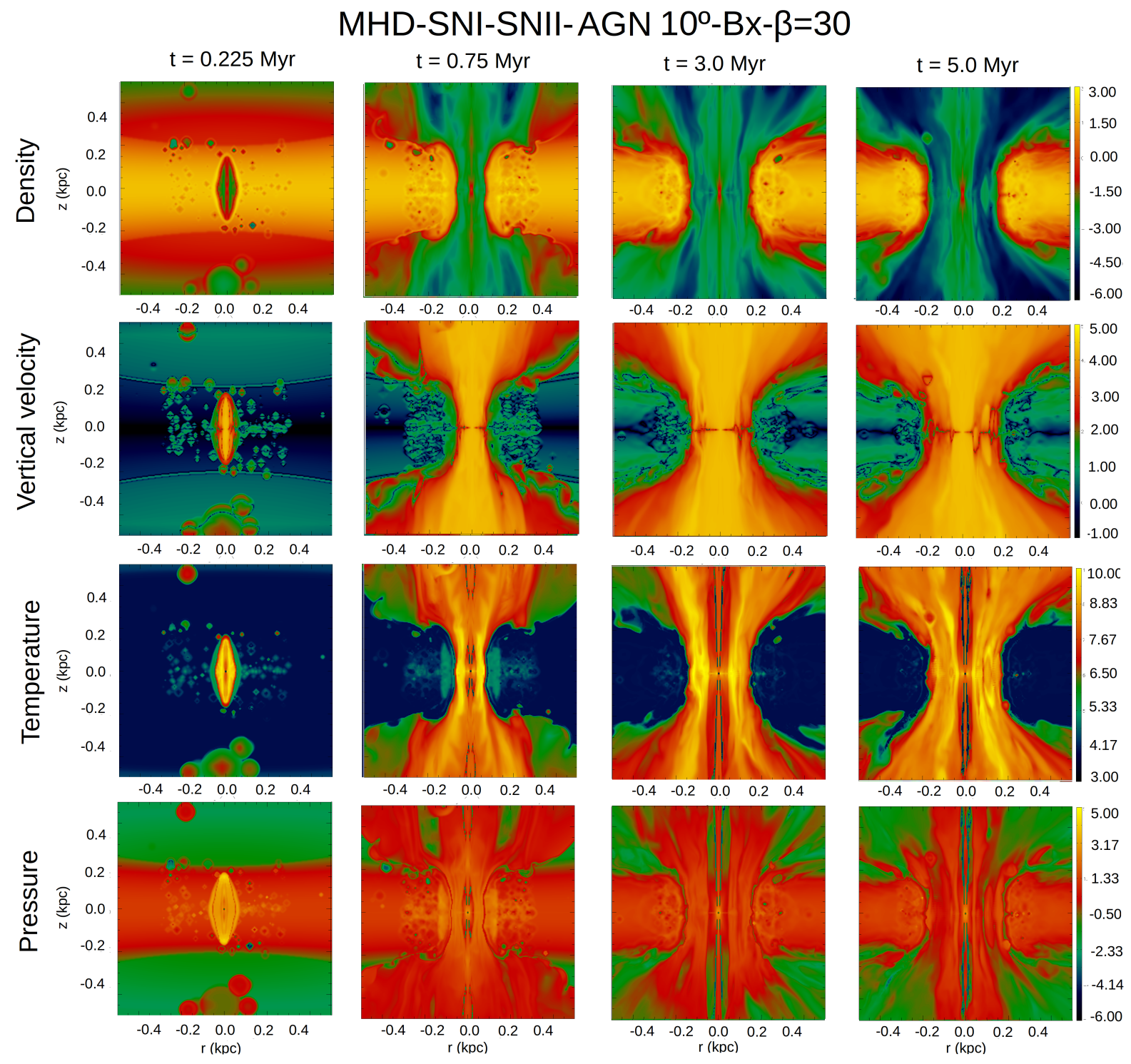

Figure 3.18: Edge-on logarithmic $2 \mathrm{D}$ cuts of the central slice of the model with SF-driven outflow, a no-collimated AGN-wind ( $10^{\circ}$ opening angle) and an inital horizontal magnetic field, named MHD-SN Ia-SN II-AGN $10^{\circ}-B_{x}-\beta_{30}$. Density is in $\mathrm{cm}^{-3}$ (top), vertical velocity in $\mathrm{km} \mathrm{s}^{-1}$ (top middle), temperature in $\mathrm{K}$ (bottom middle) and pressure in units of $7 \times 10^{-12} \mathrm{erg}$ $\mathrm{cm}^{-3}$ )(bottom) for four different times of evolution: $t=0.225,0.75,3.0,5.0 \mathrm{Myr}$ (from left to right respectively). 


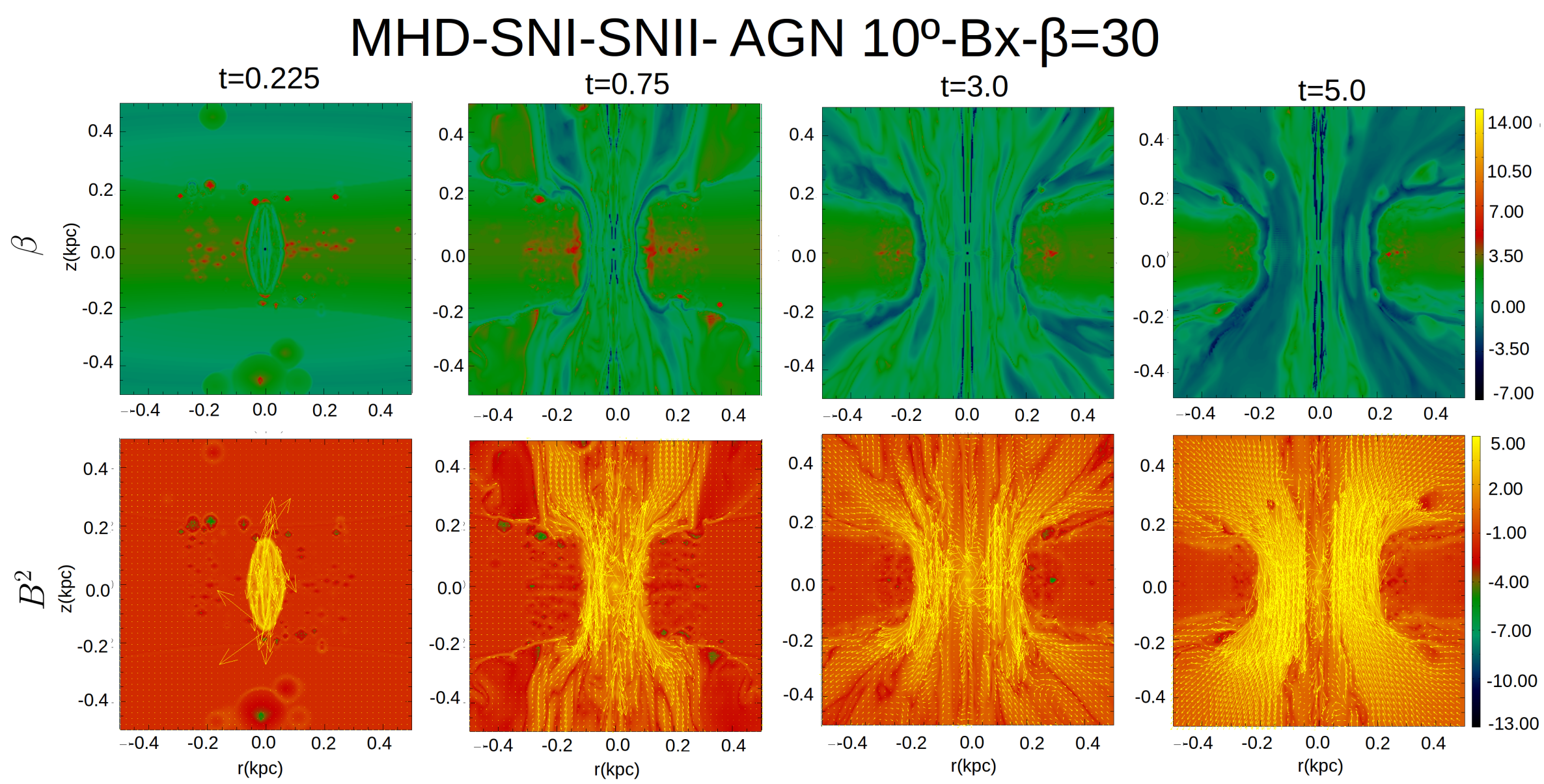

Figure 3.19: Edge-on logarithmic $2 \mathrm{D}$ cuts of the central slice of the model with SF-driven outflow, no-collimated AGN-wind (10 ${ }^{\circ}$ opening angle) and an inital horizontal magnetic field, named MHD-SN Ia-SN II-AGN $10^{\circ}-B_{x}-\beta_{30}$ for four diferent times of evolution: $t=0.225,0.75,3.0,5.0$ $\operatorname{Myr}$ (from left to right respectively). $\beta$ is adimensional (top). $B^{2}$ is in units of $2.40 \mu \mathrm{G}$ (bottom) 


\subsection{MHD Models}

In order to study the effect of the magnetic field on the system, in this section we introduce two different initial configurations for the magnetic field. One of them is parallel to the galactic disk pointing in the $\hat{x}$ direction. The second one is a magnetic field perpendicular to the plane of the disk in the $\hat{z}$ direction.

\subsubsection{MHD Models without AGN-wind (MHD-SN Ia-SN II- $B_{x^{-}}$ $\beta_{300}$ and MHD-SN Ia-SN II- $\left.B_{z}-\beta_{300}\right)$}

Figures 3.4-3.7 show the time evolution of 2D central cuts of density, velocity, temperature, thermal pressure, $\beta$ and the square of the magnetic field $B^{2}$ for the models above. The initial value of $\beta$ adopted is $\beta=300$. Figure 3.5 (at $t=5 \mathrm{Myr}$ ) of the model with an initial horizontal magnetic field, shows the presence of strong gradients in the magnetic field intensity (and magnetic pressure) near the disk midplane. These are associated with the magnetic field loops arising from the disk, as indicated by the yellow arrows that trace the field vectors. This is a clear evidence of the presence of the Parker-Rayleigh-Taylor instability (PRTI, see Parker (1979); Kadowaki et al. (2018)), which often occurs in stratified atmospheres which are sustained against gravity not only by the gas thermal pressure, but also by the magnetic pressure of a horizontal magnetic field, as in this model. The disk gas, initially in magnetostatic equilibrium, when perturbed by the formation of SNe bubbles, has its stability broken by the PRTI. We note that in the case of the model where the magnetic field is perpendicular to the disk plane and thus provides no extra support against gravity, this effect is absent.

As remarked, this local effect in the midplane is not large enough to affect the overall structure and evolution of the outflows, not at least for the initial value of $\beta$ considered here. Also, comparing the evolution of the magnetic fields and their intensities in both models of Figures 3.5 and 3.7, we note that they are very similar. The initial geometry in both is modified by the SF-wind gas expansion that drags the magnetic field lines to the vertical directions mainly at the highest altitudes. Besides, there is no obvious preservation of cold dense structures due to the shielding effects of the magnetic fields, not at least for these values of $\beta$. Smaller values (i.e., larger magnetic field strengths) may help to preserve more the structures in the winds (see more below). 


\subsubsection{MHD Model including a SF wind, a collimated AGN wind and a horizontal magnetic field (MHD-SN Ia-SN II-AGN $\left.\mathbf{0}^{\circ}-B_{x}-\beta_{300}\right)$}

Figures 3.8 and 3.9 show the evolution of this model which has an initial horizontal magnetic field with $\beta=300$. Compared with its hydrodynamical counterpart (model HDSN Ia-SN II-AGN $0^{\circ}$, Figure 3.2), we see that the AGN-wind propagation is delayed as shown at $t=0.225 \mathrm{Myr}$. The AGN wind has to break through an environment supported by an additional vertical pressure gradient force (besides the thermal pressure gradient $-\nabla p)$, i.e., the one due to the magnetic field gradient $-\nabla\left(B^{2} / 8 \pi\right)$. In other words, the outward bow shock that develops at the collimated wind head has to use part of its momentum to push the magnetic field lines and this delays its propagation with respect to the HD model. When the AGN-wind breaks through the disk, the bow-shock reaches vertical velocities between $2-3 \times 10^{3} \mathrm{~km} \mathrm{~s}^{-1}$.

At $t=0.75 \mathrm{Myr}$ the channel opened by the AGN-wind reaches a maximum diameter of $\sim 100$ pc near the basis, and removes about $99 \%$ of the initial mass from the core. This may temporarily quench accretion onto the SMBH and can represent a short episode in the evolution of the accretion because as soon as the SNe explosions heat the gas, this increases the AGN wind confinement (see Figure 3.8 at $t=3 \mathrm{Myr}$ ) and resumes the gas inflow to the central core and new fuel is available for accretion increasing the mass-loading between $t \sim 1.4 \mathrm{Myr}$ and $t \sim 3.5 \mathrm{Myr}$ (see Figure 3.26 black curve bottom panel).

When compared with its HD counterpart of Figure 3.2 at $t=0.75 \mathrm{Myr}$, we see that the width of the channel is broader for the magnetized model. This is because of extra magnetic forces in the AGN beam. As the initially horizontal magnetic field lines are dragged by the rotating plasma that is swept by the beam in the vertical direction, this quickly develop a helical structure with a well developed poloidal (vertical) component, as we see in Figure 3.9.

At this stage, the AGN-wind crosses the halo region and opens into a fountain wind. As in the HD counterpart model of Figure 3.2, this AGN wind is also stratified, being denser, colder and faster in the center of the beam with velocity, density and temperature ranging from $\sim 2 \times 10^{4} \mathrm{~km} \mathrm{~s}^{-1}$ to $\sim 150 \mathrm{~km} \mathrm{~s}^{-1}$ (from the center to the outer radius of the beam), $\sim 10^{-3}$ to $\sim 10^{-2} \mathrm{~cm}^{-3}$ and $10^{9}-10^{4} \mathrm{~K}$, respectively.

Later on, at $t=3 \mathrm{Myr}$, we see the formation of clumpy estructures in the interacting regions between the AGN and the SF winds located at hights of $z \sim 0.3-0.4 \mathrm{kpc}$ above and below the galactic disk, with densities of $10^{-1}-10 \mathrm{~cm}^{-3}$, vertical velocities of $v \sim$ $150 \mathrm{~km} \mathrm{~s}^{-1}$ and temperatures of $T \sim 10^{4} \mathrm{~K}$.

These structures are not as high and fast as in the HD model (Figure 3.2) due to local effects of the magnetic fields. Figure 3.20 depicts a detail of some of these structures 


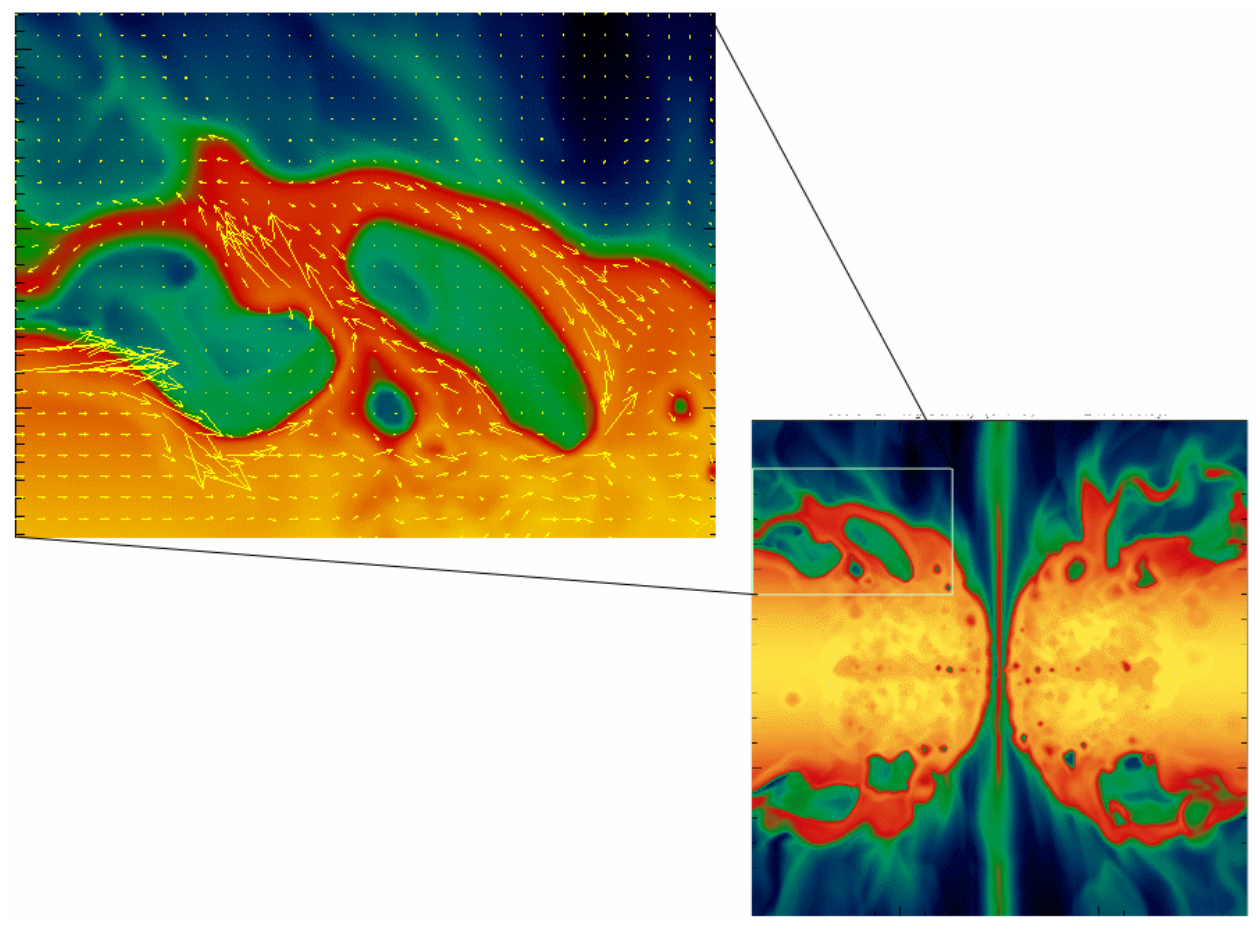

Figure 3.20: Detail of clumps at $z \sim 300 \mathrm{pc}$ in the 2D density map of the model MHD-SN Ia-SN II-AGN $0^{\circ}-B_{x}-\beta_{300}$ of Figures 3.8 and 3.9. The yellow arrows stand for the projected magnetic field vectors in the plane. Here, the clumps (red) are more preserved than in the HD model couterpart, because of the magnetic shielding.

that are outflowing from the galactic disk at this stage. We remark that the formation of clumps and fragments in the regions around the SN blobs immersed in the ISM and in the outflows are due to compression followed by non-uniform radiative cooling of gas behind the shocks. In the presence of magnetic fields, part of the energy of the shocks goes to the magnetic field, therefore decreasing the strength of the shocks and preserving more the clump structures from disruption.

Also, the temperature in the dense disk of the magnetized model is smaller than in the HD model over almost all the evolution. This again is due to the presence of the magnetic field that absorbs part of the kinetic energy of the shocks from the SNe, unlike the case of the HD model where this kinetic energy does not have to be shared and goes mostly to increase the internal energy (and temperature) of the gas. The presence of the magnetic fields in the AGN and SF winds combined, clearly affects their overall evolution. We find that the propagation velocity of the SF-driven wind and their structures are slightly smaller than in the HD counterpart due to the additional magnetic pressure support. The AGN wind that blows out into a fountain at the highest altitudes refrains the expansion and acceleration of the SF-driven outflow retaining more gas at lower altitudes than in the HD model (see Figures 3.2 and 3.8 at $t=5 \mathrm{Myr}$ ). The AGN-driven outflow, on the other hand, can reach larger vertical velocities than the HD counterpart (see more below). 


\subsubsection{MHD Model including a SF wind, a collimated AGN wind, and a vertical magnetic field (MHD-SN Ia-SN II-AGN $0^{\circ}-$ $\left.B_{z}-\beta_{300}\right)$}

Figures 3.10 and 3.11 depict snapshots of the model MHD-SN Ia-SN II-AGN $0^{\circ}-B_{z^{-}}$ $\beta_{300}$ which has the same initial conditions as in model MHD-SN Ia-SN II-AGN $0^{\circ}-B_{x}-\beta_{300}$ (of Figures 3.8 and 3.9), except for the initial magnetic field configuration that is now perpendicular to the disk plane (see Figure 2.3 left).

Despite the differences in the initial geometry, the comparison of the overall evolution of these two models shows that they are very similar. Differences arise mostly in local effects $^{1}$. As before, the lack of significant differences are due to the large initial value of $\beta$, which characterizes a dominance of the thermal effects over the magnetic fields in the dynamics of the gas. The latter easily drags the magnetic field lines in the gas outflows, in compressed and expanding regions and the resulting magnetic configuration quickly becomes similar in both models.

\subsubsection{MHD Model including a SF wind, an AGN wind with $10^{\circ}$ opening angle, and a horizontal magnetic field (MHD-SN Ia-SN II-AGN $\left.10^{\circ}-B_{x}-\beta_{300}\right)$}

This model is depicted in Figures 3.12 and 3.13. In the early phase evolution $(t \sim 0.25$ Myr), while the AGN-wind is propagating through the thick disk, it takes a little longer time than the HD counterpart (Figure 3.3), as expected, due to the presence of the extra magnetic support in the ambient gas. The velocity of the bow shock propagation is $v \sim 2000 \mathrm{~km} \mathrm{~s}^{-1}$. Figure 3.21 shows in detail this early phase evolution for this model when the AGN wind has just crossed the disk to the halo. We can clearly see the AGN wind bow shock, the internal collimation shocks along its beam (as described previously), and the type II supernova bubbles exploding into the disk and type Ia in the halo.

The AGN-driven shock that propagates along the dense disk is not as effective in heating the environment as in the HD counterpart model. Different from the HD-model where the shock propagates and deposited almost all its mechanical energy in the ISM, in this case, part of the shock energy goes into the magnetic field.

We also note that over all the expansion in time through the environment, the AGN outflow in this model is less collimated than in the HD model (Figure 3.3). This occurs because of the magnetic tension forces of the helical magnetic field that develops around the beam that opposes further confinement by the total external pressure gradient forces (see Figure 3.23). Figure 3.13 shows the corresponding values of the increased magnetic

\footnotetext{
${ }^{1}$ We note, for instance, that the model MHD-SN Ia-SN II-AGN $0^{\circ}-B_{z}-\beta_{300}$ does not show any evidence of the the growth of the PRI since the field is predominantly parallel to the acceleration of gravity.
} 

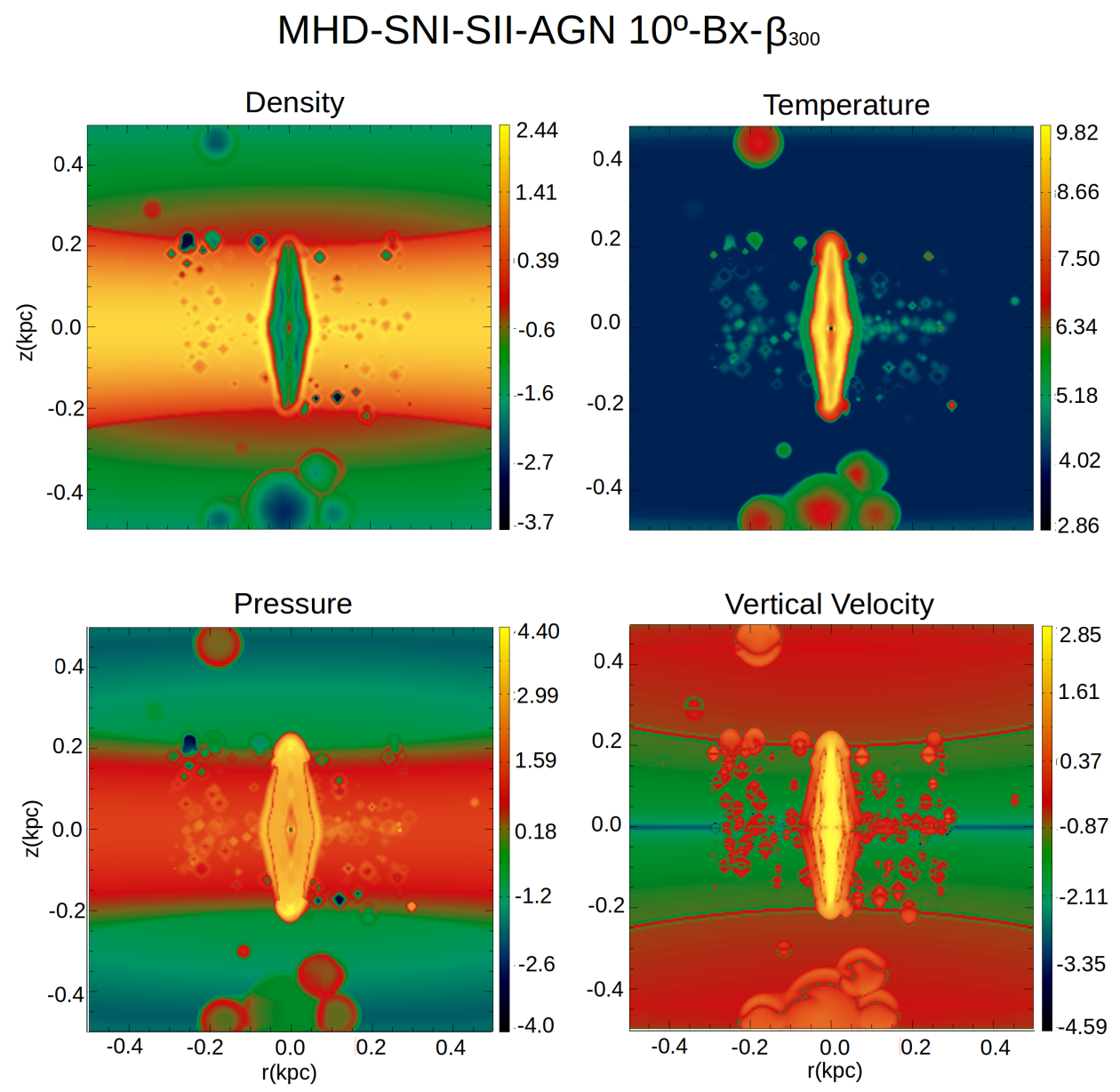

Figure 3.21: Detail of the early evolution of the model MHD-SN Ia-SN II-AGN $10^{\circ}-B_{x}-\beta_{300}$, at $\mathrm{t}=0.250 \mathrm{Myr}$ when the jet is just crossing the disk to the halo.

field strength and $\beta$ that attains values around 1 in this region. This smaller confinement of the AGN-wind, in turn, results in an inefficient transport of ambient gas by of the AGN wind. In fact, the rate of gas carried away is almost ten times smaller $\left(5 \mathrm{M}_{\odot} \mathrm{yr}^{-1}\right)$ than in the counterpart HD model $\left(50 \mathrm{M}_{\odot} \mathrm{yr}^{-1}\right)$ (see also the red and green curves in Figures 3.27 and 3.28).

The time-scale taken by the AGN-wind to leave the $1 \mathrm{kpc}$ region is $t \sim 0.29 \mathrm{Myr}$, opening a channel of $\sim 120$ pc of diameter around the center at the early stages of evolution $(t \sim 0.75 \mathrm{Myr})$ and reaching a maximum diameter of $\sim 400 \mathrm{pc}$ at later stages $(t \sim 5.0 \mathrm{Myr}$ ), which is larger than the HD model (Figure 3.3). In the first $0.5 \mathrm{Myr}$, the jet removes $\sim 99 \%$ of the initial mass in the central core which quenches accretion onto the SMBH (see also Figure 3.26 green curve). However, contrary to the HD counterpart and the other models, this is not a temporary effect in this case. In this model, the confining pressure and gas feeding provided by the SN II explosions in the disk are not enough to fuel again the SMBH, so that there is a permanent quenching of gas inflow (accretion) (see Figure 3.12 first and fourth rows for density and pressure at $t=5.0 \mathrm{Myr}$ ).

As the AGN wind impinges the halo and reaches the $1 \mathrm{kpc}$ scales at $t \sim 0.75 \mathrm{Myr}$ 
(Figure 3.12) the generated wind has densities between $10^{-3}-1 \mathrm{~cm}^{-3}$, velocities between $\sim 300 \mathrm{~km} \mathrm{~s}^{-1}$ and $\sim 30000 \mathrm{~km} \mathrm{~s}^{-1}$ and temperatures from $\sim 10^{6}-10^{9} \mathrm{~K}$. These highest velocities and temperatures resemble the properties of the observed UFOs (e.g. Tombesi et al. (2015); Kraemer et al. (2018); see more below). While the injected AGN-wind is accelerated to velocities up to $\sim 10.000 \mathrm{~km} \mathrm{~s}^{-1}$, the massive SNe-driven wind is accelerated to velocities $\sim 300-1000 \mathrm{~km} \mathrm{~s}^{-1}$, and they both are accelerated by the pressure gradients and magnetic tension forces.

We note that the AGN wind changes as it evolves. At $t=0.75 \mathrm{Myr}$, the AGN wind is more collimated and has an opening angle of $\sim 10^{\circ}$ above $z \geq 200 \mathrm{pc}$. At $t=5.0 \mathrm{Myr}$ the AGN-wind has an opening angle of $45^{\circ}$.

After $t \sim 3$ Myr we see in Figure 3.12 that the outflow is entirely governed by the AGN-wind. The SF activity is almost quenched around the nuclear region of the galaxy and the expanding more massive SF-wind is pushed back towards the galaxy by the AGN-wind, at the highest altitudes, thus leading to partial fallback.

We also note in Figure 3.12, that the system at this stage exhibits an asymmetry with respect to the polar axis. This is triggered by the non-axisymmetric random explosions of SN II that we see earlier on the disk (at $t=0.225 \mathrm{Myr}$ ). This asymmetry causes an unbalancing on the external pressures that confine the AGN outflow on both sides leading to the slight asymmetry, later on, described above (see Figure 3.22).

\subsubsection{MHD Model including an SF wind, an AGN wind with $10^{\circ}$ opening angle, and a vertical magnetic field (MHD- SN Ia-SN II-AGN $10^{\circ}-B_{z}-\beta=300$ )}

This model is depicted in Figures 3.14 and 3.15 and can be directly compared with its HD counterpart (Figure 3.3) and the one with same initial conditions except for the presence of an initial parallel magnetic field (Figure 3.12). As in the case of the models with collimated AGN outflows, the models with initial $B_{z}$ and $B_{x}$ are morphologically very similar. The initial vertical field corresponding to a plasma $\beta=300$ that is thermal pressure-dominated, as in the model with $B_{x}$, is easily dragged by the motion of the rotating gas and soon develops a helical geometry, similar to that of a model with $B_{x}$ (see the projected magnetic field poloidal vectors in Figure 3.15 and helical magnetic field lines in 3.23).

As in the model with $B_{x}$, we also see that the AGN jet takes longer to reach the halo than in the HD counterpart, again due to the magnetic tension and pressure forces that decelerate the flow a little (see Figures 3.12 and 3.14 at $t=0.225 \mathrm{Myr}$ ). The bow shock velocity at this stage is $\sim 1000 \mathrm{~km} \mathrm{~s}^{-1}$.

At $t=0.75 \mathrm{Myr}$ the passage of AGN wind by the halo, generates a wind which reaches velocities ranging between $\sim 5000 \mathrm{~km} \mathrm{~s}^{-1}$ and $\sim 20000 \mathrm{~km} \mathrm{~s}^{-1}$, and temperatures from 


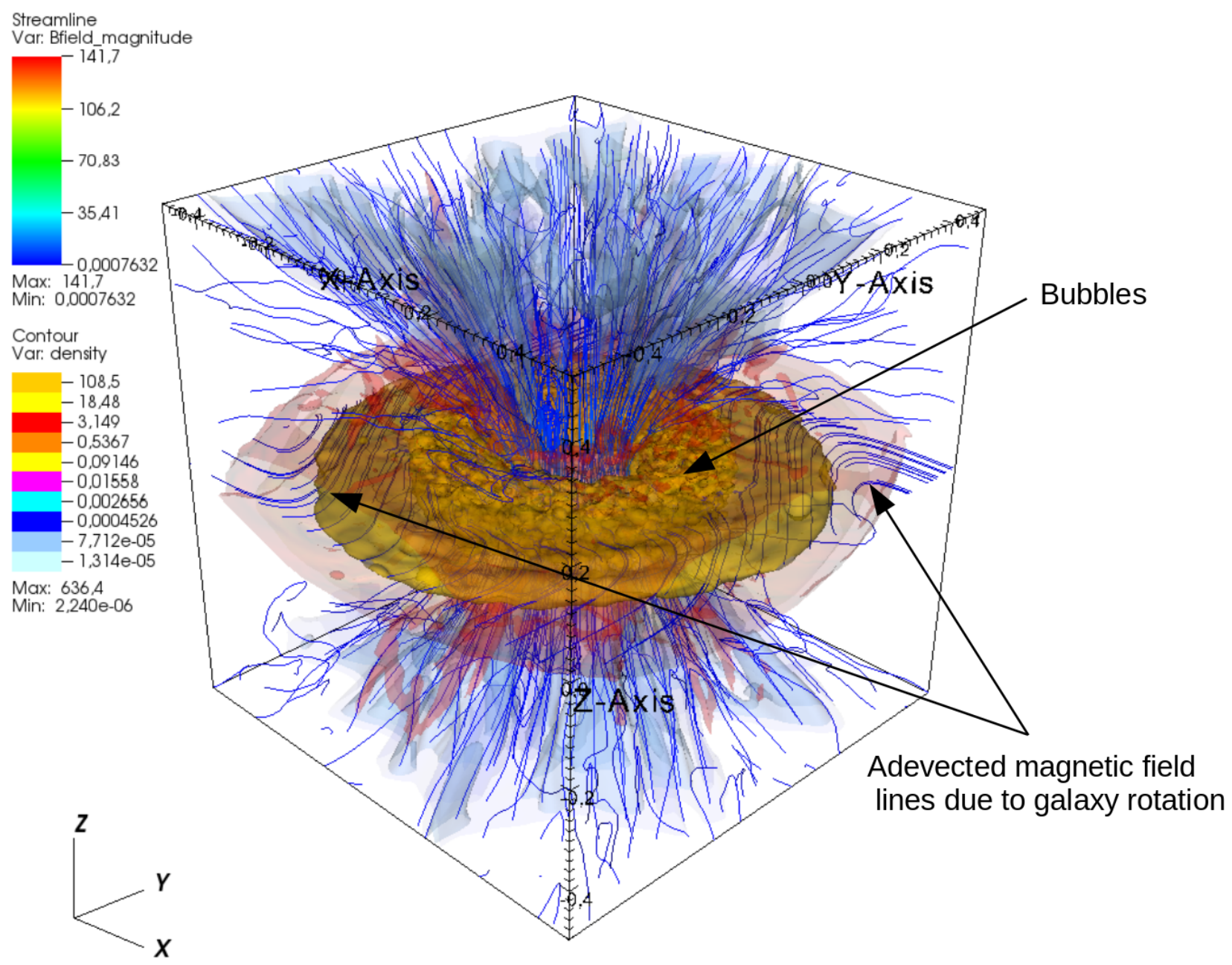

Figure 3.22: $3 \mathrm{D}$ volume density contour plot for the model MHD-SN Ia-SN II-AGN $10^{\circ}-B_{x^{-}}$ $\beta_{300}$ at $t=5 \mathrm{Myr}$ showing SN bubbles in the disk and the SF-driven wind that is much more massive than the AGN-wind not seen here because its much smaller density. The magnetic field lines are represented as blue lines. Top bar color gives the density in $\mathrm{cm}^{-3}$ and bottom bar color gives the magnitude of magnetic field $|B|$ in units of $0.76 \mu G$

$\sim 10^{6}-10^{9} \mathrm{~K}$, thus also similar to the model with the initial horizontal magnetic field.

\subsubsection{Comparison of MHD models with different initial values of $\beta$}

In all the MHD models studied so far, we have adopted an initial $\beta$ value that corresponds to a magnetic field that can be considered a typical average value in spiral and Seyfert galaxies. In this section, for completeness, in order to explore further the potential effects of magnetic fields, we consider a model with initial $\beta$ value 10 times smaller $(\beta=30$ corresponding to initial $B_{x}=2.4 \mu \mathrm{G}$, see table 2.2 ), in order to compare directly with the model with $\mathrm{AGN}$ wind with $10^{\circ}$ opening angle and initial horizontal field (see Figures 3.18 and 3.19). When compared with the $\beta=300$ model (Figures 3.12 and 3.13), we see that there are no major differences between them in the macroscopic morphological 


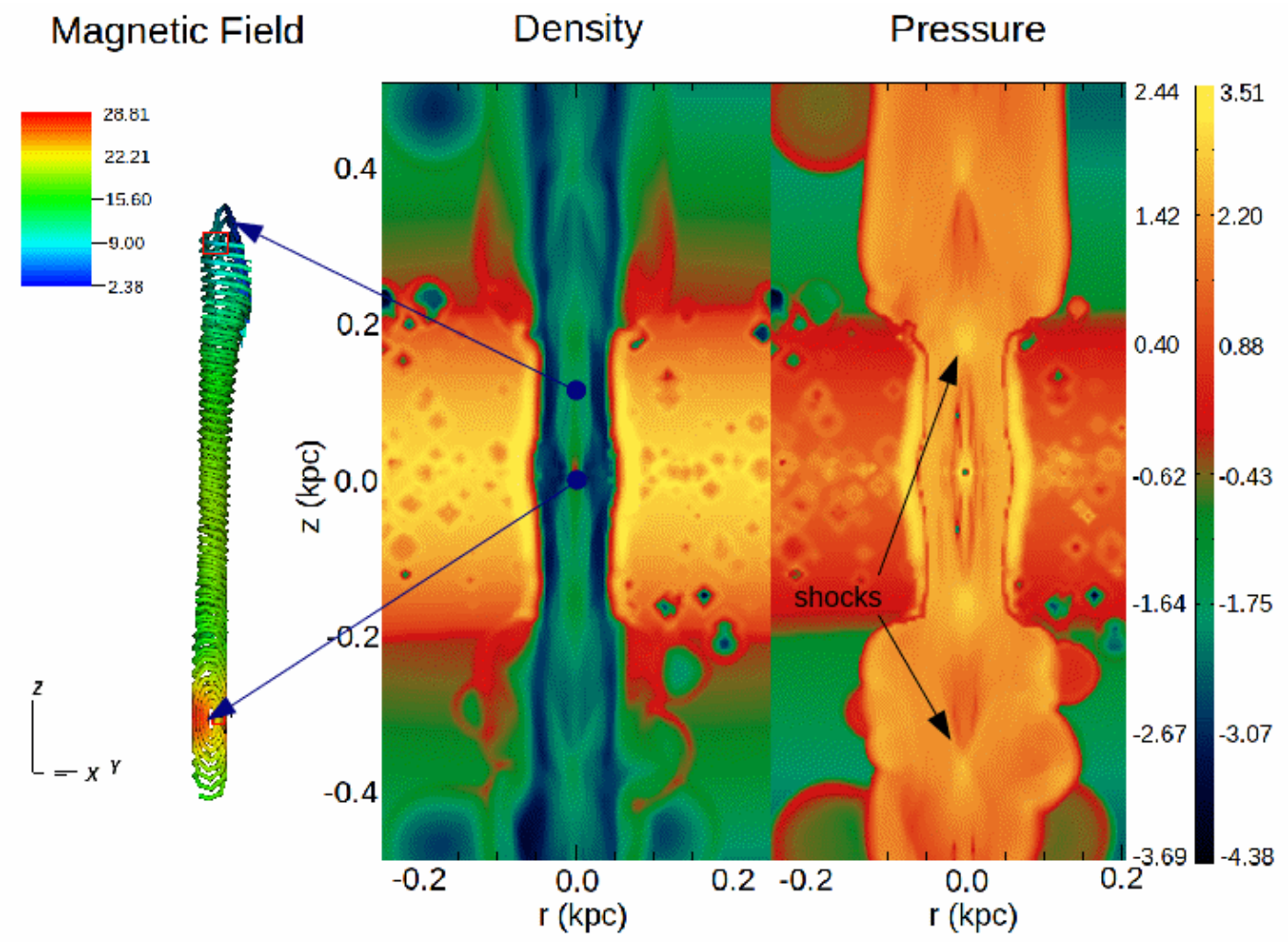

Figure 3.23: Left: $3 \mathrm{D}$ helical magnetic field lines warped around the jet. $2 \mathrm{D}$ logarithmic slices for density $\left(\mathrm{cm}^{-3}\right)$ and thermal pressure (middle and right respectively). Four recollimation shocks are formed over $\sim 0.2 \mathrm{pc}$ and $\sim 0.4 \mathrm{pc}$ from the central region at the northern and southern jets. As the jet expands laterally, its thermal pressure decreases with distance along the direction of propagation. When the jet pressure reaches the ambient pressure the jet begins to re-collimate. The jet periodically expands and re-collimates, producing a series of biconical or normal shocks along the jet axis (for further detail, see, .e.g., Nawaz et al. (2014)). Four of these shocks appear as knots in the pressure plot at right. Logarithmic scale for density is at left and for pressure is at right. Density is in $\mathrm{cm}^{3}$, pressure in reference units $7 \times 10^{12} \mathrm{erg} \mathrm{cm}^{-3}$, and magnetic field in units of reference, $0.76 \mu \mathrm{G}$.

features or the quantities, as time evolves. We have also performed simulations of MHD models with collimated AGN winds, considering different initial values of $\beta=30$ (Figures 3.16 and 3.17), and $\beta=3$ (no shown here), and the results found are also similar, i.e, we have not identified substantial differences with regard to the $\beta=300$ model. This will reflect in the results reported in the next section where we investigate the AGN and SF feedback on the galaxy evolution by quantifying average quantities in the models.

\subsection{Discussion and analysis of AGN and SF Feedback}

In this section, we quantify total and average values of important quantities that can be directly compared with observations of molecular outflows and UFOs. 


\subsubsection{Average number density}

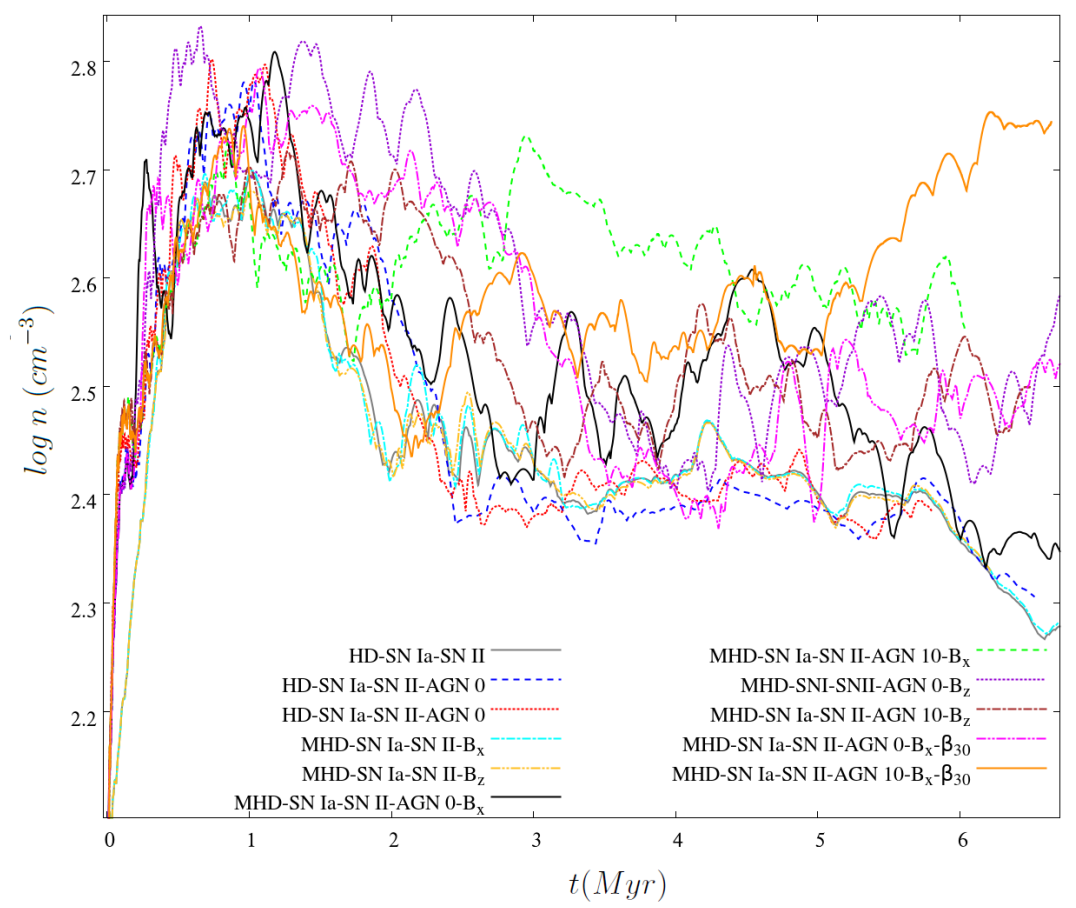

Figure 3.24: Volume averaged number density in the systems as a function of time. Density is in $\mathrm{cm}^{-3}$ and time in Myr. The colors of the curves stand for the models according to the legend.

Figure 3.24 depicts the average number density evolution for the models. This average was calculated in each time step by summing the densities in each cell of the domain and then dividing by the total number of cells. We see that the highest average densities in all models are achieved in the first million year. The highest density features in the system are formed by compressed gas of the ISM by SN shocks and by the bow shock generated by the AGN wind when still propagating inside the disk. After the first million year, most of these compressed (and also radiatively cooled) features are evaporated and the average density decreases slowly until $t \sim 2.5 \mathrm{Myr}$ and then remains almost constant for most of the models with small variations.

\subsubsection{Volume avergae of $\beta$}

Figure 3.25 shows the average value of the $\beta$ ratio in the MHD models as a function of time. As this ratio evaluates the relative importance of the thermal over the magnetic pressure in the system, we clearly see that only in the models with AGN wind with $10^{\circ}$ opening angle is that this ratio attains average values which are smaller than one, therefore characterizing the dynamical importance of the magnetic fields in these systems, as we describe in the other sections. 


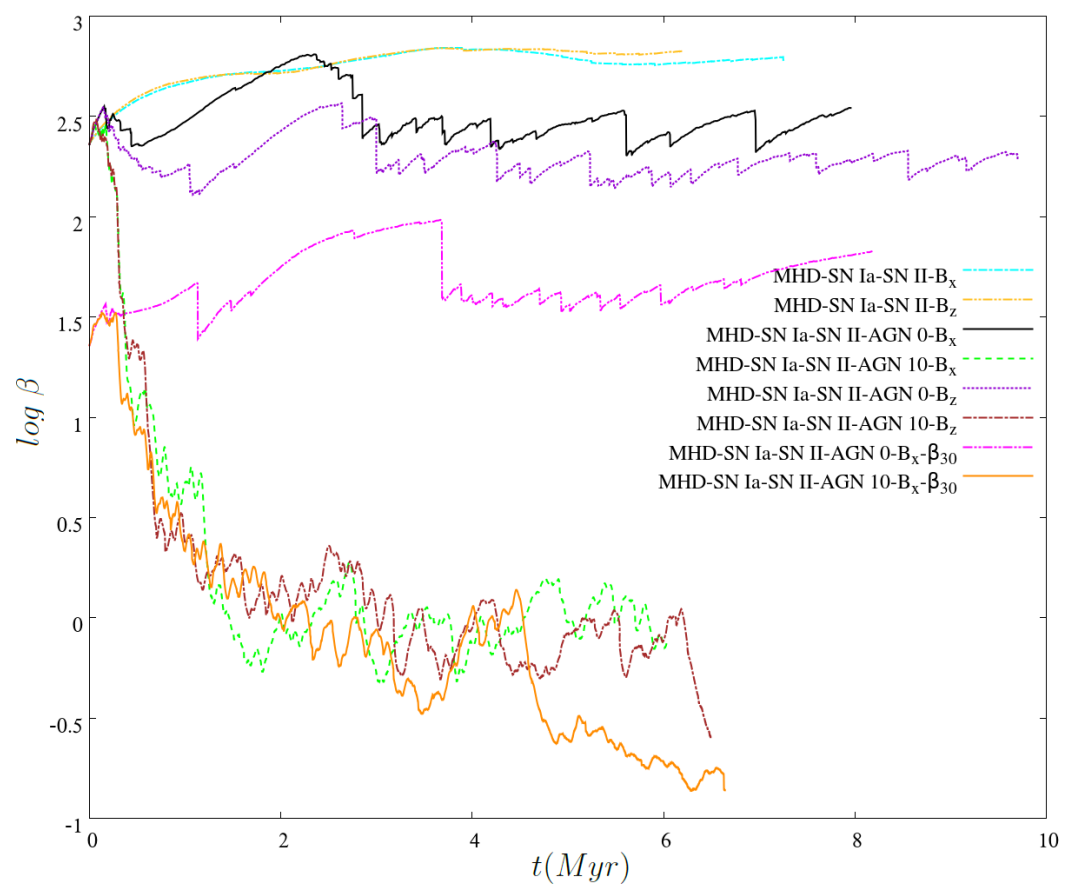

Figure 3.25: Volume averaged value of $\beta$ in the models as a function of time. Time is in Myr. The colors of the curves stand for the models according to the legend.

\subsubsection{Mass Transport}

Figures 3.26 and 3.27, respectively, depict the temporal evolution of the total mass in the system considering two diferent regions: the first one is the central core region located at $r \leq 40 \mathrm{pc}$. The second one, the region located at $|z| \leq 200 \mathrm{pc}$, which corresponds to the central disk. These figures help us to understand how the gas mass is transported and redistributed through the system as it evolves ${ }^{2}$

The total mass evolution in the central core (Figure 3.26) for the models with no AGNwind (jet), i.e., HD-SN Ia-SN II and its MHD counterparts MHD-SN Ia-SN II- $B_{x}-\beta_{300}$ and MHD-SN Ia-SN II- $B_{z^{-}} \beta_{300}$ shows that there is not substantial differences introduced by the presence of fiducial magnetic fields. The removal of the mass from this region is very small in these models and mainly due to the SNe-driven wind. When a collimated AGN-wind (jet) is introduced in the system (HD-SN Ia-SN II-AGN $0^{\circ}$ model, dark blue curve), this removes $\% 95$ of the core mass in the first $\sim 0.25 \mathrm{Myr}$. After this period there is an increase in the mass in the next $0.75 \mathrm{Myr}$, due to new supply of gas inflow to the core, reaching $\% 90$ of its initial mass. This mass-loading the central core is due to the interstellar gas injection that is driven when the SN II explosions start to occur around this region. After this time, this mass replenishment in the core remains nearly constant. When magnetic fields are present in such models with collimated AGN winds

\footnotetext{
${ }^{2}$ The mass in each region is calculated by summing the gas mass in each cell of the domain, at each time step. So, when some amount of gas leaves some of these regions, it is counted as mass-loss and used to compute the mass-loss rate, like in Figure 3.28.
} 


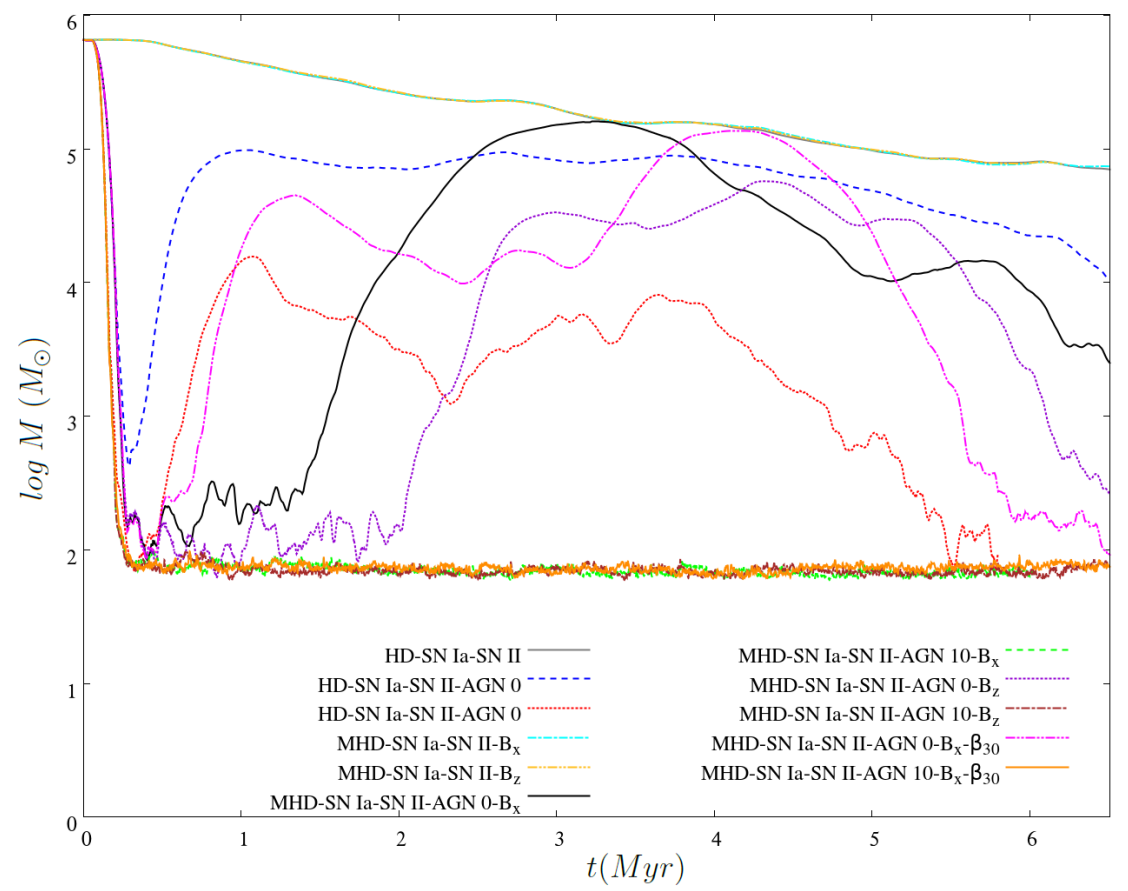

Figure 3.26: Total mass within the core region of the galaxy $(r \leq 40 \mathrm{pc})$ as a function of time for all models investigated in this work with inital $\beta \equiv \infty, 300$ and 30 (see Table 2.2). The colors of the curves stand for the models according to the legend.

(MHD-SN Ia-SN II-AGN $0^{\circ}-B_{x}-\beta_{300}$ and MHD-SN Ia-SN II-AGN $0^{\circ}-B_{z}-\beta_{300}$ black and purple curves, respectively) the situation is similar except that more mass is lost and the resupply of mass to the core occurs later, at $t \sim 1.5 \mathrm{Myr}$ in the first case, and $t \sim 2$ Myr in the second. This delay can be understood if we go back to Figures 3.8 and 3.10 of these models, where we have seen that the presence of the internal magnetic pressure gradient and tension forces in the AGN jet refrains the gas inflow from the ISM. This also explains the smaller mass inflow to the core after these periods in these magnetized models, in Figure 3.26. On the other hand, if we introduce a non-collimated AGN-wind into the magnetized models MHD-SN Ia-SN II-AGN $10^{\circ}-B_{x}$ and MHD-SN Ia-SN II-AGN $10^{\circ}-B_{z}$ (green and brown curves, respectively,) this core mass depletion is more extreme. After the initial removal of gas by the AGN wind, there is no further replenishment. This again is due to the internal magnetic forces here combined with the larger opening angle of the AGN wind, that effectively quench the interstellar gas inflow to the SMBH and even the SF near the nuclear region (see sections 3.2.4 and 3.2.5).

The mass evolution in the central disk is depicted in Figure 3.27. The light-blue, yellow and grey curves are overlapped and correspond to HD-SN Ia-SN II, MHD-SN Ia-SN II$B_{x^{-}} \beta_{300}$ and MHD-SN Ia-SN II- $B_{z^{-}} \beta_{300}$, respectively. We see that the mass removal by the SNe-driven outflow is very substantial in these models and is maximum after around 3 Myr when the wind driven by star formation has fully developed. Figure 3.28 depicts the corresponding mass-loss rates $d M / d t$ in the disk for the models, and the maximum value for these models with no AGN wind are $\sim 50 \mathrm{M}_{\odot} \mathrm{yr}^{-1}$. We further notice that as 


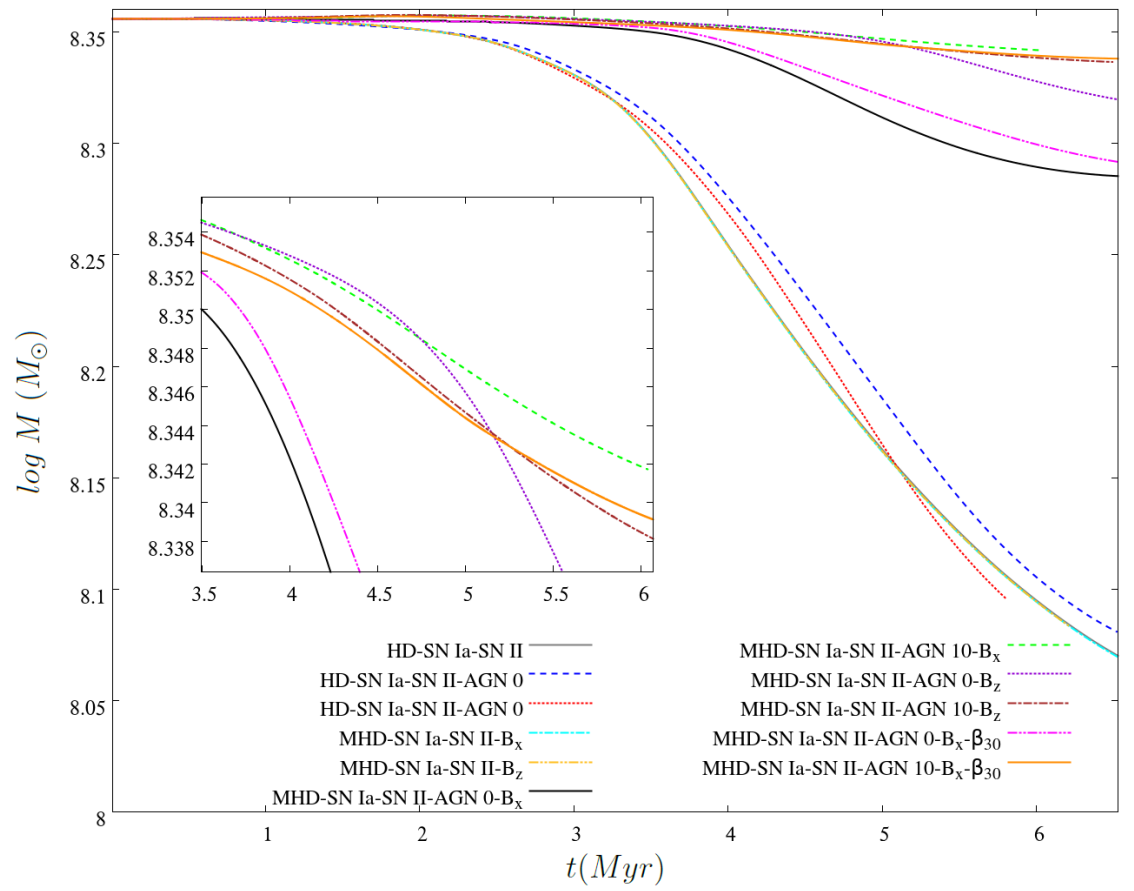

Figure 3.27: Total mass within the disk region of the galaxy $(|z| \leq 200 \mathrm{pc}$,$) as a function of$ time for all models investigated in this work with inital $\beta \equiv \infty, 300$ and 30 (see Table 2.2). The colors of the curves stand for the models according to the legend.

in the core, the presence of the magnetic field has no significant effect. If we compare the HD-SN Ia-SN II and HD-SN Ia-SN II-AGN $0^{\circ}$ models (grey[overlaped with golden and blue-light curves] and dark-blue curves) the presence of the collimated AGN-wind makes the transport of gas from the dense disk a little less effective. Examining the grey and dark-blue curves for the same models in Figure 3.28, we see that while there is a maximum mass lost rate of $\sim 45 \mathrm{M}_{\odot} \mathrm{yr}^{-1}$ for the model with collimated AGN-wind, there is a larger maximum loss rate of $\sim 50 \mathrm{M}_{\odot} \mathrm{yr}^{-1}$ when the AGN-wind is absent. This, as dicussed previously, is due to the development of the AGN outflow fountain-like structure that increases the thermal pressure over the expading SF-wind at the highest altitudes, causing the fallback of part of the SF-wind material to the disk (see pressure panels at $t=0.75$ and 3 Myr in Figures 3.1 and 3.2).

Now, the presence of magnetic fields in models with collimated AGN winds (MHDSSN Ia-SN II-AGN $0^{\circ}-B_{x}-\beta_{300}$ and MHD-SN Ia-SN II-AGN $0^{\circ}-B_{z}-\beta_{300}$ models) changes this situation considerably. The black and purple curves in Figures 3.27 and 3.28 show that, as in the case of the core and for the same reasons stressed there, these magnetized models with collimated AGN wind lead to a smaller mass loss from the disk in comparison to the HD model counterpart (dark-blue curve). Furthermore, we see that in the presence of an initial horizontal magnetic field $\left(B_{x}\right)$, the maximum loss rate is $\sim 20 \mathrm{M}_{\odot} \mathrm{yr}^{-1}$, i.e., almost twice larger than in the presence of a vertical magnetic field $B_{z}\left(\sim 10 \mathrm{M}_{\odot} \mathrm{yr}^{-1}\right)$, so that, the predominance of vertical magnetic field is less effective in removing the gas 


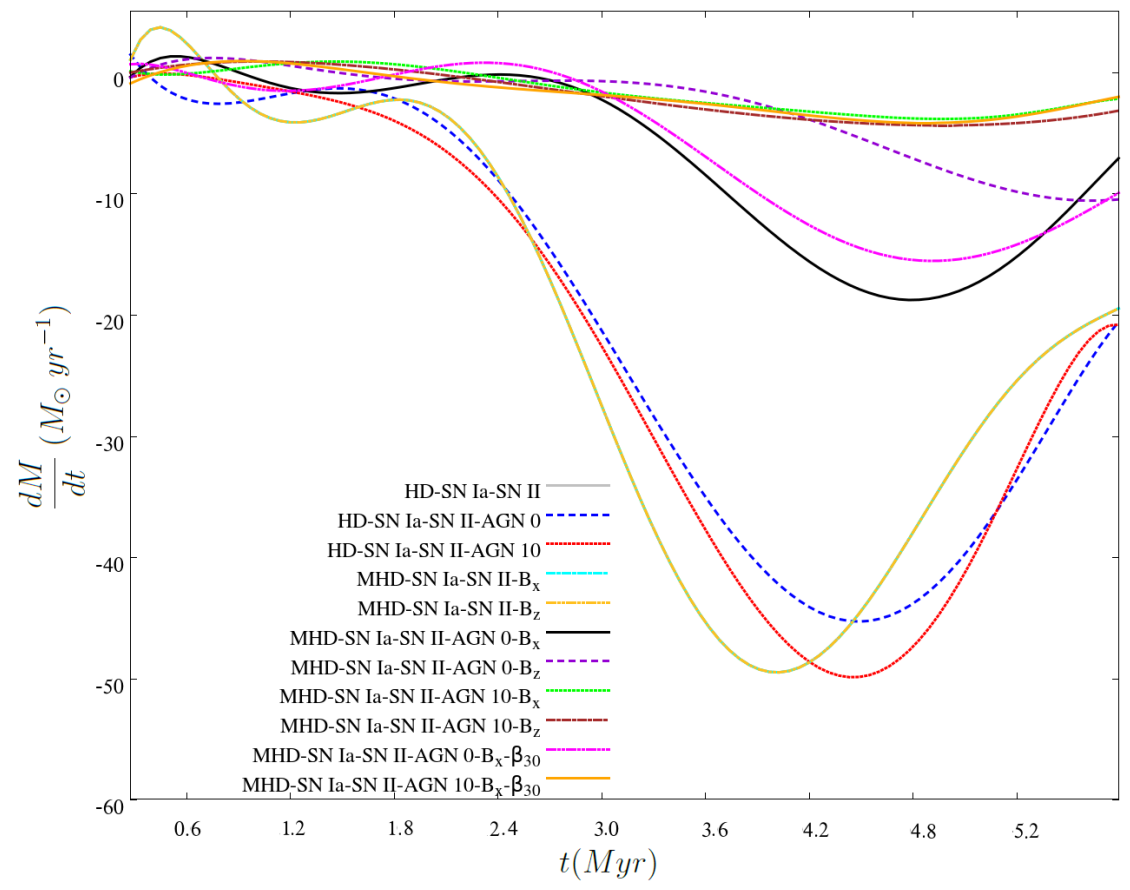

Figure 3.28: Time evolution of the gas total mass loss rate from the galaxy thick disk $(|z| \leq 200$ pc) for all the models. Time is in Myr, and mass-loss rate is in units of $\mathrm{M}_{\odot} \mathrm{yr}^{-1}$. The plot at the bottom left, shows a zoom on the curves for the models MHD-SN Ia-SN II-AGN $10^{\circ}-B_{x^{-}}$ $\beta=300$ (green), MHD-SN Ia-SN II-AGN $10^{\circ}-B_{z^{-}} \beta=300$ (brown) and MHD-SN Ia-SN II-AGN $10^{\circ}-B_{x^{-}} \beta=30$ (orange curve).

from the dense disk. This occurs because the predominance of horizontal magnetic field (and the associated magnetic pressure gradient support) in the thick disk in the first 3 Myr causes more resistence against gas removal by the AGN and SF winds than in the model with dominance of vertical fields (see also section 3.2.2).

The effects above are even more dramatic in the models with large AGN-wind opening angle. In the absence of magnetic fields, the model with AGN wind with $10^{\circ}$ opening angle (red curve), HD-SN Ia-SN II-AGN $10^{\circ}$, follows the same trend as detected for the HD model with collimated AGN wind (dark-blue curve), ie., it has little influence in the mass transport in the broad disk which is dominated by the SF-wind component. Comparing this model (red curve) with its MHD counterparts, the MHD-SN Ia-SN II-AGN $10^{\circ}-B_{x}$ and MHD-SN Ia-SN II-AGN $10^{\circ}-B_{z}$ models (brown and green curves in Figures 3.26 and 3.28), we see that that the mass loss rate much smaller in the MHD models, almost ten times smaller $\left(5 \mathrm{M}_{\odot} \mathrm{yr}^{-1}\right)$. As we have seen in section 3.2.4 and 3.2.5 and Figures 3.12-3.15, the large channel opened by the AGN winds in these models, helped by the extra internal forces due to the magnetic fields (which have small local values of $\beta \sim 10^{-1}-10^{-2}$ ), cause the eruption of the outflow at the highest altitudes into a large fountain (with an opening angle $\sim 45^{\circ}$ ). This fountain refrains the expanding massive plasma of the disk (which is being driven by the SF wind component) and makes part of this outflowing gas to fallback into the galaxy, therefore decreasing the mass loss with 
regard to the HD counterpart.

The main results of this section with regard to mass transport can be summarized as follows:

- The presence of a collimated AGN wind causes significant removal of mass from the core region in a few $100.000 \mathrm{yr}$, but this is replenished later on by gas inflow when the SNe explosions in the surrounds are fully developed. A large opening angle AGN wind, on the other hand, particularly when helped by the presence of magnetic fields that further resist to the external confining pressure gradients, is able to remove the nuclear gas entirely within a few $100.000 \mathrm{yr}$ and does not allow for later replenishement, therefore quenching fueling and mass accretion onto the SMBH. These results indicate that the dute cycle of these outflows is around a few $100.000 \mathrm{yr}$, which is also compatible with the typical dynamical time-scales inferred for the observed UFOs and molecular outflows, as described in Chapter 1 (see also, Gofford et al. (2013), Tombesi et al. (2015); Morganti et al. (2013), Tadhunter et al. (2014), Kraemer et al. (2018)).

- The mass loading of the outflows is dominated by the SF-wind component. Maximum loss-rates from the disk of the order of $50 \mathrm{M}_{\odot} \mathrm{yr}^{-1}$ are obtained which are compatible with the observed values in molecular outflows, as described in Chapter 1.

- The AGN wind alone has little influence in the mass loading of the outflows (as verified already by Melioli and de Gouveia Dal Pino (2015)), but combined with the presence of magnetic fields, it is able to refrain the mass transport from the disk to the highest altitudes because of the formation of a broad fountain that retains part of the gas in the galaxy (fallback), therefore decreasing the mass loading in the outflows to values that are smaller the larger the AGN wind opening angle (up to a factor 10).

\subsubsection{Average Velocity}

Figure 3.29 depicts the volume averaged velocities of the models investigated in this work. In the early years of evolution (Figure 3.29 (a) ), we see that the models are separated in three branches. Initially, the acceleration is very slow and similar in all models. In the models that have an AGN wind, once this reaches the halo, around $\sim 0.25$ Myr,the velocity grows exponentially as the wind breaks through the disk to the halo. This exponential growth is larger for larger AGN-wind opening angle, separating the models with $10^{\circ}$ and $0^{\circ}$ opening angle in the diagram in two branches (see the red, green and brown curves for the models with $10^{\circ}$ degree opening angle; and the dark-blue, black and 
violet for the models with $0^{\circ}$ opening angle AGN winds). After this exponential phase, the velocity keeps increasing, but more slowly until reaching a near steady-state after $\sim 1$ Myr for most of the models (see Figure $3.29 \mathrm{~b}$ ). The models with the outflows driven only by SF-winds (grey, yellow, and light-blue curves) are in a third branch and have a continous, slower increase in velocity in the early evolution until a maximum around 3.5 Myr (Figure 3.29 b), typical of SNe or SB-driven winds (see e.g., Melioli et al. (2013); Geraissate (2010), and references therein for a detailed study on SF-driven winds theory and evolution). We also note that the presence of the magnetic fields have no effect on the inital acceleration of the outflows, not at least for the value of $\beta$ here considered $(\beta=300)$.
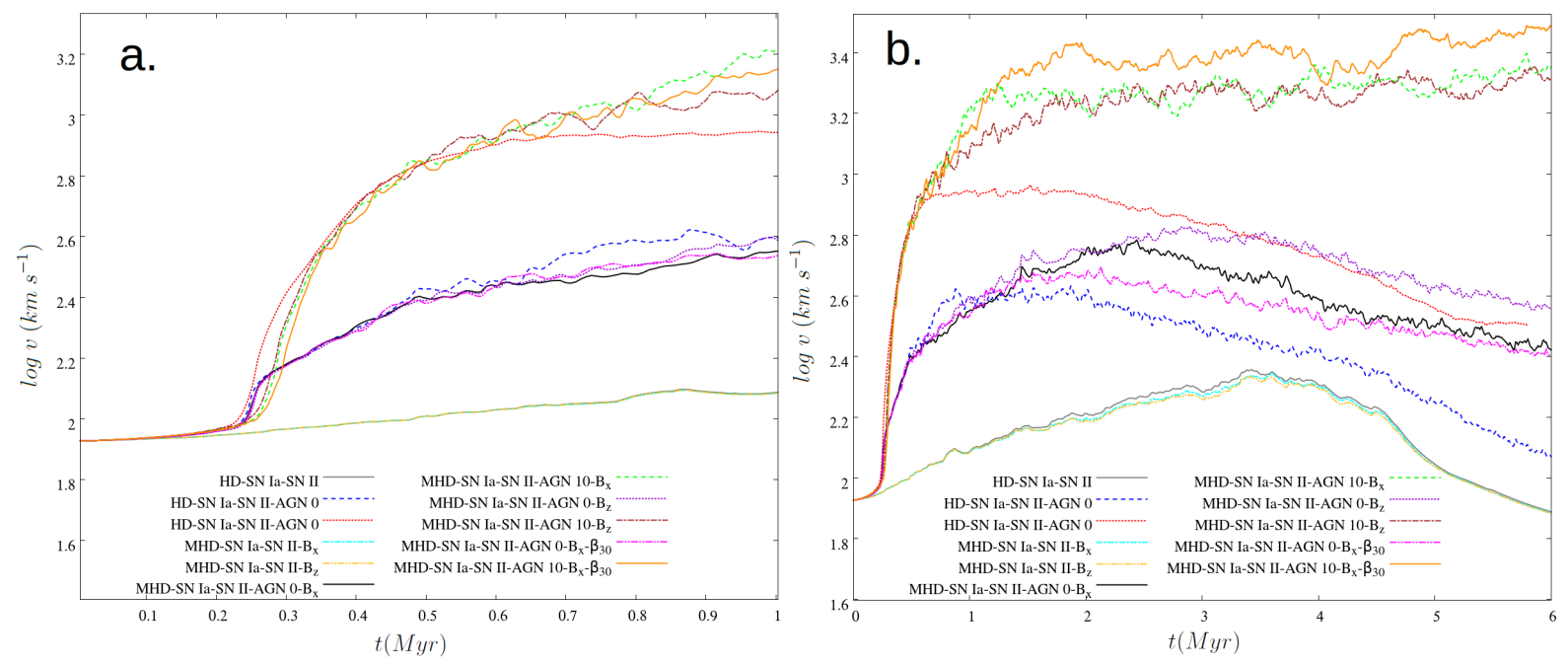

Figure 3.29: Volume average velocity for the models: a. evolution over the first $1.0 \mathrm{Myr}$, and b. evolution over 6 Myr. The velocity is calculated by summing the velocities of each cell of the domain and dividing by the total number of cells.

In the late evolution, in Figure 3.29 (b), we can still distinghish the three main branches, though the presence of magnetic fields causes some spreading within the original branches. For instance, we clearly see that both magnetized models with larger AGNwind opening angle attain the largest velocities and keep them nearly constant (green and brown curves), while their HD counterpart (red curve) suffers some decelaration after $1 \mathrm{Myr}$ and its velocity approaches that of the branch of the collimated AGN-wind models after $\sim 3$ Myr. The branch of the collimated AGN-wind models (dark blue, violet and black curves) also suffers some spreading and decelaration after $\sim 3$ Myr, with the HD counterpart being more decelerated than the magnetized models, approaching the branch of the models with SF-wind only. The inspection of the 2D distributions for these models in the figures of section 3.2 shows that around this time the upper region that was previously occupied by hot fast halo gas is now mixed with colder, denser and slower gas that was carried by the SF-wind from the disk, thus making the average velocity to decrease.

Figures 3.30 and 3.31 show 2D histrograms of velocity versus density (upper panels), 

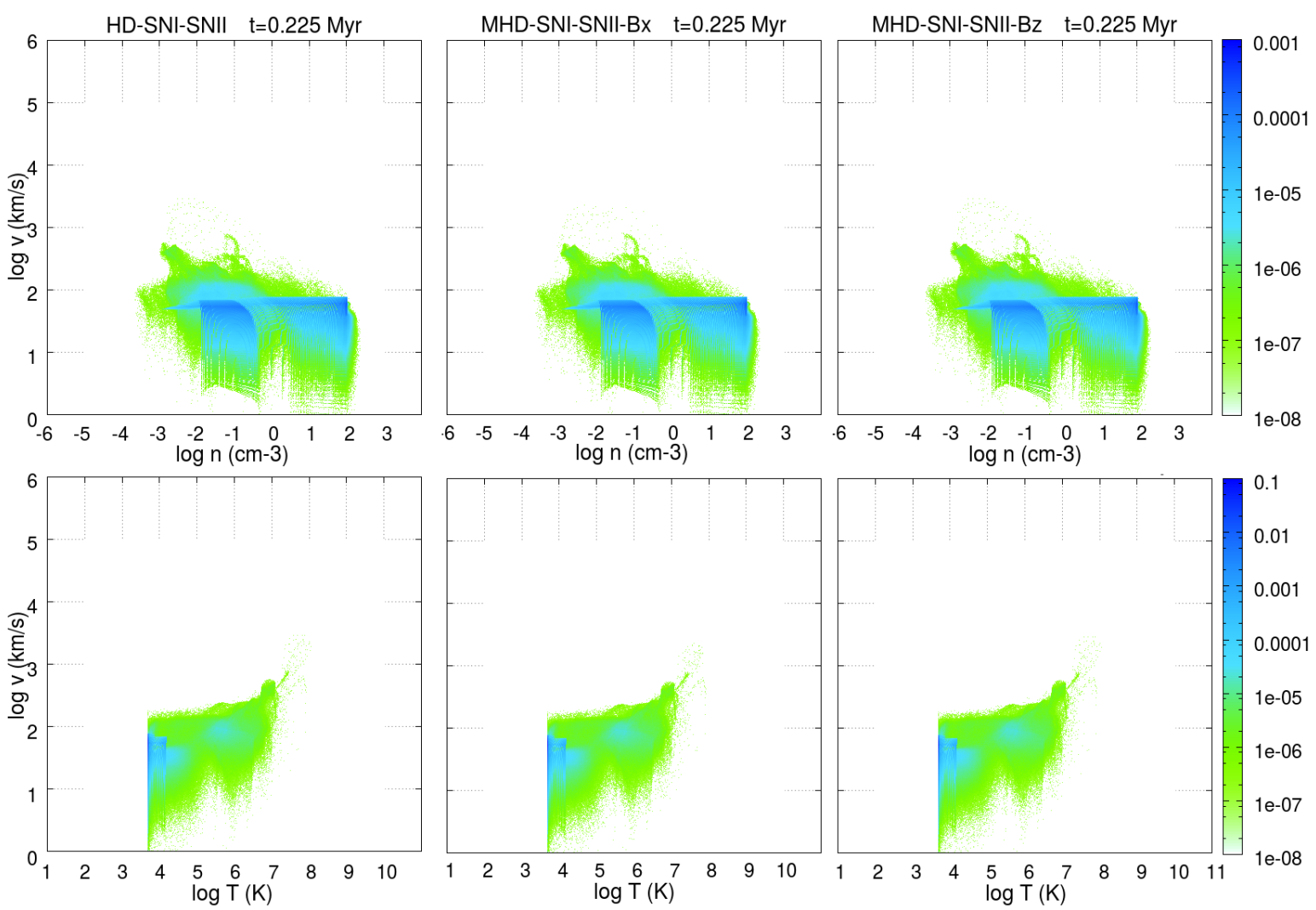

Figure 3.30: Two dimensional histograms of the vertical velocity versus density (V-n) (top), and velocity vs. temperature (V-T) (bottom) calculated considering every cell within the whole simulated system at $t=0.225 \mathrm{Myr}$, for the three models with SF-wind only, from left to right: the HD model, the model with initial horizontal magnetic field, and the model with initial vertical field, both with $\beta=300$. Velocity is in $\mathrm{km} \mathrm{s}^{-1}$, density in $\mathrm{cm}^{-3}$ and temperature in $\mathrm{K}$, all in logarithmic scale. The color bar indicates the cell number normalized to their total number. The names of the models are indicated at the top of each panel.

and temperature distributions (bottom panels) for the entire domain of the models with $\beta=300$ and SF-wind only, at $t=0.225$ and $5 \mathrm{Myr}$, respectively, while Figures 3.32 and 3.33, show the same for the models with collimated AGN winds, and Figures 3.34 and 3.35 for the models with AGN winds with $10^{\circ}$ opening angle.

In Figure 3.32, which shows the models with collimated AGN winds at $t=0.225 \mathrm{Myr}$, i.e., when the AGN wind crosses from the disk to the halo, we see that the presence of magnetic fields do not influence much these diagrams. This indicates that the amount of gas at given densities and temperatures is nearly the same at a given velocity in these models. The only notable difference is the appearance of structures in the velocitytemperature diagram of the model MHD-SN Ia-SN II-AGN $0^{\circ}-B_{x}$ (see middle, bottom diagram of Figure 3.32), with velocity $\sim 300 \mathrm{~km} \mathrm{~s}^{-1}$ and temperature $\sim 10^{4} \mathrm{~K}$. All the diagrams indicate that at this stage of the evolution, the maximum velocities are around $10^{4} \mathrm{~km} \mathrm{~s}^{-1}$ and the structures attaining these velocities are very hot with $\mathrm{T}>10^{7} \mathrm{~K}$, and densities below $0.1 \mathrm{~cm}^{-3}$. They could in principle explain the features observed in UFOs, but not those observed in the molecular outflows which require temperatures smaller than 

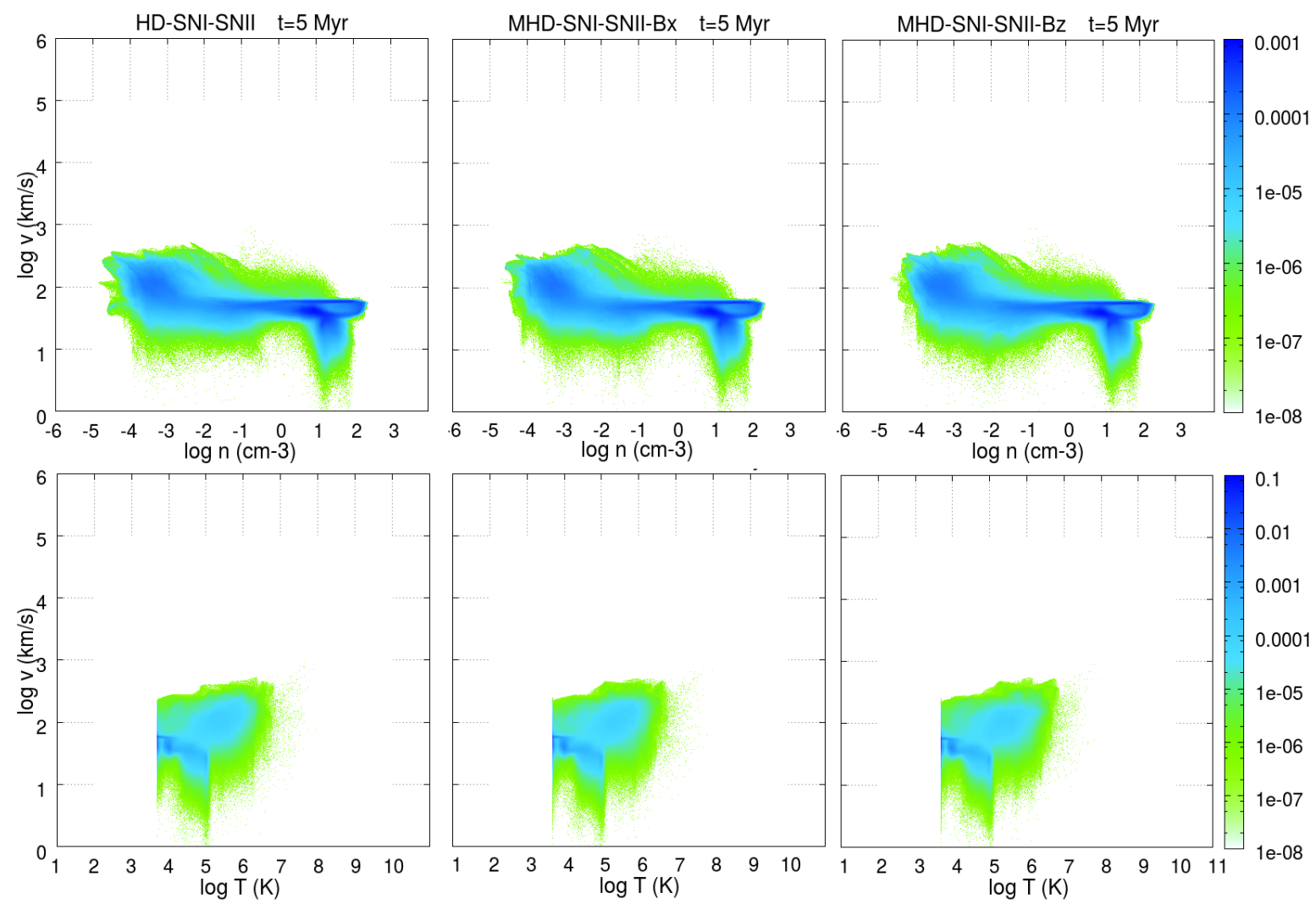

Figure 3.31: Two dimensional histograms of the vertical velocity versus density (V-n) (top), and velocity vs. temperature (V-T)(bottom) calculated considering every cell within the whole simulated system at $t=5 \mathrm{Myr}$, for the three models with SF-wind only, from left to right: the HD model, the model with initial horizontal magnetic field, and the model with initial vertical field, both with $\beta=300$. Velocity is in $\mathrm{km} \mathrm{s}^{-1}$, density in $\mathrm{cm}^{-3}$ and temperature in $\mathrm{K}$, all in logarithmic scale. The color bar indicates the cell number normalized to their total number. The names of the models are indicated at the top of each panel.

$1000 \mathrm{~K}$.

Figure 3.33 shows the same models as in Figure 3.32, but at later time $t=5$ Myr. We see that more gas is accelerated to velocities around $10^{4} \mathrm{~km} \mathrm{~s}^{-1}$ with densities up to $\sim 1 \mathrm{~cm}^{-3}$ and temperatures $>10^{5} \mathrm{~K}$, specially in the magnetized models which allow for more accelerated structures than the HD counterpart, as seen before. As in the earlier phase, we also see structures with velocities of a few $1000 \mathrm{~km} \mathrm{~s}^{-1}$ in all these diagrams, but the correspoding densities are too low and the temperatures too high to explain the observed molecular outflows, as described in the Chapter 1.

Figures 3.34 and 3.35, which show the histograms for the models with AGN wind with $10^{\circ}$ opening angle at $t=0.225$ and $5 \mathrm{Myr}$, indicate that these accelerate more gas to higher speeds, for the same ranges of temperatures and densities, than the models with collimated AGN winds, as one might expect. While in the early evolution, we also see no significant differences between the magnetic and the HD models, at $t=5 \mathrm{Myr}$, we clearly see that the MHD models speed up more dense structures than the HD counterpart. Again, we identify low density structures with maximum velocities as high as $\sim 10^{4} \mathrm{~km}$ 

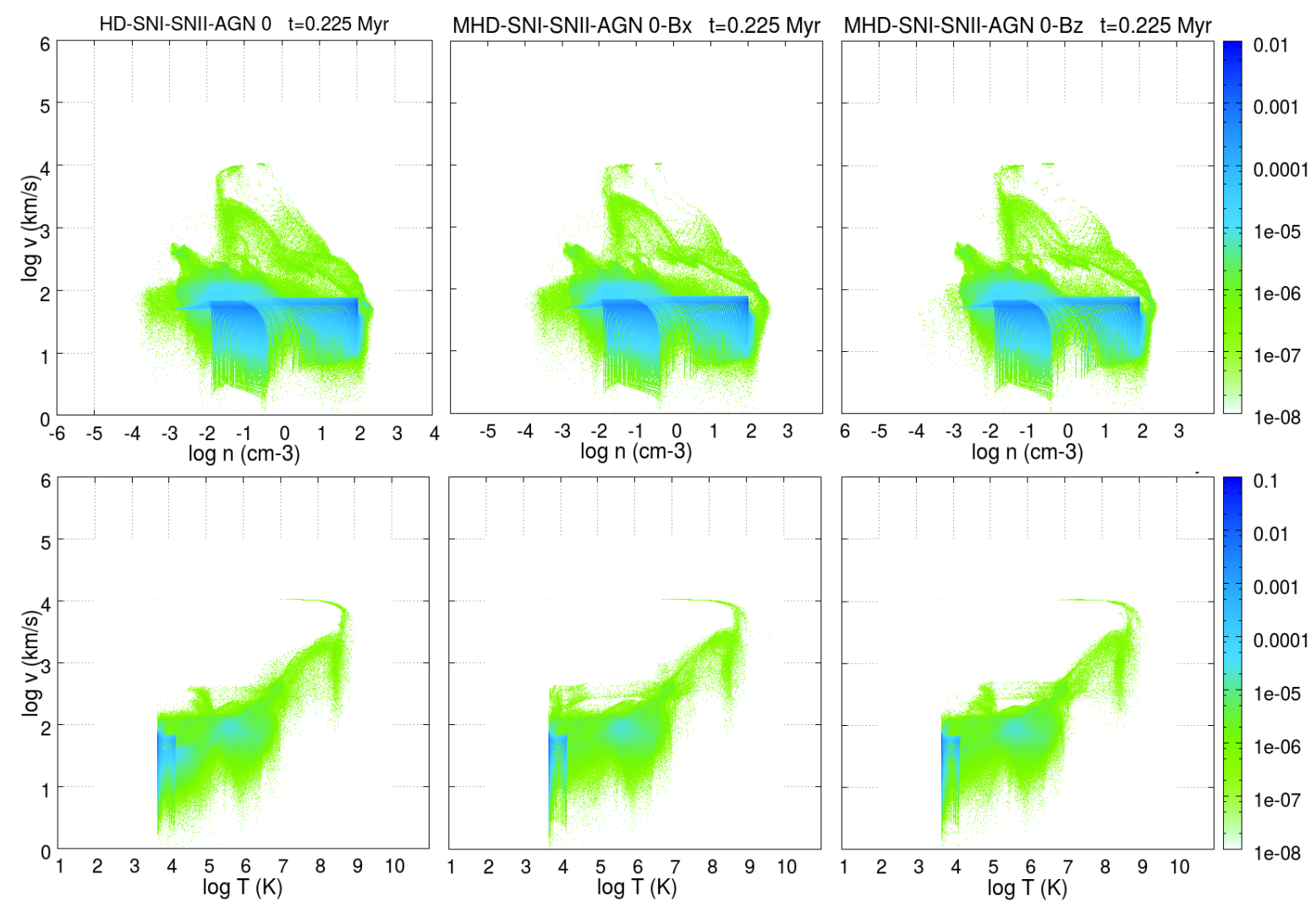

Figure 3.32: Two dimensional histograms of the vertical velocity versus density (V-n) (top), and velocity vs. temperature (V-T)(bottom) calculated considering every cell within the whole simulated system at $t=0.225 \mathrm{Myr}$, for the three models with collimated AGN-wind, from left to right: the HD model, the model with initial horizontal magnetic field, and the model with initial vertical field, both with $\beta=300$. Velocity is in $\mathrm{km} \mathrm{s}^{-1}$, density in $\mathrm{cm}^{-3}$ and temperature in $\mathrm{K}$, all in logarithmic scale. The color bar indicates the cell number normalized to their total number. The names of the models are indicated at the top of each panel.

$\mathrm{s}^{-1}$ and $\mathrm{T} \gg 10^{4} \mathrm{~K}$, as expected for UFOs, and structures with intermediate velocities of several $\sim 100 \mathrm{~km} \mathrm{~s}^{-1}$ with densities up to a few $10 \mathrm{~cm}^{-3}$, as required by the molecular outflows, but the corresponding temperatures for these structures are too high $(>1000$ $\mathrm{K})$ to explain the observed molecular features.

This situation does not change much when we consider models with smaller values of initial $\beta$ (and thus larger initial magnetic fields), as in Figure 3.36, where we have plotted the 2D histograms for the MHD model with initial horizontal field and AGN wind with $10^{\circ}$ opening angle, considering two values of initial $\beta=300$ (as in Figure 3.35), and 30. As we see in this figure, the two histograms are very similar, both in the velocity-density and in the velocity-temperature distributions, except for the fact that a decreasing $\beta$ (larger magnetic field) enlarges a little the amount of gas at higher speeds, attaining maximum values of a few $10^{4} \mathrm{~km} \mathrm{~s}^{-1}$. This result is compatible with those of Figure 3.36 for $\beta=30$, which revealed similar morphological features to those of Figure the model with $\beta=300$. We note that we have also performed simulations of MHD models with collimated AGN winds, considering different initial values of $\beta=30$, and 3 , and the results found are 

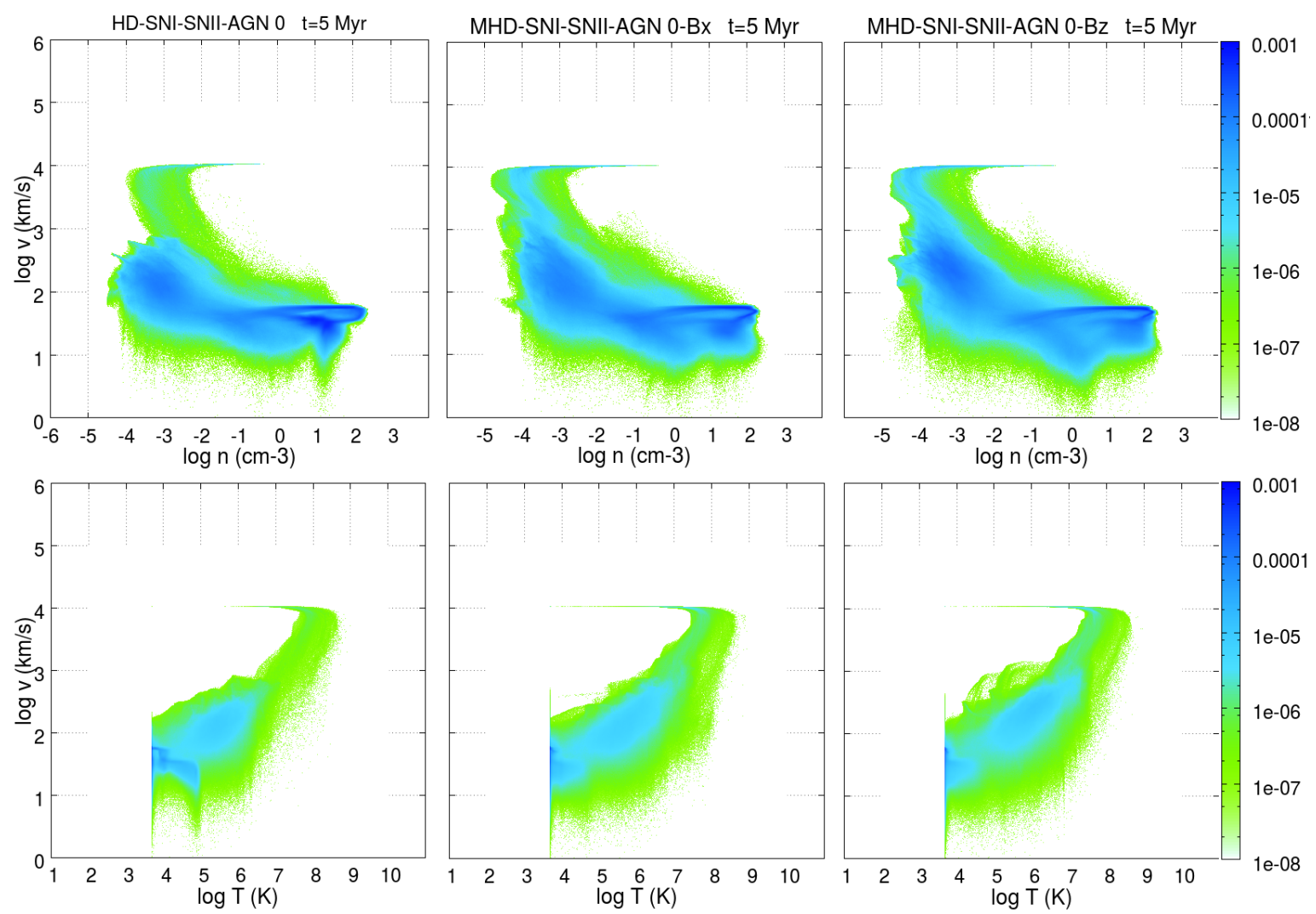

Figure 3.33: Two dimensional histograms of the vertical velocity versus density (V-n) (top), and velocity vs. temperature (V-T) (bottom) calculated considering every cell within the whole simulated system at $t=5 \mathrm{Myr}$, for the three models with collimated AGN-wind, from left to right: the HD model, the model with initial horizontal magnetic field, and the model with initial vertical field, both with $\beta=300$. Velocity is in $\mathrm{km} \mathrm{s}^{-1}$, density in $\mathrm{cm}^{-3}$ and temperature in $\mathrm{K}$, all in logarithmic scale. The color bar indicates the cell number normalized to their total number. The names of the models are indicated at the top of each panel.

similar to those above.

The results of this section can be summarized as follows:

- Outflows with maximum velocities of $\sim 1000 \mathrm{~km} \mathrm{~s}^{-1}$ can be driven by SF-driven winds alone, but the corresponding temperatures of the structures are too large compared to observed molecular or HI outflows, as described in Chapter 1.

- Models that include AGN winds with a larger opening angle and magnetic fields lead to much larger average speeds than models with no AGN wind, and also allow for more gas to be accelerated to maximum velocities around $\sim 10^{4} \mathrm{~km} \mathrm{~s}^{-1}$ (than models with collimated AGN winds), with densities and temperatures which are compatible with those observed in UFOs, but the structures with intermediate velocities of several $\sim 100 \mathrm{~km} \mathrm{~s}^{-1}$ and densities up to a few $10 \mathrm{~cm}^{-3}$, as required by the molecular outflows, also have temperatures too high to explain the observed molecular features. 

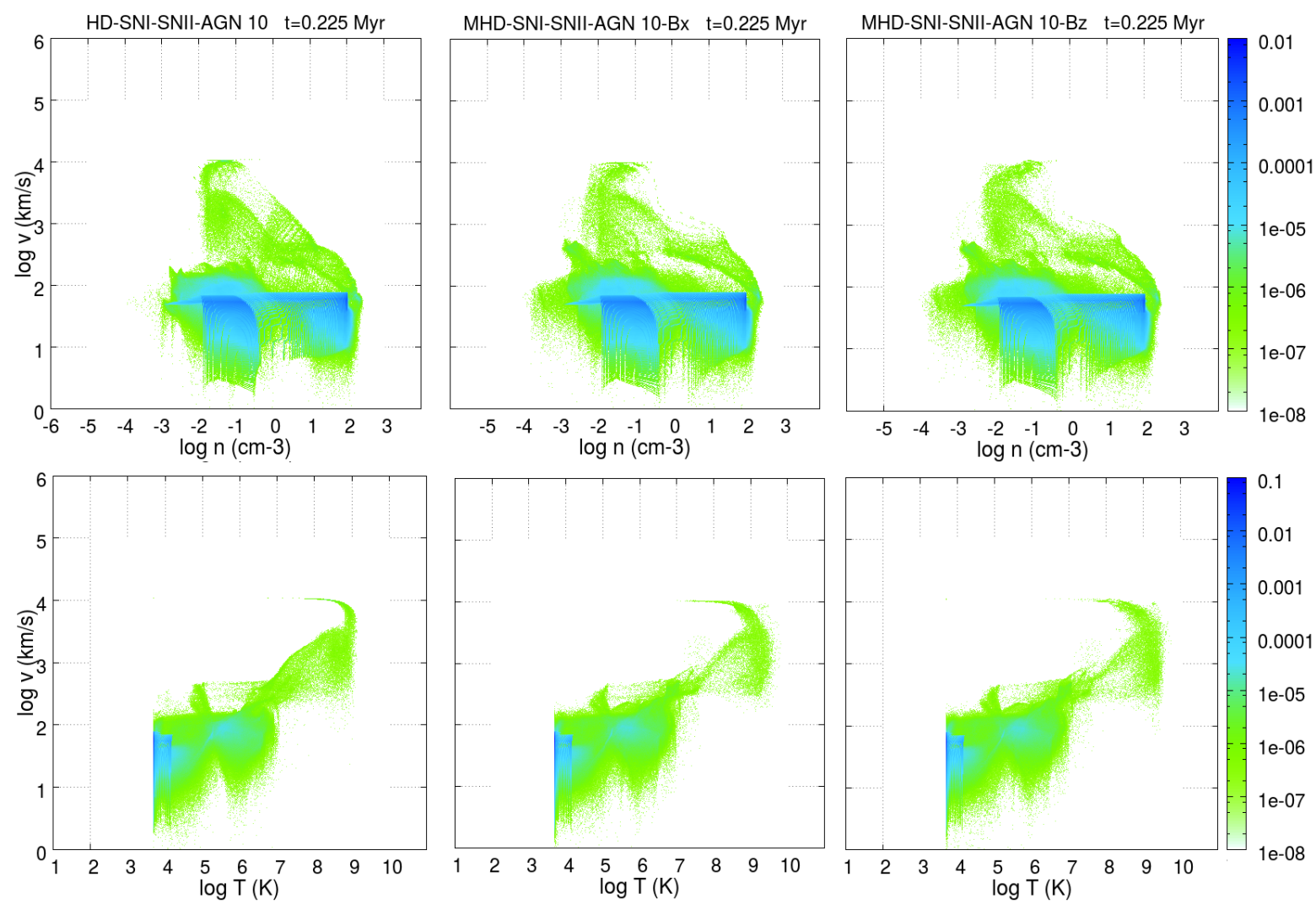

Figure 3.34: Two dimensional histograms of the vertical velocity vs. density (V-n)(top) and velocity vs. temperature (V-T)(bottom)at $t=0.225 \mathrm{Myr}$, for the models with AGN-wind with $10^{\circ}$ opening angle, from left to right: the HD model, the model with initial horizontal magnetic field, and the model with initial vertical field, both with $\beta=300$. Velocity is in $\mathrm{km} \mathrm{s}^{-1}$, density in $\mathrm{cm}^{-3}$ and temperature in $\mathrm{K}$, all in logarithmic scale. The color bar indicates the cell number normalized to their total number. The names of the models are indicated at the top of each panel 

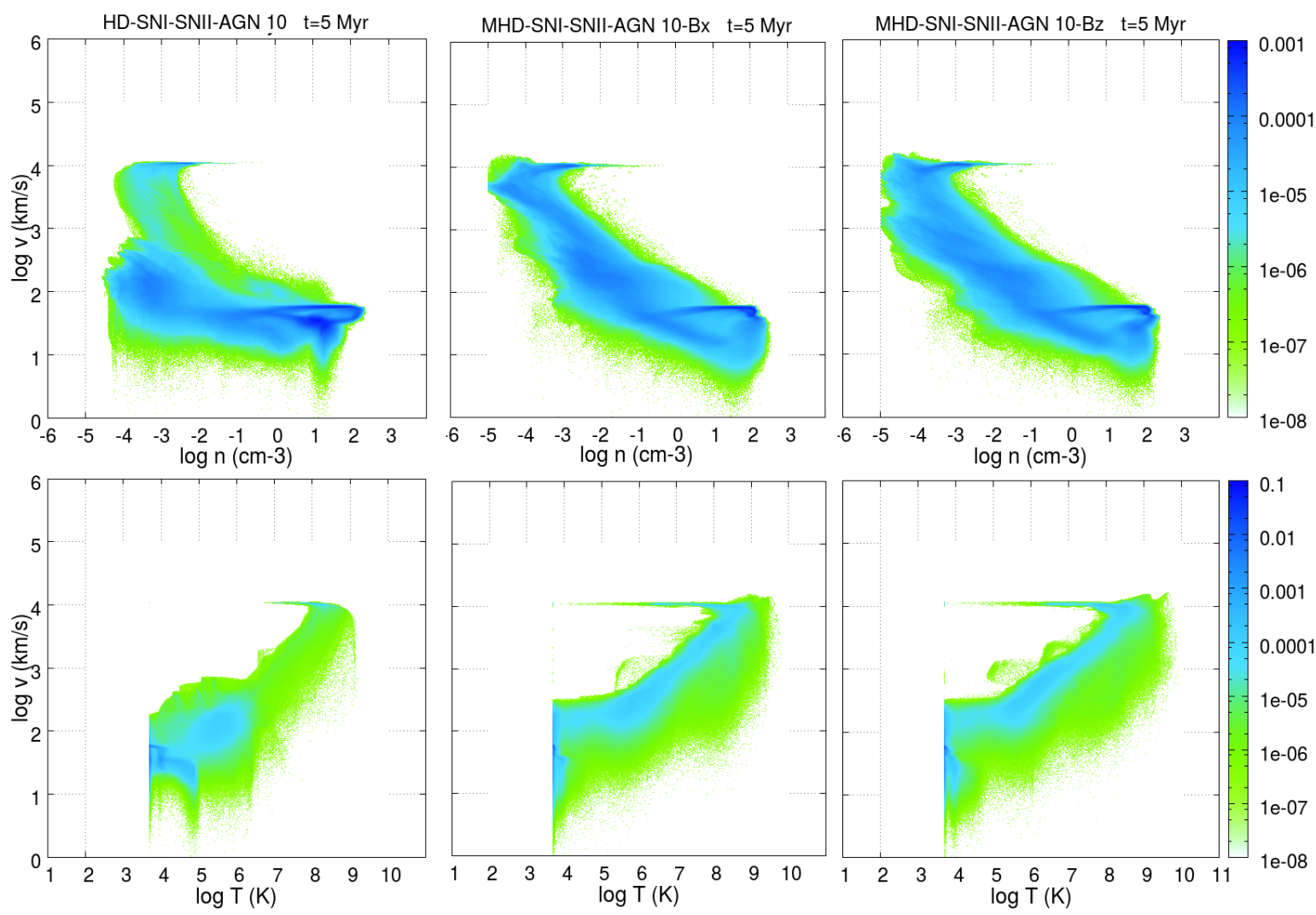

Figure 3.35: Two dimensional histograms of the vertical velocity vs. density (V-n)(top) and velocity vs. temperature (V-T)(bottom)at $t=5 \mathrm{Myr}$, for the models with AGN-wind with $10^{\circ}$ opening angle, from left to right: the HD model, the model with initial horizontal magnetic field, and the model with initial vertical field, both with $\beta=300$. Velocity is in $\mathrm{km} \mathrm{s}^{-1}$, density in $\mathrm{cm}^{-3}$ and temperature in $\mathrm{K}$, all in logarithmic scale. The color bar indicates the cell number normalized to their total number. The names of the models are indicated at the top of each panel 

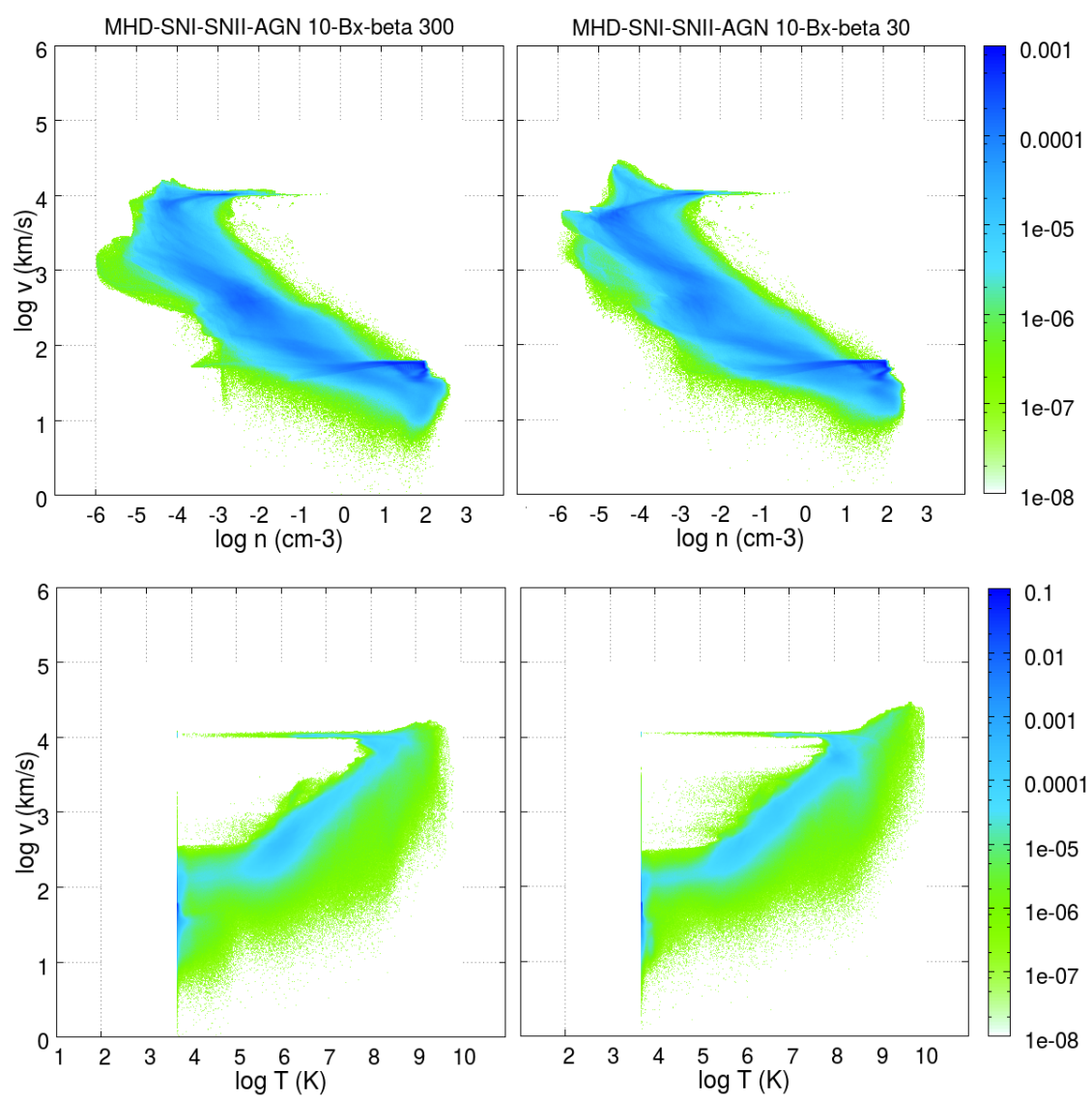

Figure 3.36: Two dimensional histograms of the vertical velocity versus density $(V-n)$, and versus temperature $(\mathrm{V}-\mathrm{T})$ at $t=5 \mathrm{Myr}$, for the models with $\mathrm{AGN}$-wind with $10^{\circ}$ opening angle, nd initial horizontal magnetic field with for two values of $\beta=300$ (left panel), and 30 (right panel). Velocity is in $\mathrm{km} \mathrm{s}^{-1}$, density in $\mathrm{cm}^{-3}$ and temperature in $\mathrm{K}$, all in logarithmic scale. The color bar indicates the cell number normalized to their total number. The names of the models are indicated at the top of each panel. 

CHAPTER 4

\section{Conclusions and Perspectives}

\subsection{Conclusions}

In spite of the observational and theoretical advances in recent years, the processes that drive outflows in active galaxies (AGNs), like the molecular and HI outflows and the ultra-fast outflows (UFOs) (see e.g., Morganti et al. 2013, 2014, 2015; Tombesi et al. 2011, 2013; Kraemer et al. 2018), are still a matter of debate. There is general consensus that the supermassive black hole (SMBH) in the nuclear region of these galaxies has a key role in driving these outflows, but the products of star-formation (SF), like the supernova explosions may also drive starburst winds that can also play some role in driving these outflows (Melioli \& de Gouveia Dal Pino 2015). Particularly in Seyfert galaxies and ULIRGs, where starbursts and star formation regions coexist with the AGN SMBH, and where the AGN emits an amount of energy comparable to that of the star formation regions of the galaxy.

In this dissertation, we have extended the previous numerical studies performed by Melioli \& de Gouveia Dal Pino (2015) on the origin of the outflows in Seyfert galaxies, by adding new physical ingredients. In order to achieve a better understanding of the processes driving the nuclear gas evolution in a Seyfert galaxy, we have performed fully 3D radiative cooling hydrodynamical (HD) and magnetohydrodynamical (MHD) simulations, taking into account the mechanical feedback of both the AGN SMBH and the SF, as performed in previous studies (Melioli \& de Gouveia Dal Pino 2015; Wagner et al. 2012), but have also included the effects of magnetic fields, which were not included in these previous studies, and of AGN winds with a large opening angle. 
We investigated, by means of these 3D MHD simulations, the formation of outflows considering the galaxy evolution for 11 different models with different initial conditions, as described in Table 2.2, adopting magnetic fields with two different initial geometries (a horizontal and a vertical magnetic field to the galactic disc) and initial ratios between the thermal and the magnetic pressures, $\beta=300$, and 30 (implying initial magnetic fields $=$ $0.76 \mu \mathrm{G}$, and $2.40 \mu \mathrm{G}$, respectively), and two different opening-angles for the AGN wind, $0^{\circ}$ and $10^{\circ}$. The main results of this work can be summarized as follows (see also Chapter $3)$ :

- The winds driven by the products of SF (i.e., by the $\mathrm{SNe}$ ) alone can produce outflows with velocities $\sim 100-1000 \mathrm{~km} \mathrm{~s}^{-1}$, mass outflow rates of the order of $50 \mathrm{M}_{\odot}$ $\mathrm{yr}^{-1}$, densities of $1-10 \mathrm{~cm}^{-3}$, and temperatures between $10^{4}$ and $10^{5} \mathrm{~K}$, which resemble the properties of warm absorbers (WAs; Tombesi et al. 2013) and are also compatible with the velocities of the observed molecular outflows (e.g. Morganti et al. 2013), and with the results found by Melioli \& de Gouveia Dal Pino (2015). However, the obtained densities from the simulations are too small and the temperatures too large to explain the observed values in molecular outflows (which have $\mathrm{n} \sim 150-300 \mathrm{~cm}^{-3}$ and $\left.\mathrm{T}<1000\right)$.

- Collimated AGN winds alone (without the presence of SF-winds) are unable to drive massive ouflows like those observed in molecular and HI outflows, but they can accelerate structures to very high speeds, of the order of $\sim 10.000 \mathrm{~km} \mathrm{~s}^{-1}$, and temperatures $T>10^{6} \mathrm{~K}$ as observed in UFOs (e.g., Tombesi et al. 2011, 2013, 2015). This is also in agreement with previous results of Melioli \& de Gouveia Dal Pino (2015).

- The introduction of an AGN, particularly with a large opening angle, causes the formation of a fountain-like structure at the highest altitudes above the galaxy disc. This makes part of the expanding gas (pushed by the SF-wind) to fallback in the galaxy. This produces a 'positive' feedback on the host galaxy evolution, similar to that detected in earlier studies of galactic fountains (see e.g., Melioli et al. 2008, 2009). On the other hand, this fallback causes a decrease in the mass loss rate in the outflows (by a factor up to 10). We have found that these effects are more pronounced in models that have magnetic fields, due to the action of extra magnetic forces by the AGN Wind.

- The presence of the magnetic fields in the AGN and SF winds combined clearly affects their overall evolution. We find that the propagation velocity of the SFdriven wind and their structures is slightly smaller than in the HD counterpart due to the aditional magnetic pressure support. The AGN wind that blows out into 
a fountain at the highest altitudes refrains the expansion and acceleration of the SF-driven outflow retaining more gas at lower altitudes than in the HD models.

- In the presence of magnetic fields, part of the energy of the shocks is transferred to the magnetic field, decreasing the strength of the shocks and preserving more the clump structures from disruption.

- The presence of a collimated AGN wind $\left(0^{\circ}\right)$ causes a significant removal of mass from the core region in a few $100.000 \mathrm{yr}$, but this is soon replenished by gas inflow from the interstellar medium (ISM) when the SNe explosions fully develop. On the other hand, an AGN wind with a large opening angle in presence of magnetic fields is able to remove the nuclear gas entirely within a few $100.000 \mathrm{yr}$ and does not allow for later replenishment. Therefore, it quenches the fueling and mass accretion onto the SMBH. This indicates that the duty cycle of these outflows is around a few $100.000 \mathrm{yr}$, compatible with the timescales inferred for the observed UFOs and molecular outflows.

- To summarize, models that include AGN winds with a larger opening angle and magnetic fields lead to much larger average speeds than models with no AGN wind, and also allow for more gas to be accelerated to maximum velocities around $\sim 10^{4}$ $\mathrm{km} \mathrm{s}^{-1}$ (than models with collimated AGN winds), with densities and temperatures wich are compatible with those observed in UFOs. However, the structures with intermediate velocities of several $\sim 100 \mathrm{~km} \mathrm{~s}^{-1}$ and densities up to a few $100 \mathrm{~cm}^{-3}$, that in fact could reproduce the observed molecular outflows, have temperatures wich are too large to explain the observed molecular features, wich have temperatures between T 100 and $<1000 \mathrm{~K}$ (see, Morganti et al. 2013, Thadhunter et al., 2014, Kraemer et al., 2018). Furthermore, these large opening angle AGN winds with magnetic fields reduce the mass loss rates of the outflows to values smaller than those observed both in molecular outflows and UFOs.

\subsection{Perspectives for future work}

Several features observed in active galaxy outflows and feedback were reproduced in our simulated models like, for instance, the typical times of the duty cycle of the UFOs and molecular outflows, and the high-speed velocities and temperatures observed in the UFOs. However, important features, as the observed temperatures and densities in molecular outflows structures were not reproduced. As remarked before, molecular outflows with temperatures of the order of $\sim 10^{2} \mathrm{~K}$ or lower, and densities of the order of $10^{2} \mathrm{~cm}^{-3}$ are observed interacting co-spatially with outflowing jets at kpc scales. In our simulations the temperatures are larger (and the densities are generally smaller) than 
those observed in the molecular outflows and possibly some important ingredient in our simulations is missing. As remarked, we have performed numerical simulations assuming a radiative cooling of the gas at ionization equilibrium. This means, that we did not allow for the chemical species to have their abundances to vary with time and the temperature of the gas to realistically decrease to very low values typical of molecular species (like the hydrogen gas $\mathrm{H}_{2}$ ), of the order of $1000 \mathrm{~K}$ or less. We did not account either for the presence of dust, which can reach even smaller temperatures. In forthcoming studies, we intend to include the effects of non-equilibrium ionizing radiative cooling, allowing for the formation of very cold clumps and even dusty structures in our simulations. For this aim, we can employ modified versions of algorithms already developed by the group and collaborators (e.g., Melioli, de Gouveia Dal Pino and Raga 2004).

Also, we expect that higher intensity magnetic fields can shield cold dense structures against the disruption and evaporation by the impinging outflows driven by the AGN and the SNe. Therefore, we intend to expand the parametric space investigated in this work, to study the effects of magnetic fields with higher intensities through the $\beta$ relation and more complex initial geometries, like the inclusion of a helical structure in the AGN wind component.

Finally, weakly collimated outflows are commonly observed, implying a wide interaction between the AGN-wind and the ISM. This motivates us to include in our future work a broader parametric space sweeping several opening angles of the AGN-wind, in order to explore how this geometric parameter is coupled with the ISM and even more, with the magnetic field since as we saw before, the magnetic fields in tandem with conical AGNwind produce substantial effects on the feedback at the galaxy, like the drop in fueling to the nuclear region and the increase of the acceleration of the gas. 


\section{The Amun Code}

The AMUN CODE is a three-dimensional code which solves the MHD equations in the following form, assuming implicit equilibrium radiative cooling of the gas:

$$
\frac{\partial \mathbf{U}}{\partial t}+\frac{\partial \mathbf{F}}{\partial x}+\frac{\partial \mathbf{G}}{\partial y}+\frac{\partial \mathbf{H}}{\partial z}=\mathbf{S}
$$

where $\mathbf{U}, \mathbf{F}, \mathbf{G}, \mathbf{H}$ and $\mathbf{S}$ are given by,

$$
\mathbf{U}=\left[\begin{array}{c}
\rho \\
m_{x} \\
m_{y} \\
m_{z} \\
E \\
B_{x} \\
B_{y} \\
B_{z}
\end{array}\right]
$$




$$
\begin{aligned}
& \mathbf{F}=\left[\begin{array}{c}
\rho v_{x} \\
\rho v_{x}^{2}+p+B^{2} / 2-B_{x}^{2} \\
\rho v_{x} v_{y}-B_{x} B_{y} \\
\rho v_{x} v_{z}-B_{x} B_{z} \\
\left(E+p^{*}\right) v_{x}-(\mathbf{B} \cdot \mathbf{v}) B_{x} \\
0 \\
B_{y} v_{x}-B_{x} v_{y} \\
B_{z} v_{x}-B_{x} v_{z}
\end{array}\right] \\
& \mathbf{G}=\left[\begin{array}{c}
\rho v_{y} \\
\rho v_{y} v_{x}-B_{y} B_{x} \\
\rho v_{y}^{2}+p+B^{2} / 2-B_{y}^{2} \\
\rho v_{y} v_{z}-B_{y} B_{z} \\
\left(E+p^{*}\right) v_{y}-(\mathbf{B} \cdot \mathbf{v}) B_{y} \\
B_{x} v_{y}-B_{y} v_{x} \\
0 \\
B_{z} v_{y}-B_{y} v_{z}
\end{array}\right] \\
& \mathbf{H}=\left[\begin{array}{c}
\rho v_{z} \\
\rho v_{z} v_{x}-B_{z} B_{x} \\
\rho v_{z} v_{y}-B_{z} B_{y} \\
\rho v_{z}^{2}+p+B^{2} / 2-B_{z}^{2} \\
\left(E+p^{*}\right) v_{z}-(\mathbf{B} \cdot \mathbf{v}) B_{z} \\
B_{x} v_{z}-B_{z} v_{x} \\
B_{y} v_{z}-B_{z} v_{y} \\
0
\end{array}\right] \\
& \mathbf{S}=\left[\begin{array}{c}
\dot{m}_{s} \\
\dot{m}_{s} v_{x}+f_{G x} \\
\dot{m}_{s} v_{y}+f_{G y} \\
\dot{m}_{s} v_{z}+f_{G z} \\
E_{S N I a}+E_{S N I I}+E_{A G N}+E_{D M}+E_{\text {disk }}+E_{\text {bulge }}-\Lambda(\rho, T) \\
0 \\
0 \\
0
\end{array}\right]
\end{aligned}
$$

where $\mathbf{m}=\rho \mathbf{v}$ is the momentum density, $v_{x}, v_{y}, v_{z}$ are the velocity components, $B_{x}, B_{y}, B_{Z}$ are the magnetic field components, $p$ is the pressure, $E$ the internal energy and $p *=$ $p+B^{2} / 2$. The matrix $\mathbf{S}$ represents the source terms in our problem: $\dot{m}_{s}$ represents the mass injection by SN Ia, SN II and the AGN-wind per unit volume; $E_{S N I a}, E_{S N I I}$ and 

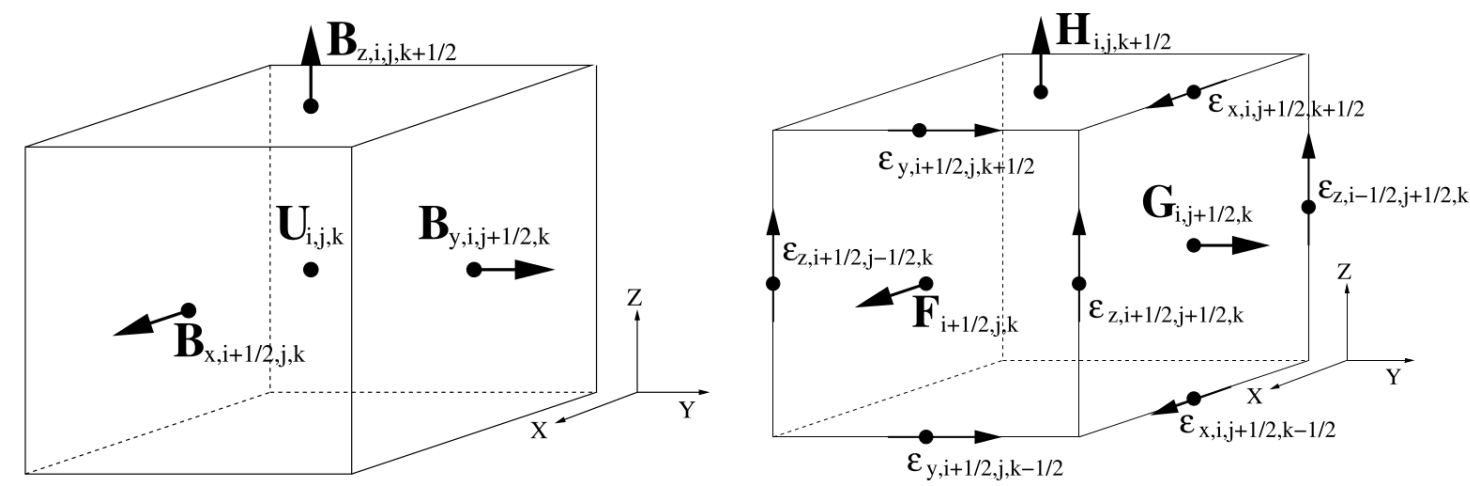

Figure A.1: Centering of volume-averaged conserved variables $U$ and area-averaged components of magnetic field B on the grid. Right: Centering of time- and area-averaged components of the fluxes of $\mathrm{U}$ on the grid (picture taken from Stone 2008).

$E_{A G N}$ the energy injection by SN Ia, SN II and the AGN-wind per unit volume and $\Lambda(\rho, T)$ is the energy loss by radiative cooling. The AMUN code integrates equations on a regular 3D cartesian grid (Figure A.1).

The spatial coordinates $(x, y, z)$ are discretized into $\left(N_{x}, N_{y}, N_{z}\right)$ cells in a domain with dimensions $L_{x}, L_{y}, L_{z}$ in each direction, respectively. The cell $(i, j, k)$ is centered at the position $\left(x_{i}, y_{j}, z_{k}\right)$. The width of each cell, $\Delta x=L_{x} / N_{x}, \Delta y=L_{y} / N_{y}, \Delta z=L_{z} / N_{z}$ is constant along all the computational domain. The discretization of the time steps can be variable, so the convention for the time interval $\Delta t^{n}=t^{n+1}-t^{n}$ is substitued by $\Delta t$ to say that the time interval can change due to the Courant criteria which is based on the propagation velocity of information through the cells. ${ }^{1}$ Here, half-integer subscripts denote the edges of the computational cells, that is, $x_{i-1 / 2}$ is the location of the interface between the cells centered at $x_{i-1}$ and $x_{i}$. Thus the fluxes $\mathbf{F}, \mathbf{G}$ and $\mathbf{H}$ (eqs. 4.3-4.5), are evaluated at the faces of each grid cell. On the other hand, the half-integer superscript on the fluxes denote a time average, rather than representing the flux evaluated at $t^{n+1 / 2}$.

The integration of the equation of continuity, momentum and energy is based in the discretization based in the integral rather than in the differential form because this represents several advantages at the moment to calculate shocks and discontinuities (Le Veque 2002). The integration of the magnetic flux is based on the constraint $\nabla \cdot \mathbf{B}=0$, as we shall see.

The integration of equation A.1 over a discrete time interval $\Delta t$ and using the divergence theorem produces,

\footnotetext{
${ }^{1}$ This criteria is used to calculate the next time step taking into account that the velocity propagation of the information in the fluid, i.e., the sound speed (or the Alfven speed if this is larger than sound speed) in the fluid, is small enough to not exeed the longitudinal size $\Delta x_{i}$ of a given cell.
} 


$$
\begin{aligned}
\mathbf{U}_{i, j, k}^{n+1 / 2}=\mathbf{U}_{i, j, k}^{n} & -\frac{\Delta t}{\Delta x}\left(\mathbf{F}_{i+1 / 2, j, k}^{n+1 / 2}-\mathbf{F}_{i-1 / 2, j, k}^{n+1 / 2}\right) \\
& -\frac{\Delta t}{\Delta y}\left(\mathbf{G}_{i, j+1 / 2, k}^{n+1 / 2}-\mathbf{G}_{i, j-1 / 2, k}^{n+1 / 2}\right) \\
& -\frac{\Delta t}{\Delta z}\left(\mathbf{H}_{i, j, k+1 / 2}^{n+1 / 2}-\mathbf{G}_{i, j, k-1 / 2}^{n+1 / 2}\right)
\end{aligned}
$$

where

$$
\mathbf{U}_{i, j, k}^{n}=\frac{1}{\Delta x \Delta y \Delta z} \int_{z_{k-1 / 2}}^{z_{k+1 / 2}} \int_{y_{j-1 / 2}}^{y_{j+1 / 2}} \int_{x_{i-1 / 2}}^{x_{i+1 / 2}} \mathbf{U}\left(x, y, z, t^{n}\right) d x d y d z,
$$

is a vector of volume-averaged variables, while

$$
\begin{aligned}
& \mathbf{F}_{i-1 / 2, j, k}^{n+1 / 2}=\frac{1}{\Delta y \Delta z \Delta t} \int_{t^{n}}^{t^{n+1}} \int_{z_{k-1 / 2}}^{z_{k+1 / 2}} \int_{y_{j-1 / 2}}^{y_{j+1 / 2}} \mathbf{F}\left(x_{i-1 / 2}, y, z, t\right) d y d z d t \\
& \mathbf{G}_{i, j-1 / 2, k}^{n+1 / 2}=\frac{1}{\Delta x \Delta z \Delta t} \int_{t^{n}}^{t^{n+1}} \int_{x_{i-1 / 2}}^{x_{i+1 / 2}} \int_{z_{k-1 / 2}}^{z_{k+1 / 2}} \mathbf{G}\left(x, y_{j-1 / 2}, z, t\right) d x d z d t, \\
& \mathbf{H}_{i, j, k-1 / 2}^{n+1 / 2}=\frac{1}{\Delta x \Delta y \Delta t} \int_{t^{n}}^{t^{n+1}} \int_{y_{j-1 / 2}}^{y_{j+1 / 2}} \int_{x_{i-1 / 2}}^{x_{i+1 / 2}} \mathbf{H}\left(x, y, z_{k-1 / 2}, t\right) d x d y d t,
\end{aligned}
$$

are vectors of the time and area averged fluxes. This set of equations is evolved using a second-order Runge-Kutta scheme for time integration and the Harten-Lax-van Leer C solver for the time averaged fluxes (see Toro 1999 for an introduction to the subject). The radiative energy losses $\Lambda(\rho, T)=n^{2} P_{r a d}$, where $n$ is the density and $P_{\text {rad }}$ is the energy radiated per second, are calculated implicitly curves given by optically thin gas in ionization equlibrium using the radiative cooling curves given by $\tilde{\Lambda}$ by Hollenbach \& McKee 1989, McWhirter et al. (1975) (see Figure A.2).

The magnetic field components are stored at the center of the faces of the cells. The divergence of the magnetic vector field is given by,

$$
\begin{aligned}
(\nabla \cdot \vec{B})_{i, j, k} & =\frac{B_{i+1 / 2, j, k}^{x}-B_{i-1 / 2, j, k}^{x}}{\Delta x} \\
& =\frac{B_{i, j+1 / 2, k}^{y}-B_{i, j-1 / 2, k}^{y}}{\Delta y} \\
& =\frac{B_{i, j, k+1 / 2}^{z}-B_{i, j, k-1 / 2}^{z}}{\Delta z} .
\end{aligned}
$$

This constraint is keep null (to the machine precision) using the constrained trans- 


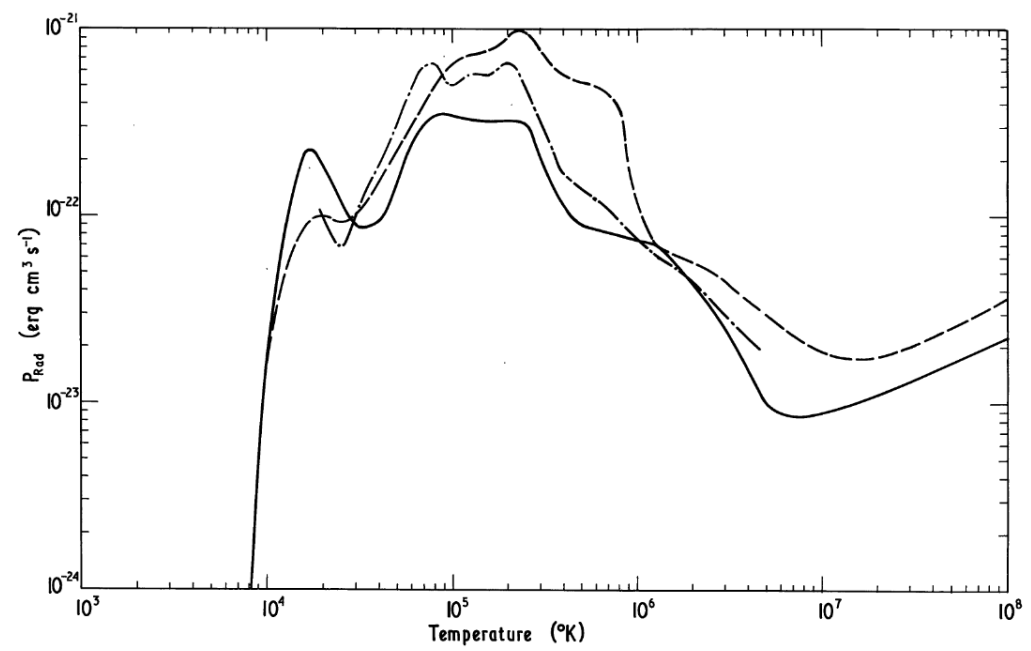

Figure A.2: Radiative cooling curve $P_{\text {rad }}$ for opticaly thin gas with radiative looses by: Bremsstrahlung, recombination and emission lines. The radiative cooling rate (eq. A6) is given by $\Lambda=n^{2} P_{\text {rad }}$ erg $\mathrm{cm}^{-3} \mathrm{~s}^{-1}$. The continuous line was calculated by McWhirter et al. (1975) for the solar corona. For comparasion, Pottasch (1965) (chain line) and Cox and Tucker (1969) (dashed line).

port method (CT) (Evans \& Hawley 1988). After this, the value of the magnetic field components in the center of each one of the cells are given by the second order accurate averages,

$$
\begin{aligned}
B_{i, j, k}^{x} & =\frac{1}{2}\left(B_{i+1 / 2, j, k}^{x}+B_{i-1 / 2, j, k}^{x}\right) \\
B_{i, j, k}^{y} & =\frac{1}{2}\left(B_{i, j+1 / 2, k}^{y}+B_{i, j-1 / 2, k}^{y}\right) \\
B_{i, j, k}^{z} & =\frac{1}{2}\left(B_{i, j, k+1 / 2}^{z}+B_{i, j, k-1 / 2}^{z}\right)
\end{aligned}
$$




\section{BIBLIOGRAPHY}

Aalto, S., Muller, S., Sakamoto, K., Gallagher, J. S., Martín, S., and Costagliola, F. Winds of change - a molecular outflow in NGC 1377?. The anatomy of an extreme FIR-excess galaxy. aap, 546:A68, October 2012. doi: 10.1051/0004-6361/201118052.

Armus, L., Heckman, T., and Miley, G. Multicolor optical imaging of powerful far-infrared galaxies - More evidence for a link between galaxy mergers and far-infrared emission. aj, 94:831-846, October 1987. doi: 10.1086/114517.

Beck, R., de Gouveia dal Pino E. M., Lugones, G., and Lazarian, A., editors. Magnetic Fields in the Universe: From Laboratory and Stars to Primordial Structures, volume 784 of American Institute of Physics Conference Series, September 2005.

Blandford, R. D. and Payne, D. G. Hydromagnetic flows from accretion discs and the production of radio jets. MNRAS, 199:883-903, June 1982. doi: 10.1093/mnras/199.4. 883.

Blandford, R. D. and Znajek, R. L. Electromagnetic extraction of energy from Kerr black holes. MNRAS, 179:433-456, May 1977. doi: 10.1093/mnras/179.3.433.

Carilli, C. L., Dreher, J. W., Conner, S., and Perley, R. A. Broad- and narrow-band imaging of the giant radio galaxy Cygnus A. aj, 98:513-523, August 1989. doi: 10. $1086 / 115155$.

Cicone, C., Maiolino, R., Sturm, E., Graciá-Carpio, J., Feruglio, C., Neri, R., Aalto, S., Davies, R., Fiore, F., Fischer, J., García-Burillo, S., González-Alfonso, E., HaileyDunsheath, S., Piconcelli, E., and Veilleux, S. Massive molecular outflows and evidence 
for AGN feedback from CO observations. Aap, 562:A21, February 2014. doi: 10.1051/ 0004-6361/201322464.

Ciotti, L. AGN feedback in numerical simulations. Highlights of Astronomy, 15:293-293, November 2010. doi: 10.1017/S1743921310009348.

Ciotti, L. and Ostriker, J. P. Cooling Flows and Quasars. II. Detailed Models of Feedbackmodulated Accretion Flows. Apj, 551:131-152, April 2001. doi: 10.1086/320053.

Ciotti, L. and Ostriker, J. P. Radiative Feedback from Massive Black Holes in Elliptical Galaxies: AGN Flaring and Central Starburst Fueled by Recycled Gas. Apj, 665: 1038-1056, August 2007. doi: 10.1086/519833.

Combes, F. Symposium 342, Perseus in Scicily: from black holes to the outskirts of clusters of galaxies, 2018, submitted. Procs. of the IAU, 2018.

Croton, D. J., Springel, V., White, S. D. M., De Lucia, G., Frenk, C. S., Gao, L., Jenkins, A., Kauffmann, G., Navarro, J. F., and Yoshida, N. The many lives of active galactic nuclei: cooling flows, black holes and the luminosities and colours of galaxies. MNRAS, 365:11-28, January 2006. doi: 10.1111/j.1365-2966.2005.09675.x.

Dasyra, K. M. and Combes, F. Cold and warm molecular gas in the outflow of 4C 12.50. aap, 541:L7, May 2012. doi: 10.1051/0004-6361/201219229.

de Gouveia Dal Pino, E., Henrique Sinki Kadowaki, L., Khiali, B., and Bahadur Singh, C. Particle Acceleration and the origin of Very High Energy emission around black holes. IAU General Assembly, 22:2258048, August 2015.

de Gouveia Dal Pino, E. M. Astrophysical jets and outflows. Advances in Space Research, 35:908-924, 2005. doi: 10.1016/j.asr.2005.03.145.

Elvis, M., Lockman, F. J., and Fassnacht, C. An accurate Galactic $\mathrm{N}_{H}$ map of the north ecliptic pole. Apjs, 95:413-417, December 1994. doi: 10.1086/192105.

Fabian, A.C. Observational evidence of active galactic nuclei feedback. Annual Review of Astronomy and Astrophysics, 50(1):455-489, 2012. doi: 10.1146/annurev-astro-081811-125521. URL https://doi.org/10.1146/ annurev-astro-081811-125521.

Falceta-Gonçalves, D., Caproni, A., Abraham, Z., Teixeira, D. M., and de Gouveia Dal Pino, E. M. Precessing Jets and X-ray Bubbles from NGC 1275 (3C 84) in the Perseus Galaxy Cluster: A View from Three-dimensional Numerical Simulations. Apjl, 713: L74-L78, April 2010a. doi: 10.1088/2041-8205/713/1/L74. 
Falceta-Gonçalves, D., de Gouveia Dal Pino, E. M., Gallagher, J. S., and Lazarian, A. Turbulence and the Formation of Filaments, Loops, and Shock Fronts in NGC 1275. Apjl, 708:L57-L60, January 2010b. doi: 10.1088/2041-8205/708/1/L57.

Falomo, R., Pian, E., and Treves, A. An optical view of BL Lacertae objects. 22:73, September 2014. doi: 10.1007/s00159-014-0073-z.

Faucher-Giguère, C.-A. and Quataert, E. The physics of galactic winds driven by active galactic nuclei. MNRAS, 425:605-622, September 2012. doi: 10.1111/j.1365-2966.2012. 21512.x.

Forbes, D. A. and Norris, R. P. Star formation in southern Seyfert galaxies. MNRAS, 300:757-766, November 1998. doi: 10.1046/j.1365-8711.1998.01940.x.

Gaspari, M., Brighenti, F., D'Ercole, A., and Melioli, C. AGN feedback in galaxy groups: the delicate touch of self-regulated outflows. MNRAS, 415:1549-1568, August 2011a. doi: 10.1111/j.1365-2966.2011.18806.x.

Gaspari, M., Melioli, C., Brighenti, F., and D'Ercole, A. The dance of heating and cooling in galaxy clusters: three-dimensional simulations of self-regulated active galactic nuclei outflows. MNRAS, 411:349-372, February 2011b. doi: 10.1111/j.1365-2966.2010.17688. $\mathrm{x}$.

Gaspari, M., Brighenti, F., and Temi, P. Mechanical AGN feedback: controlling the thermodynamical evolution of elliptical galaxies. MNRAS, 424:190-209, July 2012. doi: 10.1111/j.1365-2966.2012.21183.x.

Genzel, R., Lutz, D., and Tacconi, L. Star formation triggered by galaxy collisions. Nature, 395:859-869, October 1998. doi: 10.1038/27597.

Genzel, R., Förster Schreiber, N. M., Rosario, D., Lang, P., Lutz, D., Wisnioski, E., Wuyts, E., Wuyts, S., Bandara, K., Bender, R., Berta, S., Kurk, J., Mendel, J. T., Tacconi, L. J., Wilman, D., Beifiori, A., Brammer, G., Burkert, A., Buschkamp, P., Chan, J., Carollo, C. M., Davies, R., Eisenhauer, F., Fabricius, M., Fossati, M., Kriek, M., Kulkarni, S., Lilly, S. J., Mancini, C., Momcheva, I., Naab, T., Nelson, E. J., Renzini, A., Saglia, R., Sharples, R. M., Sternberg, A., Tacchella, S., and van Dokkum, P. Evidence for Wide-spread Active Galactic Nucleus-driven Outflows in the Most Massive z 1-2 Star-forming Galaxies. Apj, 796:7, November 2014. doi: 10.1088/ 0004-637X/796/1/7.

Geraissate, F. Simulações Numericas Hidrodinamicas Tridimensionais de Ventos Galacticos: Uma Aplicação a Galaxia de Starburst M82. Tesis de Mestrado, Instituto de Astronomia, Geologia e Ciências Atmosfericas, 2010. 
Gofford, J., Reeves, J. N., Tombesi, F., Braito, V., Turner, T. J., Miller, L., and Cappi, M. The Suzaku view of highly ionized outflows in AGN - I. Statistical detection and global absorber properties. MNRAS, 430:60-80, March 2013. doi: 10.1093/mnras/sts481.

Haehnelt, M. G. Momentum transfer by an internal source of ionizing radiation: an important feedback process during galaxy formation? MNRAS, 273:249-256, March 1995. doi: 10.1093/mnras/273.2.249.

Häring, N. and Rix, H.-W. On the Black Hole Mass-Bulge Mass Relation. Apjl, 604: L89-L92, April 2004. doi: 10.1086/383567.

Heckman, T. M., Armus, L., and Miley, G. K. On the nature and implications of starburstdriven galactic superwinds. Apjs, 74:833-868, December 1990. doi: 10.1086/191522.

Hopkins, P. F., Quataert, E., and Murray, N. Self-regulated star formation in galaxies via momentum input from massive stars. MNRAS, 417:950-973, October 2011. doi: 10.1111/j.1365-2966.2011.19306.x.

Hopkins, P. F., Quataert, E., and Murray, N. Stellar feedback in galaxies and the origin of galaxy-scale winds. MNRAS, 421:3522-3537, April 2012. doi: 10.1111/j.1365-2966. 2012.20593.x.

Jones, A. P. and Nuth, J. A. Dust destruction in the ISM: a re-evaluation of dust lifetimes. Aap, 530:A44, June 2011. doi: 10.1051/0004-6361/201014440.

Kadowaki, L. H. S., de Gouveia Dal Pino, E. M., and Stone, J. M. MHD instabilities in accretion disks and their implications in driving fast magnetic reconnection. ArXiv e-prints, March 2018.

Kennicutt, Jr., R. C. The Global Schmidt Law in Star-forming Galaxies. Apj, 498: 541-552, May 1998. doi: 10.1086/305588.

Ketcheson, E. Highly Efficient Strong Stability-Preserving Runge-Kutta Methods with Low-Storage Implementations. 2008.

King, A. Black Holes, Galaxy Formation, and the $\mathrm{M}_{B H^{-}} \sigma$ Relation. Apjl, 596:L27-L29, October 2003. doi: 10.1086/379143.

King, Andrew and Pounds, Ken. Powerful outflows and feedback from active galactic nuclei. Annual Review of Astronomy and Astrophysics, 53(1):115-154, 2015. doi: 10.1146/annurev-astro-082214-122316. URL https://doi.org/10.1146/ annurev-astro-082214-122316.

Kraemer, S. B., Tombesi, F., and Bottorff, M. C. Physical Conditions in Ultra-fast Outflows in AGN. Apj, 852:35, January 2018. doi: 10.3847/1538-4357/aa9ce0. 
Krause, M. Extragalactic Radio Sources as a Piece of the Cosmological Jigsaw. ArXiv e-prints, April 2018.

Krawczynski, H. and Treister, E. Active galactic nuclei: the physics of individual sources and the cosmic history of formation and evolution. Frontiers of Physics, 8:609-629, December 2013. doi: 10.1007/s11467-013-0310-3.

Kuraszkiewicz, J. K., Green, P. J., Crenshaw, D. M., Dunn, J., Forster, K., Vestergaard, M., and Aldcroft, T. L. Emission Line Properties of AGN from a post-COSTAR FOS HST Spectral Atlas. In American Astronomical Society Meeting Abstracts, volume 35 of Bulletin of the American Astronomical Society, page 1297, December 2003.

Lípari, S., Terlevich, R., Díaz, R. J., Taniguchi, Y., Zheng, W., Tsvetanov, Z., Carranza, G., and Dottori, H. Extreme galactic wind and Wolf-Rayet features in infrared mergers and infrared quasi-stellar objects. MNRAS, 340:289-303, March 2003. doi: 10.1046/j. 1365-8711.2003.06309.x.

Lister, M. The MOJAVE Program: Investigating the Parsec-Scale Evolution of Relativistic Jets in Active Galaxies. In American Astronomical Society Meeting Abstracts, volume 35 of Bulletin of the American Astronomical Society, page 1246, December 2003.

Maiolino, R., Krabbe, A., Thatte, N., and Genzel, R. Seyfert Activity and Nuclear Star Formation in the Circinus Galaxy. Apj, 493:650-665, January 1998. doi: 10.1086/ 305150.

Martin, C. L. Mapping Large-Scale Gaseous Outflows in Ultraluminous Galaxies with Keck II ESI Spectra: Variations in Outflow Velocity with Galactic Mass. Apj, 621: 227-245, March 2005. doi: 10.1086/427277.

McKee, C. Dust Destruction in the Interstellar Medium. In Allamandola, L. J. and Tielens, A. G. G. M., editors, Interstellar Dust, volume 135 of IAU Symposium, page 431, 1989.

McKee, C. F. and Truelove, J. K. Explosions in the interstellar medium. physrep, 256: 157-172, May 1995. doi: 10.1016/0370-1573(94)00106-D.

Melioli, C. and de Gouveia Dal Pino, E. M. Evolution of the ISM of starburst galaxies: The SN heating efficiency. aap, 424:817-831, September 2004. doi: 10.1051/0004-6361: 20035750.

Melioli, C. and de Gouveia Dal Pino, E. M. Gas Outflows in Seyfert Galaxies: Effects of Star Formation versus AGN Feedback. 812:90, October 2015. doi: 10.1088/0004-637X/ 812/2/90. 
Melioli, C., Brighenti, F., D’Ercole, A., and de Gouveia Dal Pino, E. M. Hydrodynamical simulations of Galactic fountains - I. Evolution of single fountains. MNRAS, 388:573586, August 2008. doi: 10.1111/j.1365-2966.2008.13446.x.

Melioli, C., Brighenti, F., D'Ercole, A., and de Gouveia Dal Pino, E. M. Hydrodynamical simulations of Galactic fountains - II. Evolution of multiple fountains. MNRAS, 399: 1089-1105, November 2009. doi: 10.1111/j.1365-2966.2009.14725.x.

Melioli, C., de Gouveia Dal Pino, E. M., and Geraissate, F. G. Evolution of M82-like starburst winds revisited: 3D radiative cooling hydrodynamical simulations. MNRAS, 430:3235-3248, April 2013. doi: 10.1093/mnras/stt126.

Melioli, C., Brighenti, F., and D'Ercole, A. Galactic fountains and outflows in star-forming dwarf galaxies: interstellar medium expulsion and chemical enrichment. MNRAS, 446: 299-316, January 2015. doi: 10.1093/mnras/stu2008.

Miyamoto, M. and Nagai, R. Three-dimensional models for the distribution of mass in galaxies. pasj, 27:533-543, 1975.

Modjaz, M., Moran, J. M., Kondratko, P. T., and Greenhill, L. J. Probing the Magnetic Field at Subparsec Radii in the Accretion Disk of NGC 4258. Apj, 626:104-119, June 2005. doi: 10.1086/429559.

Morganti, R. The interplay between radio-activity and the ISM in radio galaxies. In Storchi-Bergmann, T., Ho, L. C., and Schmitt, H. R., editors, The Interplay Among Black Holes, Stars and ISM in Galactic Nuclei, volume 222 of IAU Symposium, pages 243-248, November 2004. doi: 10.1017/S1743921304002182.

Morganti, R., Oosterloo, T. A., Tadhunter, C. N., van Moorsel, G., and Emonts, B. The location of the broad $\mathrm{H}$ i absorption in 3C 305: clear evidence for a jet-accelerated neutral outflow. aap, 439:521-526, August 2005a. doi: 10.1051/0004-6361:20053175.

Morganti, R., Tadhunter, C. N., and Oosterloo, T. A. Fast neutral outflows in powerful radio galaxies: a major source of feedback in massive galaxies. aap, 444:L9-L13, December 2005b. doi: 10.1051/0004-6361:200500197.

Morganti, R., Frieswijk, W., Oonk, R. J. B., Oosterloo, T., and Tadhunter, C. Tracing the extreme interplay between radio jets and the ISM in IC 5063. aap, 552:L4, April 2013. doi: 10.1051/0004-6361/201220734.

Morganti, R., Oosterloo, T. A., Oonk, J. B. R., Frieswijk, W., and Tadhunter, C. N. Radio Jets Clearing the Way Through a Galaxy: Watching Feedback in Action in the Seyfert Galaxy IC 5063. In Iono, D., Tatematsu, K., Wootten, A., and Testi, L., editors, 
Revolution in Astronomy with ALMA: The Third Year, volume 499 of Astronomical Society of the Pacific Conference Series, page 125, December 2015.

Murray, N., Quataert, E., and Thompson, T. A. On the Maximum Luminosity of Galaxies and Their Central Black Holes: Feedback from Momentum-driven Winds. Apj, 618: 569-585, January 2005. doi: 10.1086/426067.

Navarro, J. F., Frenk, C. S., and White, S. D. M. The Structure of Cold Dark Matter Halos. Apj, 462:563, May 1996. doi: 10.1086/177173.

Nawaz, M. A., Wagner, A. Y., Bicknell, G. V., Sutherland, R. S., and McNamara, B. R. Jet-intracluster medium interaction in Hydra A - I. Estimates of jet velocity from inner knots. MNRAS, 444:1600-1614, October 2014. doi: 10.1093/mnras/stu1563.

Netzer, Hagai. Revisiting the unified model of active galactic nuclei. Annual Review of Astronomy and Astrophysics, 53(1):365-408, $2015 . \quad$ doi: 10.1146/annurev-astro-082214-122302. URL https://doi.org/10.1146/ annurev-astro-082214-122302.

Novak, G. S., Ostriker, J. P., and Ciotti, L. Feedback from Central Black Holes in Elliptical Galaxies: Two-dimensional Models Compared to One-dimensional Models. Apj, 737: 26, August 2011. doi: 10.1088/0004-637X/737/1/26.

Oosterloo, T. A., Morganti, R., Tzioumis, A., Reynolds, J., King, E., McCulloch, P., and Tsvetanov, Z. A Strong Jet-Cloud Interaction in the Seyfert Galaxy IC 5063: VLBI Observations. aj, 119:2085-2091, May 2000. doi: 10.1086/301358.

Ostriker, J. P., Choi, E., Ciotti, L., Novak, G. S., and Proga, D. Momentum Driving: Which Physical Processes Dominate Active Galactic Nucleus Feedback? MNRAS, 722: 642-652, October 2010. doi: 10.1088/0004-637X/722/1/642.

Pain, R., Hook, I. M., Deustua, S., Gabi, S., Goldhaber, G., Groom, D., Kim, A. G., Kim, M. Y., Lee, J. C., Pennypacker, C. R., Perlmutter, S., Small, I. A., Goobar, A., Ellis, R. S., McMahon, R. G., Glazebrook, K., Boyle, B. J., Bunclark, P. S., Carter, D., and Irwin, M. J. The IA Supernova Rate at Z approximately 0.4. A, 473:356, December 1996. doi: $10.1086 / 178150$.

Parker, E. N. Cosmical magnetic fields: Their origin and their activity. 1979.

Plummer, H. C. On the problem of distribution in globular star clusters. MNRAS, 71: 460-470, March 1911. doi: 10.1093/mnras/71.5.460.

Pounds, K. A. and King, A. R. The shocked outflow in NGC 4051 - momentum-driven feedback, ultrafast outflows and warm absorbers. MNRAS, 433:1369-1377, August 2013. doi: 10.1093/mnras/stt807. 
Pradhan, A. K. and Nahar, S. N. Atomic Astrophysics and Spectroscopy. January 2011.

Pudritz, R. E., Hardcastle, M. J., and Gabuzda, D. C. Magnetic Fields in Astrophysical Jets: From Launch to Termination. ssr, 169:27-72, September 2012. doi: 10.1007/ s11214-012-9895-z.

Raga, A. C., Navarro-González, R., and Villagrán-Muniz, M. A New, 3D Adaptive Grid Code for Astrophysical and Geophysical Gasdynamics. rmxaa, 36:67, April 2000.

Raga, A. C., de Gouveia Dal Pino, E. M., Noriega-Crespo, A., Mininni, P. D., and Velázquez, P. F. Jet/cloud collision, 3D gasdynamic simulations of HH 110. aap, 392: 267-276, September 2002. doi: 10.1051/0004-6361:20020851.

Reis, R. C., Fabian, A. C., Ross, R. R., Miniutti, G., Miller, J. M., and Reynolds, C. A systematic look at the very high and low/hard state of GX339-4: constraining the black hole spin with a new reflection model. MNRAS, 387:1489-1498, July 2008. doi: 10.1111/j.1365-2966.2008.13358.x.

Robitaille, T. P. and Whitney, B. A. The Galactic star formation rate as seen by the Spitzer Space Telescope. Highlights of Astronomy, 15:799-799, November 2010. doi: 10.1017/S1743921310011774.

Rupke, D. S., Veilleux, S., and Sanders, D. B. Keck Absorption-Line Spectroscopy of Galactic Winds in Ultraluminous Infrared Galaxies. Apj, 570:588-609, May 2002. doi: $10.1086 / 339789$.

Rupke, D. S., Veilleux, S., and Sanders, D. B. Outflows in Infrared-Luminous Starbursts at z ; 0.5. I. Sample, Na I D Spectra, and Profile Fitting. Apjs, 160:87-114, September 2005. doi: $10.1086 / 432886$.

Ryan, S. G. and Norton, A. J. Stellar Evolution and Nucleosynthesis. January 2010.

Shapley, A. E. Detailed astrophysical properties of Lyman break galaxies. PhD thesis, CALIFORNIA INSTITUTE OF TECHNOLOGY, 2003.

Sharma, M., Nath, B. B., and Shchekinov, Y. Dust-driven Wind from Disk Galaxies. Apjl, 736:L27, August 2011. doi: 10.1088/2041-8205/736/2/L27.

Simões Lopes, R. D., Storchi-Bergmann, T., de Fátima Saraiva, M., and Martini, P. A Strong Correlation between Circumnuclear Dust and Black Hole Accretion in EarlyType Galaxies. Apj, 655:718-734, February 2007. doi: 10.1086/510064.

Singh, C. B., Mizuno, Y., and de Gouveia Dal Pino, E. M. Spatial Growth of Currentdriven Instability in Relativistic Rotating Jets and the Search for Magnetic Reconnection. Apj, 824:48, June 2016. doi: 10.3847/0004-637X/824/1/48. 
Storchi-Bergmann, T., McGregor, P. J., Riffel, R. A., Simões Lopes, R., Beck, T., and Dopita, M. Feeding versus feedback in NGC4151 probed with Gemini NIFS - I. Excitation. MNRAS, 394:1148-1166, April 2009. doi: 10.1111/j.1365-2966.2009.14388.x.

Storchi-Bergmann, T., Lopes, R. D. S., McGregor, P. J., Riffel, R. A., Beck, T., and Martini, P. Feeding versus feedback in NGC4151 probed with Gemini NIFS - II. Kinematics. MNRAS, 402:819-835, February 2010. doi: 10.1111/j.1365-2966.2009.15962.x.

Strickland, D. K. and Stevens, I. R. Starburst-driven galactic winds - I. Energetics and intrinsic X-ray emission. MNRAS, 314:511-545, May 2000. doi: 10.1046/j.1365-8711. 2000.03391.x.

Tadhunter, C., Morganti, R., Rose, M., Oonk, J. B. R., and Oosterloo, T. Jet acceleration of the fast molecular outflows in the Seyfert galaxy IC 5063. Nat, 511:440-443, July 2014. doi: 10.1038/nature13520.

Tchekhovskoy, A. Launching of Active Galactic Nuclei Jets. In Contopoulos, I., Gabuzda, D., and Kylafis, N., editors, The Formation and Disruption of Black Hole Jets, volume 414 of Astrophysics and Space Science Library, page 45, 2015. doi: 10.1007/978-3-319-10356-3_3.

Thompson, T. A., Quataert, E., and Murray, N. Radiation Pressure-supported Starburst Disks and Active Galactic Nucleus Fueling. Apj, 630:167-185, September 2005. doi: $10.1086 / 431923$.

Tombesi, F., Cappi, M., Reeves, J. N., Palumbo, G. G. C., Yaqoob, T., Braito, V., and Dadina, M. Evidence for ultra-fast outflows in radio-quiet AGNs. I. Detection and statistical incidence of Fe K-shell absorption lines. aap, 521:A57, October 2010. doi: 10.1051/0004-6361/200913440.

Tombesi, F., Cappi, M., Reeves, J. N., Palumbo, G. G. C., Braito, V., and Dadina, M. Evidence for Ultra-fast Outflows in Radio-quiet Active Galactic Nuclei. II. Detailed Photoionization Modeling of Fe K-shell Absorption Lines. Apj, 742:44, November 2011. doi: 10.1088/0004-637X/742/1/44.

Tombesi, F., Cappi, M., Reeves, J. N., Nemmen, R. S., Braito, V., Gaspari, M., and Reynolds, C. S. Unification of X-ray winds in Seyfert galaxies: from ultra-fast outflows to warm absorbers. MNRAS, 430:1102-1117, April 2013. doi: 10.1093/mnras/sts692.

Tombesi, F., Meléndez, M., Veilleux, S., Reeves, J. N., González-Alfonso, E., and Reynolds, C. S. Wind from the black-hole accretion disk driving a molecular outflow in an active galaxy. Nat, 519:436-438, March 2015. doi: 10.1038/nature14261.

Toro, E. Riemann Solvers and Numerical Methods for Fluid Dynamics. 1999. 
Urry, C. M. and Padovani, P. Unified Schemes for Radio-Loud Active Galactic Nuclei. pasp, 107:803, September 1995. doi: 10.1086/133630.

Veilleux, S., Meléndez, M., Sturm, E., Gracia-Carpio, J., Fischer, J., González-Alfonso, E., Contursi, A., Lutz, D., Poglitsch, A., Davies, R., Genzel, R., Tacconi, L., de Jong, J. A., Sternberg, A., Netzer, H., Hailey-Dunsheath, S., Verma, A., Rupke, D. S. N., Maiolino, R., Teng, S. H., and Polisensky, E. Fast Molecular Outflows in Luminous Galaxy Mergers: Evidence for Quasar Feedback from Herschel. Apj, 776:27, October 2013. doi: 10.1088/0004-637X/776/1/27.

Wagner, A. Y., Bicknell, G. V., and Umemura, M. Driving Outflows with Relativistic Jets and the Dependence of Active Galactic Nucleus Feedback Efficiency on Interstellar Medium Inhomogeneity. Apj, 757:136, October 2012. doi: 10.1088/0004-637X/757/2/ 136.

Wagner, A. Y., Umemura, M., and Bicknell, G. V. Ultrafast Outflows: Galaxy-scale Active Galactic Nucleus Feedback. Apjl, 763:L18, January 2013. doi: 10.1088/2041-8205/ $763 / 1 / \mathrm{L} 18$.

Wang, Junfeng, Fabbiano, Giuseppina, Risaliti, Guido, Elvis, Martin, Mundell, Carole G., Dumas, Gaelle, Schinnerer, Eva, and Zezas, Andreas. Extended x-ray emission in the h i cavity of ngc 4151: Galaxy-scale active galactic nucleus feedback? The Astrophysical Journal Letters, 719(2):L208, 2010. URL http://stacks.iop.org/2041-8205/719/ $i=2 / a=L 208$.

Wilman, R. J., Crawford, C. S., and Abraham, R. G. Mapping the gas kinematics and ionization structure of four ultraluminous IRAS galaxies. Mnras, 309:299-324, October 1999. doi: 10.1046/j.1365-8711.1999.02807.x.

Wilson, A. S. and Colbert, E. J. M. The difference between radio-loud and radio-quiet active galaxies. Apj, 438:62-71, January 1995. doi: 10.1086/175054.

Zubovas, K. and King, A. Clearing Out a Galaxy. apjl, 745:L34, February 2012a. doi: 10.1088/2041-8205/745/2/L34.

Zubovas, K. and King, A. R. AGN Winds and the Black-Hole - Galaxy Connection. In Chartas, G., Hamann, F., and Leighly, K. M., editors, AGN Winds in Charleston, volume 460 of Astronomical Society of the Pacific Conference Series, page 235, August 2012b. 